\title{
STATE ESTIMATION FOR DYNAMIC WEIGHING USING KALMAN FILTER
}

\author{
BY \\ SUNETHRA PITAWALA

\begin{abstract}
A thesis
Submitted to the Victoria University of Wellington

In fulfilment of the requirements for the degree of Master of Science
\end{abstract}

Victoria University of Wellington (2019) 


\section{Abstract}

Dynamic weighing has become an essential requirement in a diverse range of industries. Dynamic weighing is different from static weighing in that static weighing involves determining the weight while the product being weighed is stationary whereas dynamic weighing weighs the products while they are moving. Force sensors are commonly used in these weighing systems. In static weighing, the weighed object is placed stationary on the platform and the steady state of the sensor signal is used to assess the weight. However, in dynamic weighing the sensor signal may not reach the steady state during the brief time of weighing, hence the weight is assessed for example, by averaging the tail end of the signal after it has been through a low-pass filter. The resulting mass estimates can be inaccurate for faster heavier items. It is useful to consider better ways of estimating the true weight, in high speed weighing applications.

The proposed method is to employ the 1-D Kalman filter algorithm to estimate the optimal state of the signal. The improved steady state signal is then used in weight estimation. The proposed method has been tested using data collected from a loadcell when different masses pass over the loadcell. The results show a significant improvement in the filtered signal quality which is then used to improve the weight assessment. 


\section{Acknowledgements}

I would like to express my sincere gratitude to Prof. Mark McGuiness for his supervision and support throughout the course of study. He guided me in the right direction while giving me the freedom for this thesis to be my own work.

I would like to extend my sincere thanks to my colleague, Fred Harris for proofreading my thesis and for his useful, detailed comments.

I would like to express my special thanks to Dr. Induka Werellagama for being persistently encouraging and supportive throughout my studies.

I would like to thank Wellington Institute of Technology for providing me with this professional development opportunity and for the financial contribution towards the course fees.

I would also like to say thanks to those who provided answers to my questions via internet forums.

Finally, I would like to thank my family for their support of, and perseverance with, me. 


\section{Contents}

$\begin{array}{lr}\text { Abstract } & \text { i } \\ \text { Acknowledgments } & \text { ii } \\ \text { Contents } & \text { iii }\end{array}$

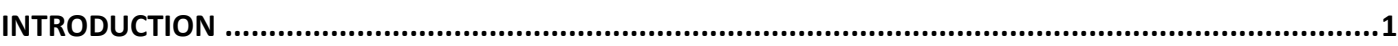

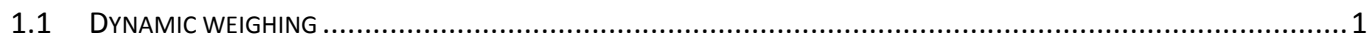

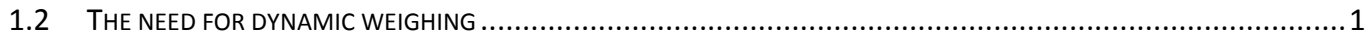

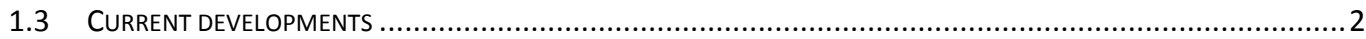

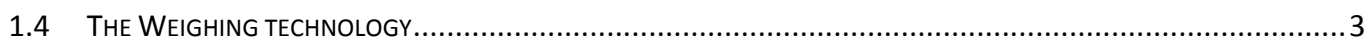

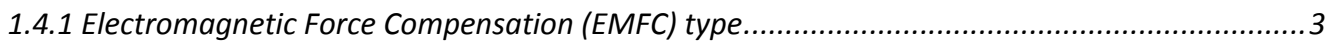

1.4.2 Electro-mechanical Compensation type ..........................................................................

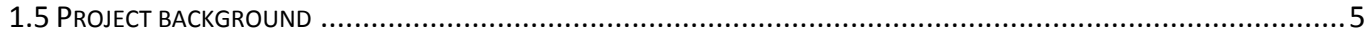

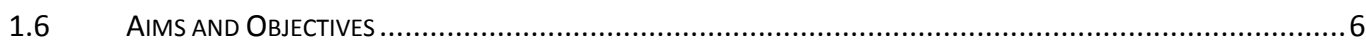

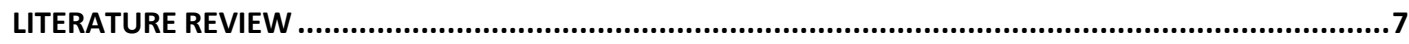

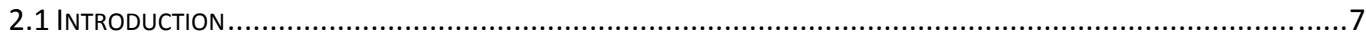

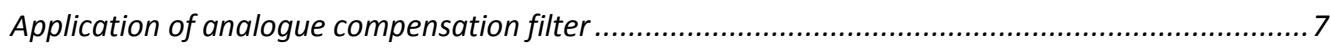

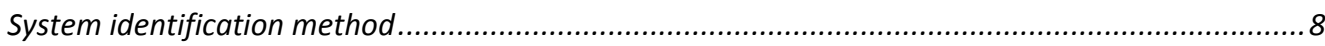

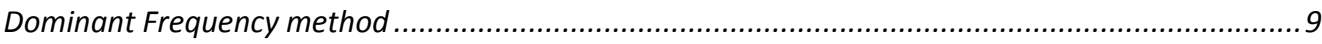

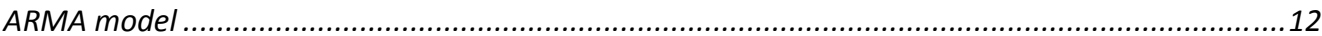

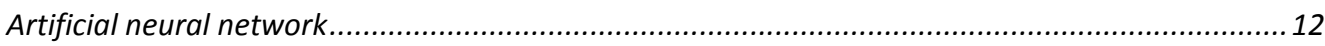

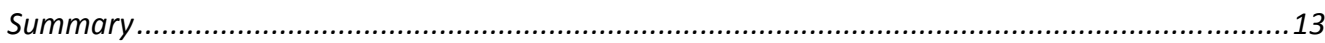

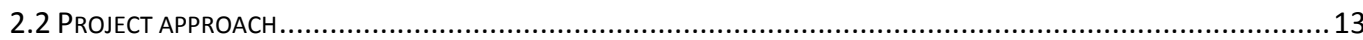

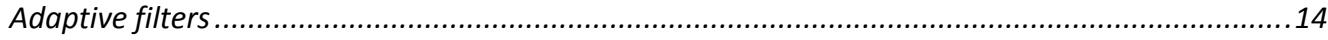

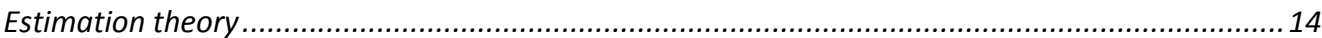

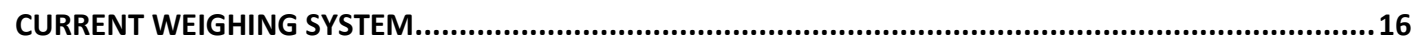

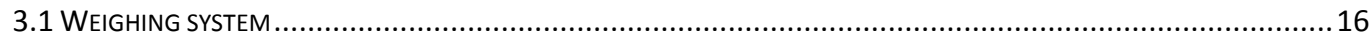

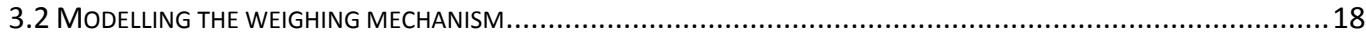

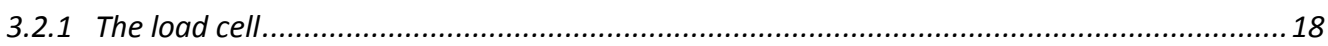

3.2.2 Fifth order Butterworth filter ................................................................................. 23

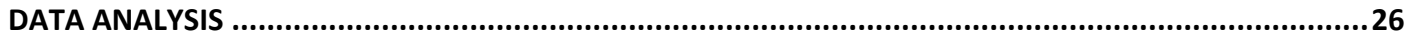

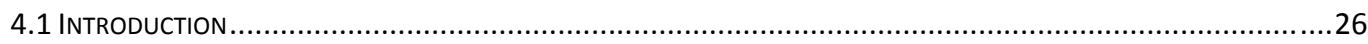

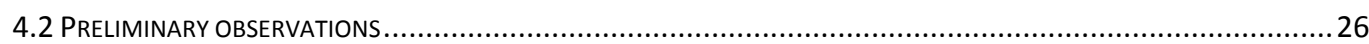

4.2.1. Observations of data as fruits pass over the weigh table ................................................2 27 
4.2.2. Observations of data as empty cups passing over the weighing table .29

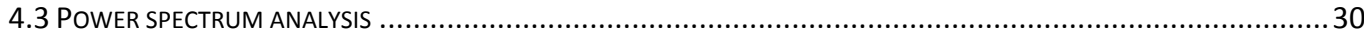

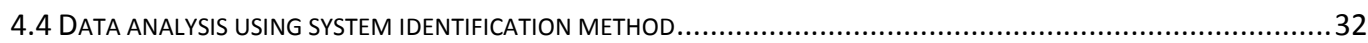

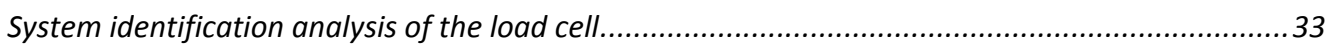

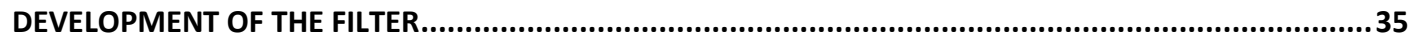

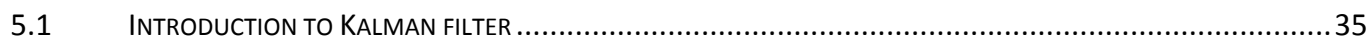

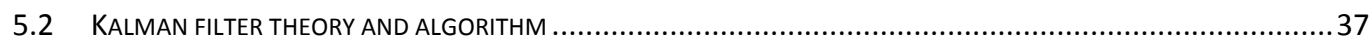

5.2.1 Kalman filter algorithm for a single variable system .................................................... 37

5.2.2 Kalman filter algorithm for multivariate systems...................................................4 41

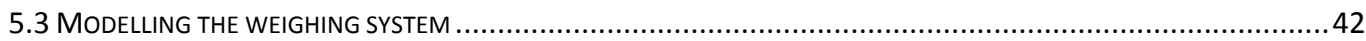

5.3.1 Kalman filter (Using the Second order differential equation as the system model) ...........42

5.3.2 Kalman filter (1 - D, Discrete-time model) ..................................................................44

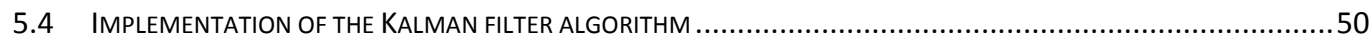

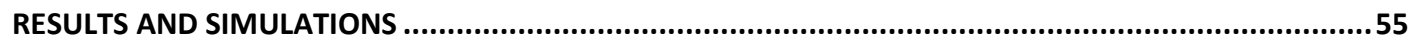

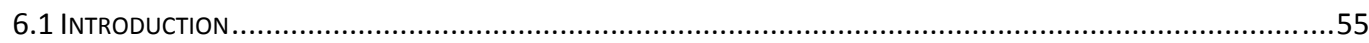

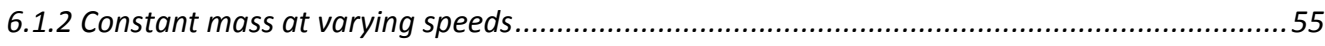

Filtered signal around the plateau (Loaded data) ................................................................5

Filtered signals before arrival at the load cell (Unloaded data)....................................................59

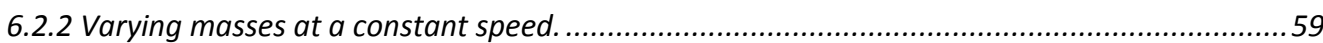

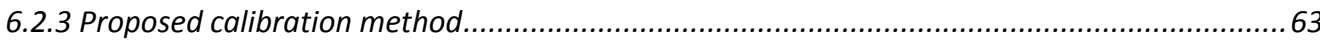

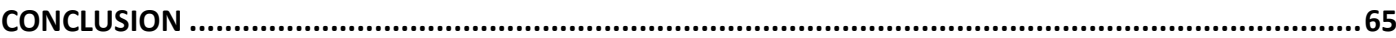

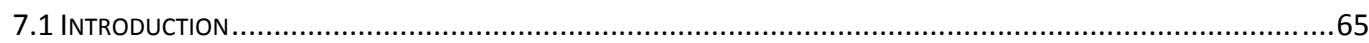

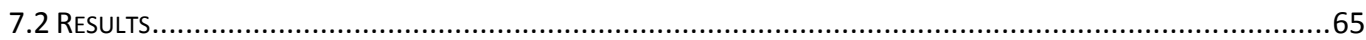

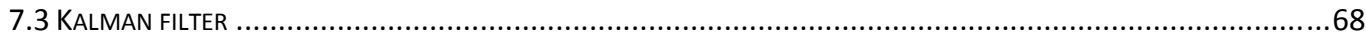

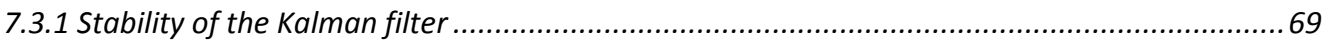

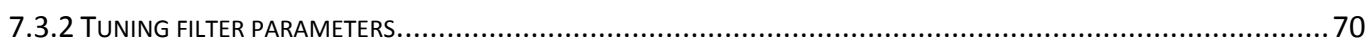

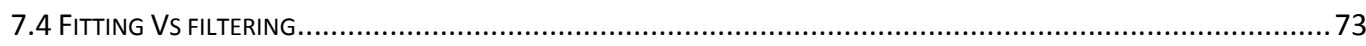

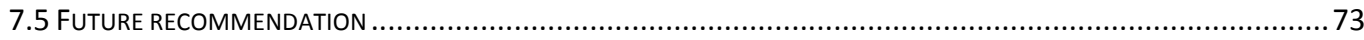

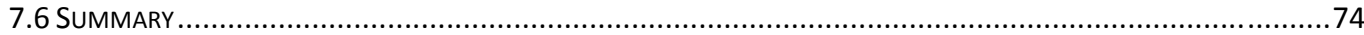

Appendix A Kalman filter MATLAB Code $\quad 75$

$\begin{array}{lll}\text { Appendix B } & \text { Graphs of Kalman filter output and stability } & 76\end{array}$

$\begin{array}{lll}\text { Appendix C } & \text { Poswer spectra analysis } & 81\end{array}$

References $\quad 86$ 


\section{Chapter 1.0 Introduction}

\subsection{Dynamic weighing}

Dynamic weighing refers to the system that weighs products while they are being conveyed over a weighing platform within a production line. Force sensors are commonly used in both of these weighing systems. In static weighing, the weighed object is placed stationary on the platform and the steady state of the sensor signal is used to assess the weight. However, in dynamic weighing the sensor signal may not reach the steady state hence the weight is assessed by averaging the tail end of the signal. It is, therefore, necessary to determine the average value of the signal in the fastest possible time, in high speed weighing applications.

\subsection{The need for dynamic weighing}

Dynamic weighing has become an essential requirement in many industries. It significantly increases the productivity and efficiency that results in reduction in costs and increased profits.

The dynamic weighing systems are developed and available in a diverse range of applications. Weighing in motion (WIM) applications are designed to capture and record the vehicle weights that are useful in bridge and pavement designs, enforcing size and weight restrictions. This information is then used by road transport authorities and administrative departments for planning and legislative processes.

Process weighing systems are employed in recipe formulation and control in manufacturing, food and beverage, bakery, chemical, concrete and pharmaceutical industries.

In the area of mass production, products are weighed while they are in motion. The weight information are then used for other quality control purposes. The products range 
from packaging, bottles, fruit and vegetables and many more. The dynamic weighing systems also known as 'checkweigher' have been a part of production systems for decades.

The dynamic weighing allows automation of the weighing process and it has become an integral part of today's automated production processes.

Precise weight measurements are needed to generate the product labels including the price which is weight dependent. Weighing accuracy is also important as the weight can be translated to the other physical quantities of the product being weighed which in turn is used to decide the quality and the value of the product.

Manufacturers must ensure that the weighed product meets the minimum specification laid down by law or contract. However, the products should not exceed the specifications which results in giving the customers more than they pay for.

As the new developments of technology result in increase of the production rates, there is an increasing need in industry for high speed checkweighing systems with greater accuracy, higher reliability and enhanced control systems. This has been brought about by economic and legal factors.

\subsection{Current developments}

When today's dynamic weighing systems are used, they capture and record the required data within a few seconds and $100 \%$ of manufactured products are checked. The advantages of checkweighers go beyond the speed and 100\% weight assessment. There are stations for quality assurance integrated into the same production facility and they use the information captured by the checkweighers.

There are several different designs of checkweighers depending on their application requirements. The primary components of a checkweigher are an in-feed section, a weighing platform and an out-feed section (The checkweighing guide, 2016).

Items are fed onto the weighing conveyor (Weigh table) from the in-feed conveyor which is typically mounted on a force transducer. A signal processing unit receives a signal from the force sensor and estimates a value of the weight for the product that is being passed over the weigh table. 


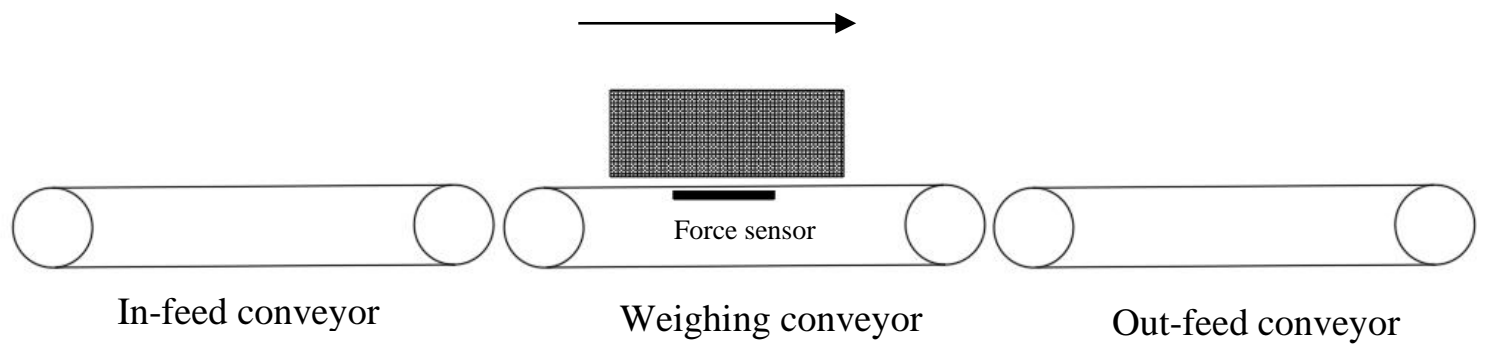

Figure (1.1): Checkweigher arrangement

\subsection{The Weighing technology}

The weighing technology is required to demonstrate different characteristics depending on the application of the checkweigher. The required level of accuracy, throughput, environmental conditions at the installation site and the product specifications are key considerations in selecting a suitable checkweigher for a specific application. Environmental conditions such as temperature, humidity and undesirable vibrations play a considerable role in checkweigher accuracy.

For precision in weighing products, the weighing cell or the weighing sensor technology and the signal process method are the most important components in the checkweigher. There two types of weighing sensor technologies that are commonly available: (laboratory balances, n.d.).

- $\quad$ Electromagnetic Force Compensation (EMFC) type.

- $\quad$ Electro-mechanical Compensation type (Commonly known as strain gauge type).

\subsubsection{Electromagnetic Force Compensation (EMFC) type}

Also known as Magnetic force restoration (MFR), the compensation principle has superior performance characteristics than the conventional systems. (MFR weigh cell features, n.d.) 


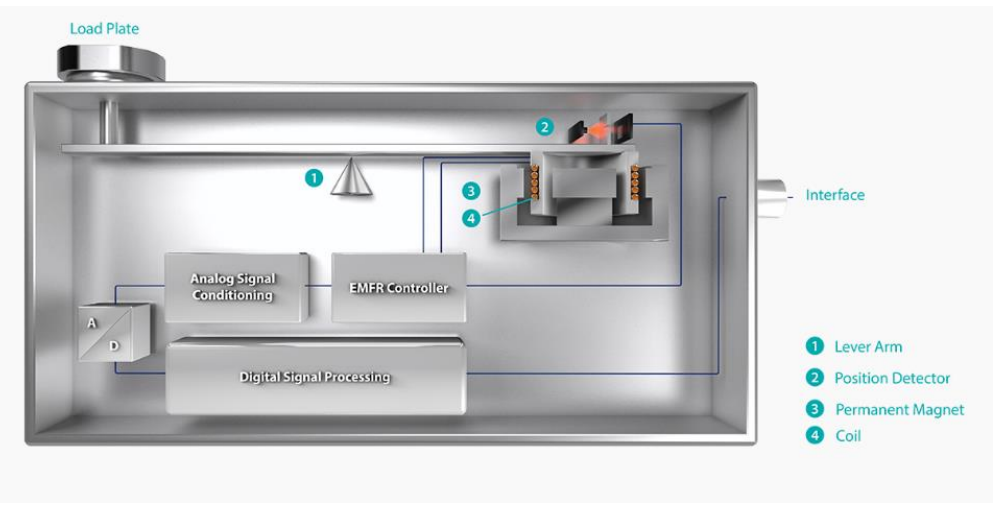

Figure (1.2): A schematic diagram of EMFR type load cell (Weighing principle [online image]. Retrieved $07^{\text {th }}$ June 2019 from https://www.wipotect.com/en/company/weighingprinciple/

The weighing sensor comprises a lever arm, and electromagnetic system, an optical sensor to detect position.

The applied load is counteracted by a restoration force produced by the electromagnet. Any slight movement of the compensation arm initiates a feedback current through a coil and causes the load to be returned to its original position. The current is proportional to the displacement and in turn, proportional to the weight. It is converted into a digital signal and is processed in the microprocessor.

In comparison to the strain gauge load cells, EMFR load cells can be more accurate and can measure at a faster rate (The checkweighing guide, 2016). The self-damping weigh cell achieves a settling time less than $200 \mathrm{~ms}$. This feature of EMFR type loadcells guarantees high precision with shorter measurement times (MFR weigh cell features, n.d).

\subsubsection{Electro-mechanical Compensation type}

Electro-mechanical compensation type load cells (strain gauge load-cells) are the common type of weight transducers in checkweighers and are applicable to a wider range of weights.

Mechanical stress on a strain gauge causes deformation of the gauge. The strain gauge is adhered to the object under test and acts like the material it is adhered to. The strain gauge deforms under applied load and the resistance of the strain gauge varies as the strain gauge material deforms. The change in resistance of the strain gauge is proportional to the deformation and in turn, it is proportional to the applied stress/force. 
The small changes in resistance are turned into more precise values by using the Wheatstone bridge arrangement of the resistors.

The circuit is designed so that the voltmeter reading is zero when there is no load applied on the strain gauges hence there is no change in resistance of the strain gauges.

The voltmeter shows a reading when the load is applied and the resistor circuit is unbalanced (Sharma, 2019).

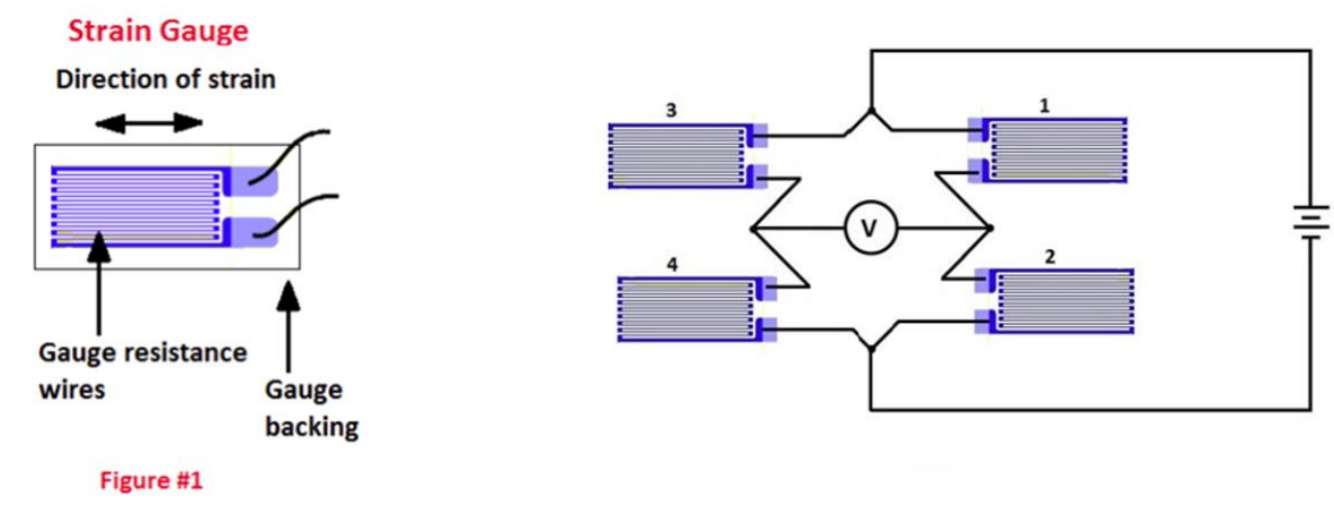

Figure (1.3): (a) Strain gauge (b) Wheatstone bridge circuit (Sharma, 2019)

Strain gauge load cells are often equipped with mechanical overload stops to prevent damage to the load cell if the applied load exceeds the capacity of the load cell.

Some major defects with sensor signals are noise or influence of unwanted signals, parameter drift (change in the item parameters over the time), non-linearity and cross sensitivity, i.e. Change of signal as a result of change in an indirect property such as ambient temperature.

\subsection{Project background}

Compac Sorting Equipment is a New Zealand company that manufactures high speed sorting machines for fruits and vegetables. Fruit is transported in individual plastic carriers attached to a chain, forming a conveyer belt or a lane and each carrier is pulled across a weighing bridge via a floating link attached to a chain. The lanes are of $5 \mathrm{~m}-$ $100 \mathrm{~m}$ length and the typical number of lanes in a machine varies from 6 to 8 .

Each fruit is weighed separately as it passes over the load cells at a rate of $10-12$ fruit per second. Current weighing system yields a repeatability standard deviation better than 0.5 with $200 \mathrm{~g}$ test weight at 10 fruit per second. Weighing accuracy deteriorates with fruit heavier than $250 \mathrm{~g}$ and at speeds higher than 10 fruit per second. 
Compac requires an improved signal processing method for determining the true weight of fruits. As a part of the expected improvement, the settling period of the signal required to be reduced so that the signal reaches the steady state prior to the data sampled for weight assessment (Compac sorting equipment, personal communication, 2005).

Time series data of the load cell readings of fruits passing over the load cell are provided from number of fruits at three different speeds.

Compac have indicated the possibilities of using position sensors to select a set of data, data showing a response to the weight of the fruit.

\subsection{Aims and Objectives}

The overall aim of the project is to develop a method to improve the weighing accuracy of fruit with fruit heavier than $250 \mathrm{~g}$ and at speeds higher than 10 fruits per second. The dynamic behaviour of the current weighing system will be studied in detail with the use of a mathematical model. Time series data provided will be used to test the system.

The focus of this research is to develop a better signal processing method that improves the accuracy of measurements by,

(i) Obtaining a smoother steady state signal by removing the influence of unwanted signals (noise).

(ii) Improve the filter response time and thereby reduce setting time of the signal. 


\section{Chapter 2.0 Literature Review}

\subsection{Introduction}

Many methods have been proposed and investigated to improve the weight estimation in high speed weighing in the past few decades. The rest of this chapter discusses the various solutions proposed in previous years.

\section{Application of analogue compensation filter}

Jafaripanah, Al-Hashmi \& White (2005) proposed an analogue, compensation filter as a possible method to improve the accuracy of mass ascertainment in dynamic weighing systems.

The sensor (loadcell) dynamics was modelled in the form of a second order system,

$$
(M+m) \frac{d^{2} x(t)}{d t^{2}}+c \frac{d x(t)}{d t}+k x(t)=F(t) .
$$

where $\mathrm{M}-$ mass of the carrier

$\mathrm{m}$ - mass of the fruit

$\mathrm{x}$ - deflection of the loadcell

$\mathrm{c}$ - damping coefficient

$\mathrm{k}$ - spring constant

F - Force applied on the load cell by the fruit

The transfer function of the load cell,

$$
G(s)=\frac{X(s)}{F(s)}=\frac{\frac{1}{(M+m)}}{s^{2}+\frac{c}{(M+m)} s+\frac{k}{(M+m)}} .
$$

where $X(s)$ and $F(s)$ are the Laplace transforms of $\mathrm{x}(\mathrm{t})$ and $\mathrm{F}(\mathrm{t})$.

The pair of complex conjugate poles of the system has been written in the form $a \pm i b$ and both $a$ and $b$ are functions of unknown mass that is to be estimated. 


$$
a=-\frac{c}{2(M+m)}, \quad b=\sqrt{\frac{k}{(M+m)}-\frac{c^{2}}{(M+m)^{2}}} .
$$

An analogue compensation filter was designed using the pole locations of the system, $a \pm i b$, as zeros of the filter. Two additional poles have been added to the filter and the location of the additional poles has been selected using the trial and error method. The zeros of the filter cancel the poles of the sensor.

The transfer function of the compensation filter is a bi-quadratic function, with $a \pm i b$ as locations of the zeros and poles at $d \pm i e$ where $d$ and $e$ specify the location of the additional poles.

The transfer function of the compensating filter is,

$$
H(s)=A \frac{(s-a-i b)(s-a+i b)}{(s-d-i e)(s-d+i e)} .
$$

An analogue, state variable low pass filter with a biquadratic structure was used as the compensating filter. In order to make the analogue filter adaptive, a voltage-controlled resistor has been used. The voltage-controlled resistor models the mass to be estimated.

The filter produces an output voltage that is proportional to the mass to be estimated which also changes the value of the resistance in proportion to the mass. Using the filter transfer function, the locations of the corresponding pole locations can be determined which in turn can be used to calculate the mass of the fruit.

The simulated results show that the state variable, biquadratic filter provides an accurate and flexible adaptive compensation filter.

However, in the simulation results presented the poles had been selected by trial and error so that the output of the filter quickly reaches the steady state with minimum oscillations. The speed of the system is not stated.

\section{System identification method}

Shu, (1993) proposed a system identification based method for mass estimation for checkweighers. The checkweigher is modelled as the spring-mass-damper system and the input to the checkweigher is a step function with a magnitude of $\mathrm{m} g$. The discrete model for the spring - mass- damper system is derived from the continuous system equation that is similar to the system equation employed by Jafaripanah, Al-Hashmi \& White (2005) (Equation (2.1).) 


$$
(M+m) \frac{d^{2} x(t)}{d t^{2}}+c \frac{d x(t)}{d t}+k x(t)=m g u(t)
$$

A Laplace transformation of the second order equation has been used to develop the $\mathrm{z}$ transform of the system equation. (Equation (2.3)).

$$
X(z)=m g U(z) \frac{1}{k} \frac{x(0)+b_{1}^{\prime} z^{-1}+b_{2}^{\prime} z^{-2}}{1+a_{1} z^{-1}+a_{2} z^{-2}}
$$

where $z$ is a complex number and $b_{1}^{\prime}$ and $b_{2}^{\prime}$ are coefficients that contain the mass to be estimated.

With the use of the end value theorem ${ }^{1}$, it is shown that the values at the infinity are proportional to the mass of the fruit. Thus, the latter part of the signal can be used to determine the mass of the fruit.

The unknown parameters of the model are estimated by fitting the model to the measured signal and the optimal fit is achieved using the recursive least square algorithm. The estimated model parameters are then used to estimate the mass being weighed.

Shu, (1993) also shows that the algorithm is independent of the initial conditions and thereby the method is independent of the initial conditions.

In order to obtain better results, a low pass filter is cascaded with the algorithm to improve the data quality.

\section{Dominant Frequency method}

McGuiness, Jenkins \& Senaratne (2005) investigated the use of the dominant frequency of the system to estimate the mass. Solving the mathematical model of the loadcell (equation 2.1) gives the natural frequency of oscillations for simple harmonic motion,

$$
\omega=\frac{k}{M+m} .
$$

It can be seen that the natural frequency $\omega$ decreases as the mass increases. The tests had been carried out to obtain the dominant frequency of each data set which can be used to estimate the mass on the loadcell with the use of the equation (2.4).

Power spectra analysis was used to obtain the dominant frequency of the system. The results of numbers of tests infer that a dominant frequency of $120 \mathrm{~Hz}$ was almost independent of the mass. It had been also noted that there were two or three dominant frequencies in each

\footnotetext{
${ }^{1}$ End value Theorem: If $X(s)$ is multiplied by $s$ and take limit as $s \longrightarrow 0$, the result will be the final value of $x(t)$, i.e. $x(\infty)$, (Bateson, 2002).
} 
spectrum. The higher frequency of $120 \mathrm{~Hz}$ had been found to be out of phase between the two loadcells and to be associated with the rocking motion of the fruit. The lower frequencies that are in phase between the loadcell had been found to be associated with the bouncing motion of the fruit.

Shown in figure (2.1) are the power spectra obtained for $200 \mathrm{~g}$ moving at $0.5 \mathrm{~m} / \mathrm{s}$ and 1.5 $\mathrm{m} / \mathrm{s}$, as a part of this thesis. In addition to the dominant frequency of $120 \mathrm{~Hz}$ which is present in all the power spectra, a peak is observed around $70 \mathrm{~Hz}$ in $200 \mathrm{~g}$ moving at $0.5 \mathrm{~m} / \mathrm{s}$ and the peak in $200 \mathrm{~g}$ at $1.5 \mathrm{~m} / \mathrm{s}$ is around $20 \mathrm{~Hz}$. The level and frequency components change with the speed implying that the conveyor belt speed contributed to the peaks of the power spectra which need to be considered in estimating the mass using the dominant frequency method. Further analysis of power spectra is given in 'Data analysis' in Chapter 4.0.
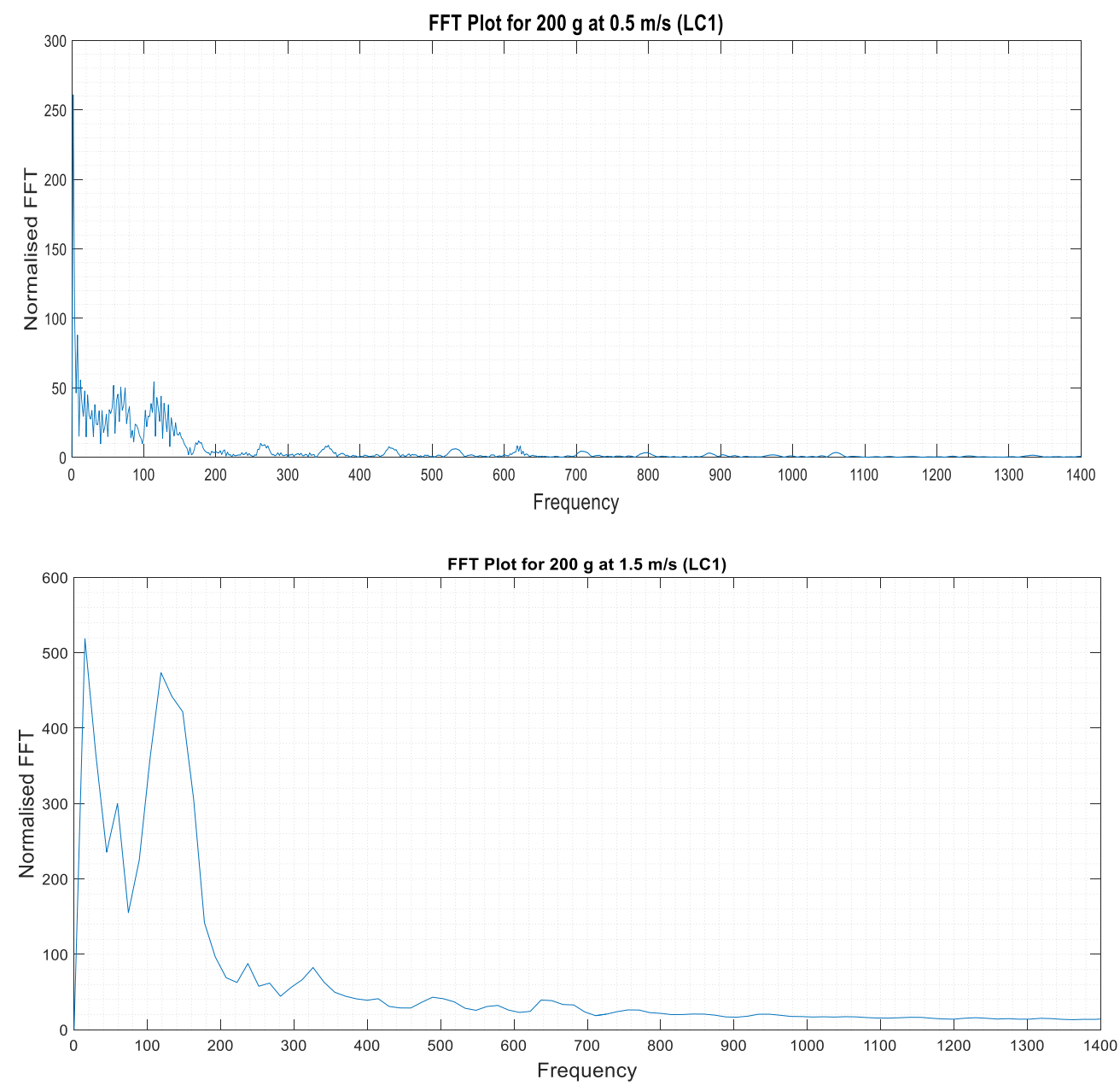

Figure (2.1): Power Spectra for $200 \mathrm{~g}$ at $0.5 \mathrm{~m} / \mathrm{s}$ and $1.5 \mathrm{~m} / \mathrm{s}$ 
The requirement of this project is to obtain weight estimates of high accuracy, in the range of $\pm 1 \mathrm{~g}$.

Given that the elastic property of a harmonic oscillator is represented by $k$ and the mass is $m_{1}$, the natural frequency of the harmonic oscillator is,

$$
\omega_{1}=\sqrt{\frac{k}{m_{1}}}
$$

and if the mass is increased to $m_{2}$, the new natural frequency is,

$$
\omega_{2}=\sqrt{\frac{k}{m_{2}}}
$$

Then,

$$
\left(\omega_{1}-\omega_{2}\right)^{2}=\left(\sqrt{\frac{k}{m_{1}}}-\sqrt{\frac{k}{m_{2}}}\right)^{2}
$$

For very small changes of mass (i.e. from $0.2 \mathrm{~kg}$ to $0.3 \mathrm{~kg}$ ) $m_{1} \approx m_{2}$,

$$
\left(\sqrt{\frac{k}{m_{1}}}-\sqrt{\frac{k}{m_{2}}}\right)^{2}=\left(\omega_{1}-\omega_{2}\right)^{2} \approx 0
$$

The change in frequency corresponds to the small change in mass is very small. Observed in the power spectra, filtering frequencies to a higher accuracy in the range of $\pm 1 \mathrm{~Hz}$ cannot be achieved in practice.

McGuiness et al. (2005) also propose a new system model based on undamped free vibration of a cantilever beam shown in equation (2.5).

$$
\rho A \frac{d^{2} y}{d t^{2}}+E I \frac{d^{4} y}{d x^{4}}=0
$$

It can be seen that the model developed (equation (2.5)) has similar characteristics to the previous mathematical models developed (e.g. equation (2.1)). Both system models are second order differential equations in the time domain. In equation (2.5), the coefficient of the acceleration term, $\rho A$, represents the mass properties of the beam (the mass of the loadcell per unit length) and the coefficient of the displacement term, EI, represents the 
elastic properties (flexural stiffness ${ }^{2}$ ) of the beam. The damping coefficient and the input function have been set to zero for undamped, free vibration considered in the proposed system model.

This model provides a better representation of the vibration properties of the loadcell if suitable damping properties are included.

\section{ARMA model}

Calpe, J. et al. (2002) propose a two stage filtering technique. An adaptive filter is used to remove the mechanical vibration in the system in the first stage and the resulting signal is deconvolved using a second order $\mathrm{ARMA}^{3}$ model. The mass of the fruit was estimated by averaging the filtered signal.

In the first stage of filtering, the filter value is set to a constant, the error between the constant and the signal value is minimised using the least mean square algorithm.

The resulting signal represents the dynamics of the load cell under the applied load. The coefficients of the ARMA model were determined using an iterative, robust quadratic prediction error method. The deconvolution of the original signal based on the ARMA model has resulted in a relatively plain signal which was used to estimate the weighing using a calibration method.

The weight obtained using this method has been in the range of $\pm 1 \mathrm{~g}$ at the speed of 16 fruits per second.

\section{Artificial neural network}

Yasin \& White (1999) proposes a technique based on artificial neural network. The load sensor is a tri-beam loadcell which consists of three cantilever beams coupled together which was modeled as a non-linear system. The input to the non-linear system is the unknown load and the output is the output voltage signal.

Simulated response of $100 \mathrm{~g}$ and $600 \mathrm{~g}$ was used to show the final value can be anticipated from three successive extreme points. These three points were chosen as the features of the output signal.

\footnotetext{
${ }^{2}$ Measure of the ability to bend or deform.

${ }^{3}$ ARMA - Mathematical models that combine the effect of weighted sum past data and weighted sum of moving average of past data.
} 
The neural network was trained using a selected set of masses. A number of test results were presented for $150 \mathrm{~g}, 350 \mathrm{~g}, 550 \mathrm{~g}, 750 \mathrm{~g}$ and $950 \mathrm{~g}$ which are different from the masses used for training the neural network. A random error of $\pm 1.5 \%$ with mean close to zero has been observed.

The results were then verified by means of practical experiments. A maximum error of $\pm 1.5 \%$ has been recorded repeatedly when tested with different conditions: by changing the number of layers and by changing the number of neurones in each layer.

The authors have presented a new method for dynamic weighing. However, the information about the speeds used, was not presented.

\section{Summary}

Many methods that can be used to improve the weighing accuracy are found in literature. Some methods are model-based and some use a model-free approach.

In the model-based approach, the strain gauge load cell has been modelled as a second order differential equation with respect to the time variable.

The methods proposed can be classified as follows:

- $\quad$ Model parameter estimation method.

- $\quad$ Neural network method.

- $\quad$ Frequency compensation method.

Advantages and disadvantages of each method were discussed under each method.

\subsection{Project approach}

One of the key mathematical operations in digital filtering is convolution. The convolution of the two signals are defined as,

$x(n) \circledast h(n)=\sum_{k=-\infty}^{\infty} h(k) x(n-k)$; where $n=0,1, . .(M-1)$ and $M=N 1+N 2-1$. $N 1$ and $N 1$ are the lengths of sequences $x(n)$ and $h(n)$ respectively. 
The output of the digital filter, $y(n)$ is obtained by convolution of the input signal $x(n)$ and the impulse response ${ }^{4}$ of the filter (filter Kernel) $h(n)$ in the time domain (Efeachor \& Jervis, 1995), i.e.

$$
y(n)=x(n) * h(n)
$$

The problem involves finding the steady state of the load cell output signal that can be used to estimate the mass of the fruits accurately, through calibration. The existing Butterworth filter satisfactorily removes noise in the unfiltered signal but the filtered signal sometimes takes a considerable time to settle sufficiently before an accurate estimate for the mass is achievable.

It is reasonable to draw the following inferences from data analysis and preliminary research.

- The filter needs to be an adaptive filter to handle the varying noise characteristics introduced to the signal at various speeds and at various masses of the fruits.

- Observed in power spectra of the unfiltered signals there is no clear evidence that the noise and information signals are not overlapping. In cases where the noise and the information signals are overlapped in spectrum where frequency based methods are not effective, information is retrieved through estimations, smoothing or prediction (Khan, 2011).

\section{Adaptive filters}

An adaptive filter has adjustable filter parameters. The output signal of the filter is compared to a desired response signal by subtracting the two samples at a given time. The difference is known as the error signal which is used as the input to a mathematical process which alters or adapts filter parameter in a well-defined manner.

Adaptive filters can be realised using software or hardware techniques. The focus of this project is to propose a mathematical form of adaptive filter.

\section{Estimation theory}

Estimation theory is a branch of statistics which also used in signal processing. Estimating a set of parameters that describes a state of a system from noisy measurements along with a model for the relationship between the measurement and the underlying state. The dynamics

\footnotetext{
${ }^{4}$ The response of a system when the input is an impulse ("a signal consists of a single sample at time 0 having amplitude 1, preceded and followed by zeros" (Smith, 2007)).
} 
of the states are represented by a mathematical model. The uncertainties of the system model and the uncertainties in measurements are incorporated into the estimation process by using the Bayesian Rule of conditional probability to obtain an optimal estimate of the state.

Research was carried out in the area of state estimation based filtering techniques.

Predictive filters are a family of estimation techniques and are essential tools in fields such as robotics, computer graphics and economics. Based on the estimation techniques, the predictive filters assimilate uncertain prediction from the dynamics of the system and noise corrupted observations to estimate the optimal state of a system.

The Kalman filter is of the simplest form of the predictive filters (Goldstein, 2004). In his famous publication in 1960, Rudolf Kalman proposed the Kalman filter algorithm as an improvement to the Wiener filter (An optimal state estimating technique) which is based on convolution of the input signal with the impulse response of the Wiener filter (Kalman, 1960). Explaining the limitations of the Wiener filter he states that "Numerical determination of the optimal impulse response is often quite involved and poorly suited to machine computation. The situation gets rapidly worse with increasing complexity of the problem." The Kalman filter estimates the optimal state of a given set of data (Goldstein, 2004), hence the filter is adaptive to the given data.

Development of the system model and the use of the Kalman filter algorithm are explained in Chapter 5.0. 


\section{Chapter 3.0}

\section{Current Weighing system}

\subsection{Weighing system}

The weighing system consists of an in-feed conveyor, weighing station and out-feed conveyor. Fruit are transported in individual carriers and the chain driven carriers travel over the weighing station which is equipped with a dual load cell system, i.e. platform mounted on two strain gauge load cells as depicted in Figure (3.1).

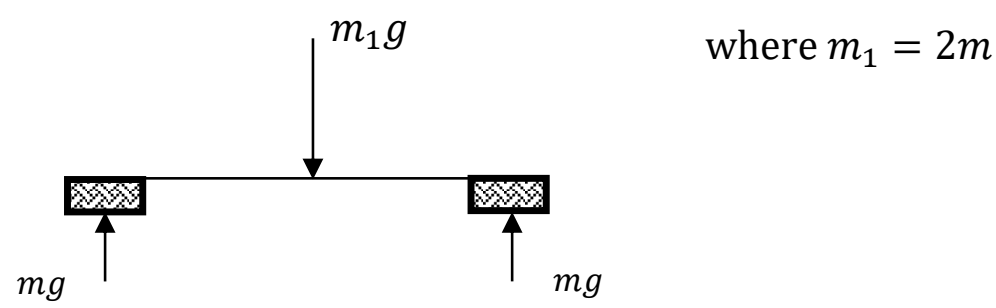

Figure (3.1): Schematic Diagram of the dual load cell arrangement

where; $\quad m_{1} g-$ weight of the fruit

$m g$ - weight applied on the one loadcell

The load cells are mounted on either side of the weighing station and are subjected to half the weight of the fruit being weighed. The load cells are cantilevered to allow deflection. Weighing stations are isolated from the rest of the machine to eliminate the effect of vibrations propagating through other machine components.

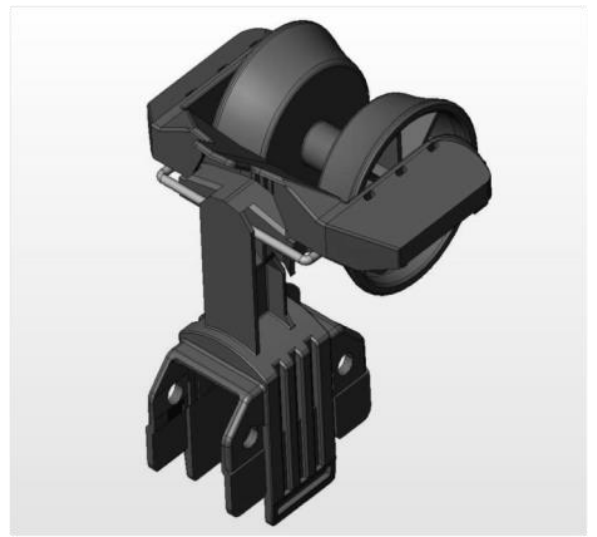

Figure (3.2): Fruit carriers 


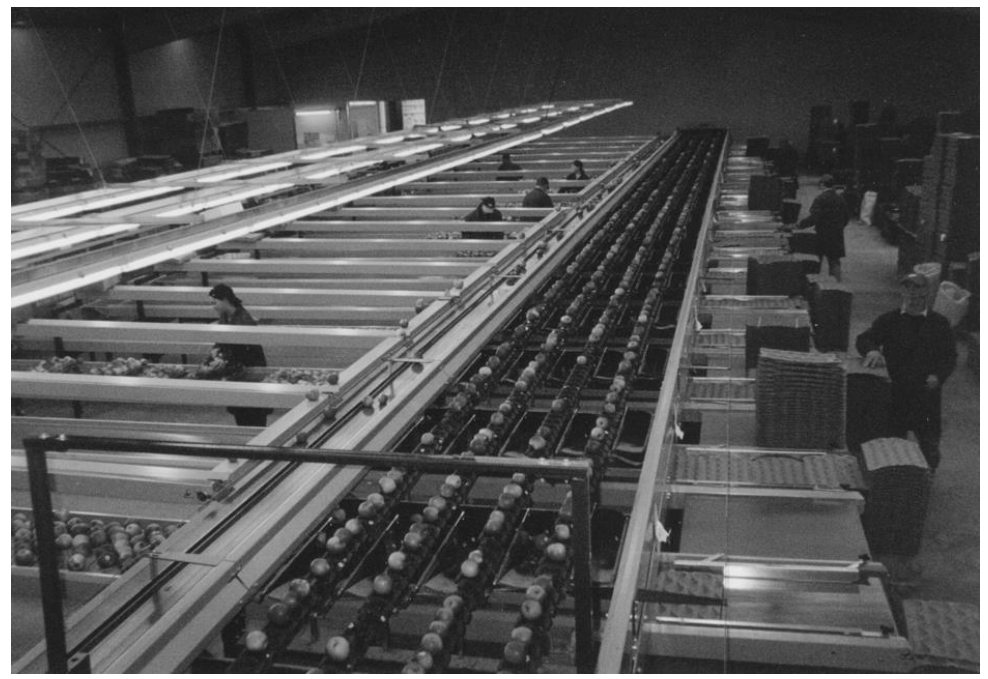

Figure (3.3): A four lane fruit sorting machine

The dual load cell arrangement provides stability to the weighing mechanism. It reduces the possible effects of rocking motion of fruit on the actual weight measured. The load cells used are $6 \mathrm{~kg}$, single point load cells. Single point load cells accurately measure weights regardless of the position on the scale the load is applied. They are strain gauge type load cells which measure the change in resistance in response to the strain applied to the load cell. The small changes in resistances are turned into more precise values by using the Wheatstone bridge arrangement of the resistors. The output voltage of the strain gauge circuit (load cell) is proportional to the strain in the load cell which is proportional to the applied load.

The output voltage of the load cell is amplified and filtered by a fifth order, analogue Butterworth filter and sampled at a rate around $4 \mathrm{kHz}$ by a 12 bit 'analogue to digital converter' (ADC).

The readings in the filtered signal are averaged over a set 'weighing window'. This window is a given percentage of the full cycle. It is also given that the current system achieves a repeatability standard deviation of less than 0.5 when $200 \mathrm{~g}$ moving at 10 fruit per second.

The data provided by Compac include filtered and unfiltered data from the load cells. Figure (4.1) in Chapter 4.0 shows an example of the response of the load cell, filtered and unfiltered data observed during one weighing cycle.

There are several types of load cells: Shear beam, bending beam, compression types are to name few. The dynamic behaviour of these load cells can be represented as spring-massdamper systems. 


\subsection{Modelling the weighing mechanism}

The mathematical model is a set of mathematical expressions that represent the dynamics of the system using laws of physics. This mathematical model permits prediction and study of the system transients and steady state performance.

Primarily, the weighing mechanism is a cascaded system of the 'strain gauge load cell' and the 'fifth-order, analogue Butterworth filter'.

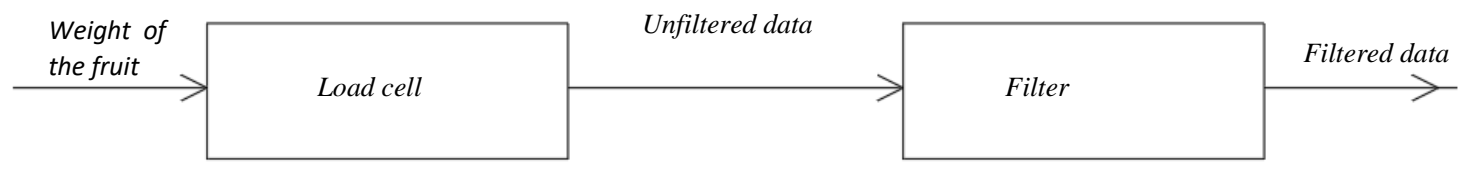

Figure (3.4): The block diagram showing the data flow through the weighing system

\subsubsection{The load cell}

The load cells are cantilevered to allow deflection. As the fruit and the carrier apply the weight force on the load cell, it deflects and set in to oscillations.

Two mathematical models have been used to model the weighing system in previous studies. Jafaripanah et al. (2005) model the system as a spring- mass- damper system as shown in figure (3.4). The mathematical model used is of the form,

$$
(M+m) \ddot{x}+c \dot{x}+k x=m g U(t)
$$

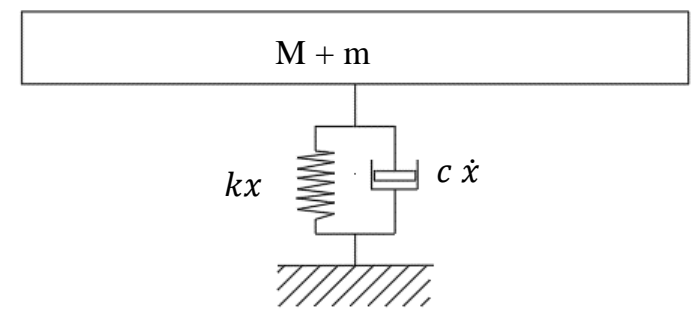

Figure (3.5): Spring- mass- damper system

$M$ - component of the mass of the fruit carrier on one loadcell

$m$-component of the mass of the fruit on one loadcell

$c$-damping coefficient of the spring - mass-damper system

$k$ - spring constant of the spring - mass-damper system

$x$ - deflection of the load cell under the applied load at time $t$

$U-$ unit step function 
McGuiness et al. (2005) model the load cell as a cantilever beam and the mathematical model used represents the free vibration of a cantilever beam (equation 2.4). Both models share similar characteristics as explained in Chapter 2.3.

Laplace transform ${ }^{5}$ of a continuous time domain function $f(t)$ is denoted $L\{f(t)\}$ or by an alternative notation $F(s)$ and defined as,

$$
L\{f(t)\}=F(s)=\int_{0}^{\infty} e^{-s t} f(t) d t
$$

The transfer function was obtained by taking the Laplace transform of equation (3.1),

$$
(M+m)\left(X(s) s^{2}-s x(0)-\dot{x}(0)\right)+c(s X(s)-x(0))+k X(s)=m g L\{U(t)\}
$$

where $X(s)$ is the Laplace transform of $x(t)$.

$$
\begin{gathered}
\text { Using, } L\{U(t)\}=\frac{1}{s} \\
X(s)\left((M+m) s^{2}+c s+k\right)=m g\left(\frac{1}{s}\right)+(M+m)(s x(0)+\dot{x}(0))+c x(0)
\end{gathered}
$$

The transfer function of the system,

$$
H(s)=X(s)=\frac{m g}{s\left((M+m) s^{2}+c s+k\right)}+\frac{(M+m)(s x(0)+\dot{x}(0))+c x(0)}{\left((M+m) s^{2}+c s+k\right)}
$$

Using the initial value theorem: $\lim _{t \rightarrow 0} x(t)=\lim _{s \rightarrow \infty} s X(s)$, (Bateson, 1999, p. 140) the value of the transfer function:

$$
\lim _{s \rightarrow \infty} s X(s)=\lim _{s \rightarrow \infty} s\left[\frac{m g}{s\left((M+m) s^{2}+c s+k\right)}+\frac{(M+m)(s x(0)+\dot{x}(0))+c x(0)}{\left((M+m) s^{2}+c s+k\right)}\right]=0
$$

Using the final value theorem, $\lim _{t \rightarrow \infty} x(t)=\lim _{s \rightarrow 0} s X(s)$, (Bateson, 1999, p. 140) the value of the transfer function:

\footnotetext{
${ }^{5}$ Laplace transform converts a time domain signal into a frequency domain where the magnitude and the phase angle of the signal is determined. The transform also converts differential equations into algebraic equations. (Bateson, 1999, p. 120).
} 


$$
\begin{gathered}
\lim _{s \rightarrow 0} s X(s)=\lim _{s \rightarrow 0} s\left[\frac{m g}{s\left((M+m) s^{2}+c s+k\right)}+\frac{(M+m)(s x(0)+\dot{x}(0))+c x(0)}{\left((M+m) s^{2}+c s+k\right)}\right] \\
\lim _{s \rightarrow 0} s X(s)=\frac{m g}{k}
\end{gathered}
$$

These limits describe the system response to a step function when $t=0$ (the initial value) and as $t \rightarrow \infty$ (the final value). When $t \rightarrow \infty$, the system reaches the steady state therefore the final value $(\mathrm{mg} / \mathrm{k})$ is also the steady state value of the system response.

Assuming that the initial velocity, $\dot{x}(0)=0$ and the initial displacement, $x(0)=0$, the equation (3.2) can be re-written as,

$$
\begin{aligned}
H_{L}(s)= & \frac{\left(\frac{m g}{k}\right) \omega_{n}^{2}}{s\left(s^{2}+2 \xi \omega_{n} s+\omega_{n}^{2}\right)} \\
& \text { where, } \omega_{n}=\sqrt{\frac{k}{M+m}} \text { and } \xi=\frac{c \omega_{n}}{2 k}
\end{aligned}
$$

For an under-damped system, $\xi<1$, the system has three poles and no zeros. One of the poles is located at the origin, and the other two poles form a complex conjugate pair, $-\xi \omega_{n} \pm i \omega_{n} \sqrt{1-\xi^{2}}$

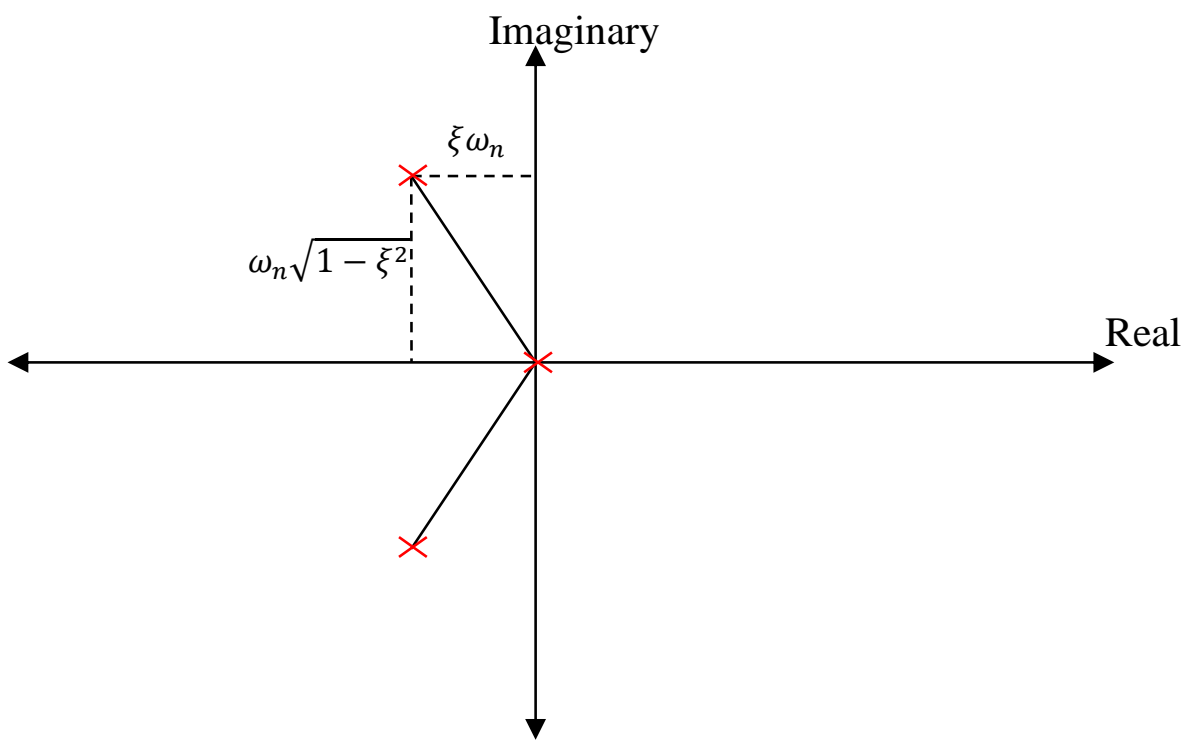

Figure (3.6): Pole-zero plot of the step response of the second order function 
The pole at the origin defines a constant component of the system. The complex conjugate pair in the left half of the s-plane forms an oscillatory component of the system response in time domain indicating 'damped sinusoidal response' of the system.

The time constant, $\tau$ is given by $\frac{1}{\xi \omega_{n}}$ and the magnitude of the product $\xi \omega_{n}$ determines the rate of exponential decay of the response function.

The settling time is the time that the error between the system response and the steady state value reach and stay within a given percentage of the steady state value. The common values of these percentages are $2 \%$ and $5 \%$. The setting time based on the $2 \%$ criterion is approximately $\frac{4}{\xi \omega_{\mathrm{n}}}$ seconds. Hence the response function is significant only for approximately $\frac{4}{\xi \omega_{n}}$ seconds.

The settling time is $\frac{3}{\xi \omega_{n}}$ if the $5 \%$ criterion is considered.

The time domain behaviour of the step response is obtained using the partial fraction and inverse Laplace transform of equation (3.4).

$$
x(t)=\frac{m g}{k}-\frac{m g}{k} \frac{\left(2 \xi \omega_{n}+1\right)}{\omega \sqrt{1-\xi^{2}}} e^{-\xi \omega t \sqrt{1-\xi^{2}}}
$$

The expected time domain behaviour of the load cell output represented by the second order differential equation is shown in figure (3.7). The actual voltage output of the unfiltered signal for $200 \mathrm{~g}$ at $0.5 \mathrm{~m} / \mathrm{s}$ and $573.1 \mathrm{~g}$ at $0.5 \mathrm{~m} / \mathrm{s} 1.5 \mathrm{~m} / \mathrm{s}$ are shown in figure (3.8 (a)) and (3.8 (b)).

The model response and actual data plots shows that the values suddenly increase to a new value (a constant component of the values) where it settles as the time progresses. The model settles faster after two dominant peaks. The unfiltered data for $200 \mathrm{~g}$ at $0.5 \mathrm{~m} / \mathrm{s}$, shows a similar characteristics to that of model response, however, the presence of extra oscillations makes the graph fluctuate around a constant value.

$573.1 \mathrm{~g}$ at $0.5 \mathrm{~m} / \mathrm{s}$, demonstrates the constant component. The signal is highly oscillatory during the entire signal time.

In comparison, the actual data plots exhibit some similarities to the model behaviour with some deviations. 
The second order system equation is developed based on Newton's second Law therefore it precisely represents the system dynamics in ideal conditions. Deviation from this expected pattern implies the presence of other elements such as system disturbances, interference and other vibrations noise in the system.

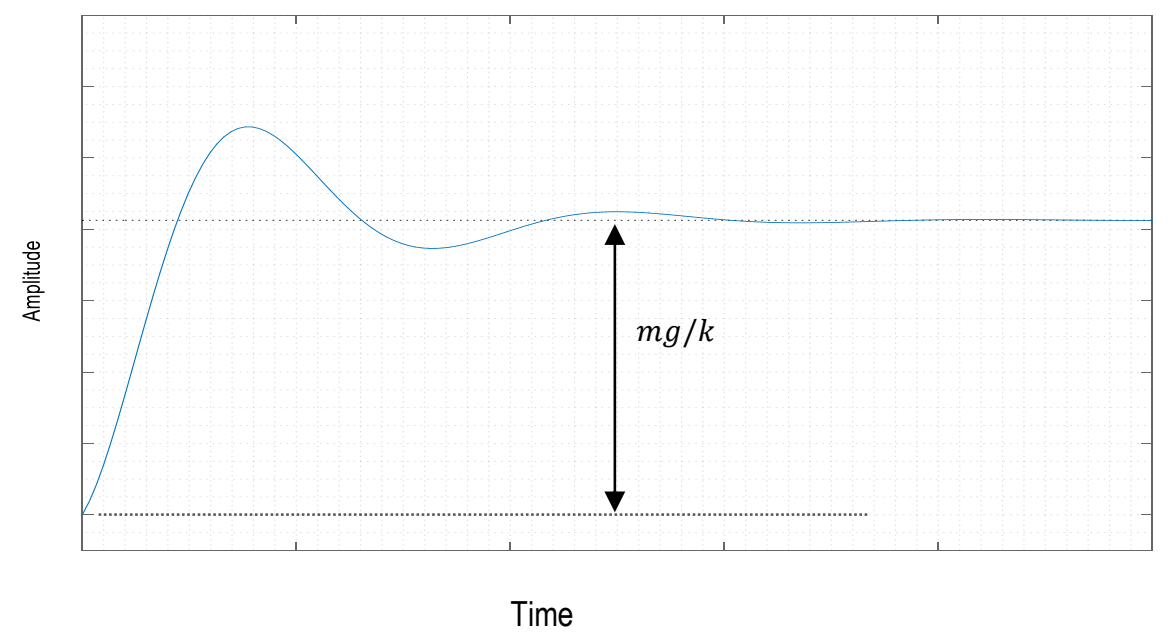

Figure (3.7) Step response to a second order differential equation

The equations and analysis done in this section ignores coupling between the two load cells in the dual cell arrangement in the existing system. It assumes the dynamic behaviour of a single load cell under the applied load.

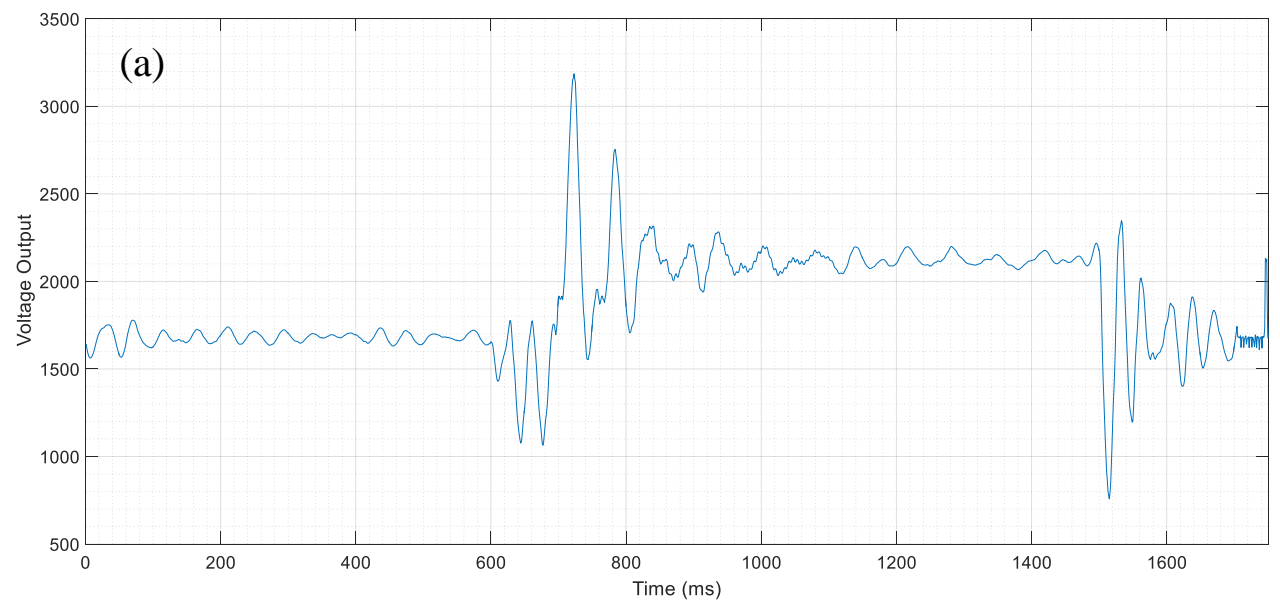




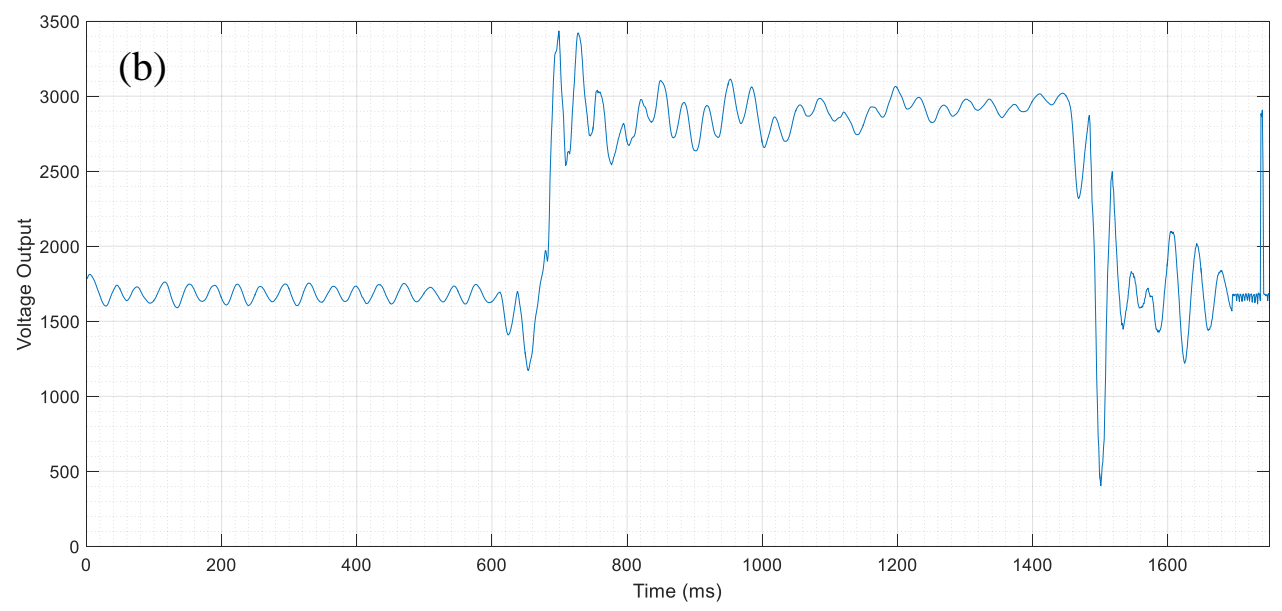

Figure (3.8): The response of one of the load cells for calibrated mass of (a) $200 \mathrm{~g}$ at $0.5 \mathrm{~m} / \mathrm{s}$ and (b) $573.1 \mathrm{~g}$ at $0.5 \mathrm{~m} / \mathrm{s}$

\subsubsection{Fifth order Butterworth filter}

The Butterworth filter designed to pass low frequency signals and attenuate the amplitude of high frequency signals. The Butterworth filter achieves flat filter gain in the pass band, at the expense of a relatively wide transition region from pass band to stop band. The sharpness of the roll-off from pass band to stop band is determined by the filter order.

Butterworth filter is an all pole filter, fifth order Butterworth filter having 5 poles spaced equidistant on a circle in the complex s-plane around the origin and having the radius of the circle equal to the cut-off frequency (Acharya et al., 2014).

The filter used in the weighing system is an analogue filter.

Properties of the Butterworth filter used in the weighing system at Compac, are not available. Therefore, the transfer function of the normalised Butterworth filter (filter gain $=1$, cut off frequency $=1 \mathrm{rad} / \mathrm{s}$ ) was used to study the effects of the Butterworth filter on the weighing signal.

The frequency response of the $5^{\text {th }}$ order of a normalised Butterworth filter, where cut-off frequency $\omega_{c}=1$ and the filter gain $=1$, is given by (Yilmas \& Tola, 2011),

$$
H_{B}(j \omega)=\frac{1}{(s+1)\left(s^{2}+0.618 s+1\right)\left(s^{2}+1.618 s+1\right)}
$$

The transfer function can be scaled up to the required cut-off frequency $\left(\omega_{c}\right)$ by replacing $\mathrm{s}$ with $s / \omega_{c}$ as below. 


$$
H_{B}(j \omega)=\frac{\omega_{c}{ }^{5}}{\left(s+\omega_{c}\right)\left(s^{2}+0.618 \omega_{c}+\omega_{c}^{2}\right)\left(s^{2}+1.618 \omega_{c}+\omega_{c}^{2}\right)}
$$

Filtered signal is produced by convolving the 'load cell output' with the impulse response of the 'filter kernel'. This also can be achieved by multiplying the transfer functions in frequency domain.

The entire weighing mechanism including the filter is represented by a transfer function shown below.

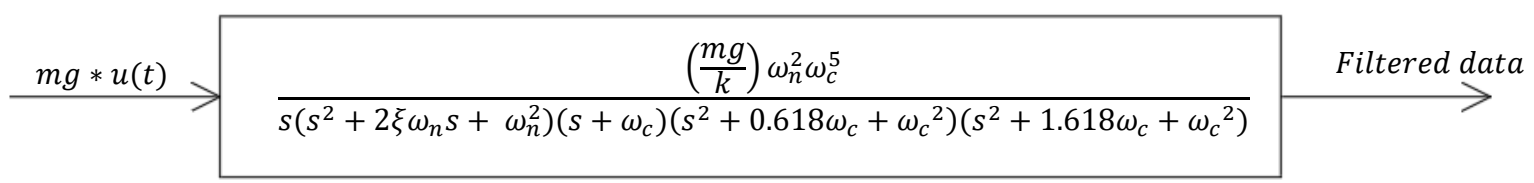

Figure (3.5): The transfer function of the weighing system including the Butterworth filter

The number of poles in the system has increased. Additional poles alter the step response of the system. The filter gain attenuates the high amplitudes of the oscillations in passband frequencies.

The graphs of filtered and unfiltered data of $200 \mathrm{~g}$ mass at $0.5 \mathrm{~m} / \mathrm{s}$ and $200 \mathrm{~g}$ at $1.5 \mathrm{~m} / \mathrm{s}$ are shown in Figure (3.8(a)) and (3.8(b)). Visualised in these graphs, is the filtered response is less oscillatory when compared with the unfiltered response as expected. It is also observed that there is a time lag between the unfiltered and filtered responses. In analogue filters, the delay is introduced by the electronic components of the filter circuit e.g. capacitors.

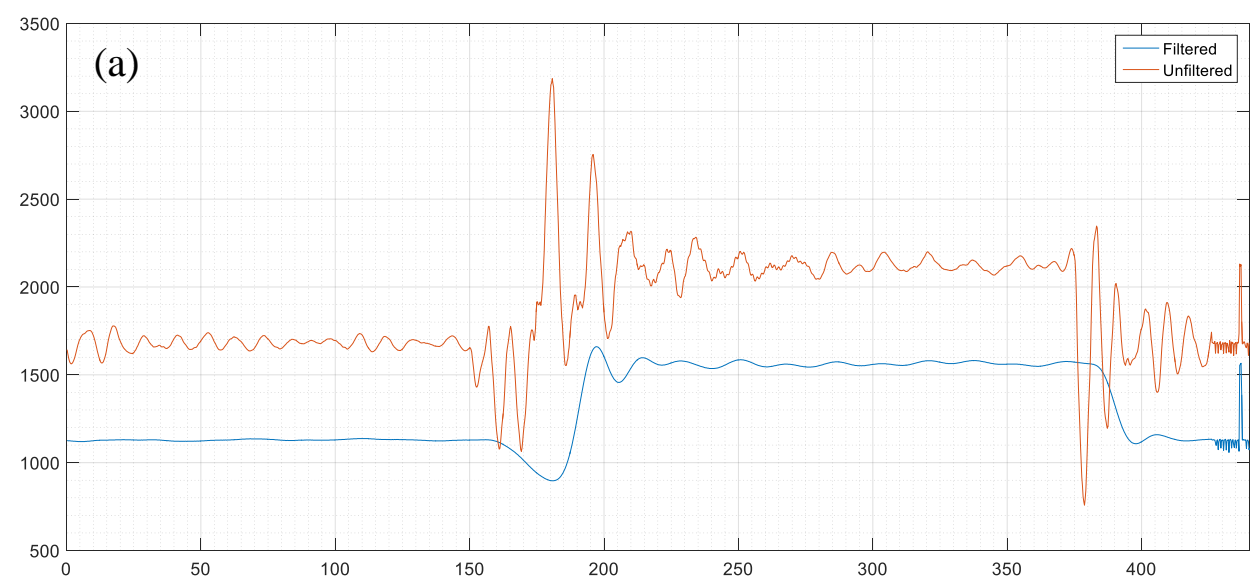




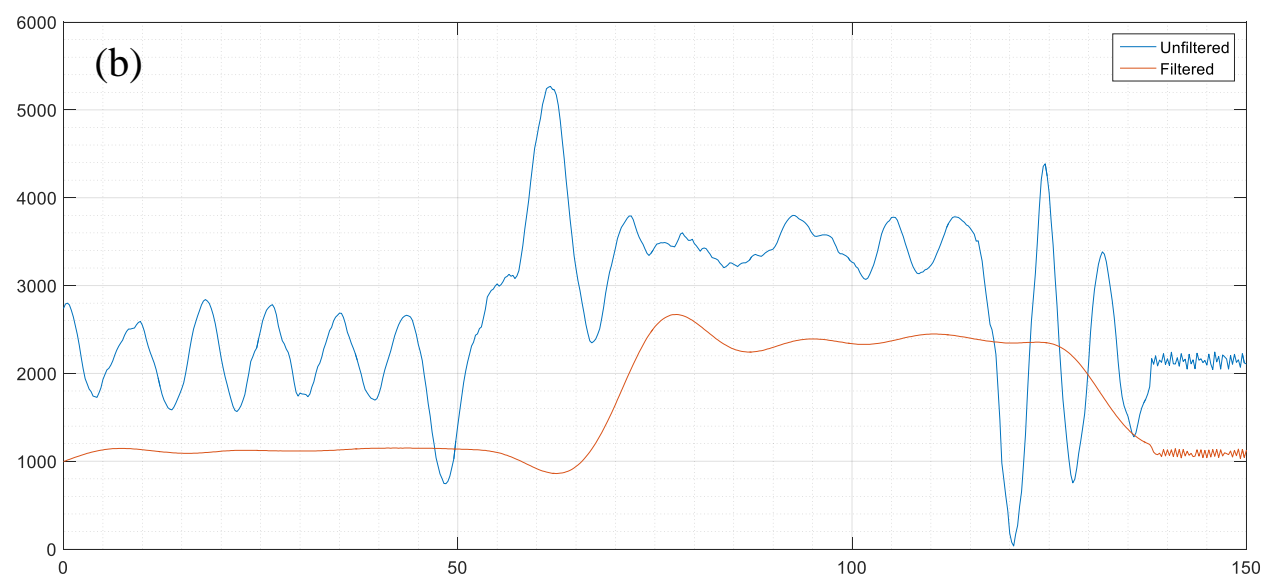

Figure (3.8): Filtered and unfiltered data (a) $200 \mathrm{~g}$ moving at $0.5 \mathrm{~m} / \mathrm{s}$ (b) $200 \mathrm{~g}$ moving at $1.5 \mathrm{~m} / \mathrm{s}$ 


\section{Chapter 4.0 Data analysis}

\subsection{Introduction}

The experimental data provided by Compac sorting Ltd are the raw output data of the analogue to digital converter (unfiltered data) and the data filtered by the fifth order Butterworth filter, analogue filter (filtered data) for each load cell of the dual load cell system described in Chapter 1, for three different fruits at various speeds. Filtered and unfiltered data are available from both load cells, for empty cups, at two different speeds.

\subsection{Preliminary observations}

Unfiltered and filtered voltage output data are examined, and the observations made are discussed in this section. The signal properties and the variation of its behaviour with the change of the input conditions are recoded below.

The speed of the conveyor belt is given in 'cups per minute'. The length of the cup is assumed to be $100 \mathrm{~mm}$ and the conveyor belt speed was converted to metres per second $(\mathrm{m} / \mathrm{s})$ values.

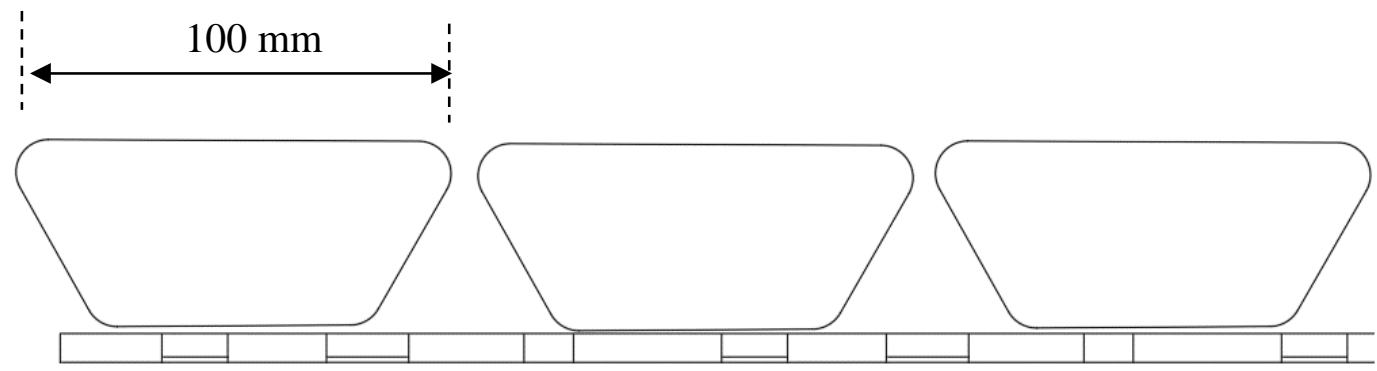

Figure (4.1): Schematic diagram of cup attached to the belt 


$$
\begin{aligned}
300 \text { cups per minute } & =30 \mathrm{~m} / \text { minute } \\
& =0.5 \mathrm{~m} / \mathrm{s}
\end{aligned}
$$

\subsubsection{Observations of data as fruits pass over the weigh table}

A number of unfiltered data sets provided by Compac sorting Equipment were examined. The plateau heights, signal times and the settling times listed in table (4.1) and (4.2) were approximate values observed from the unfiltered data versus time graphs as shown below.

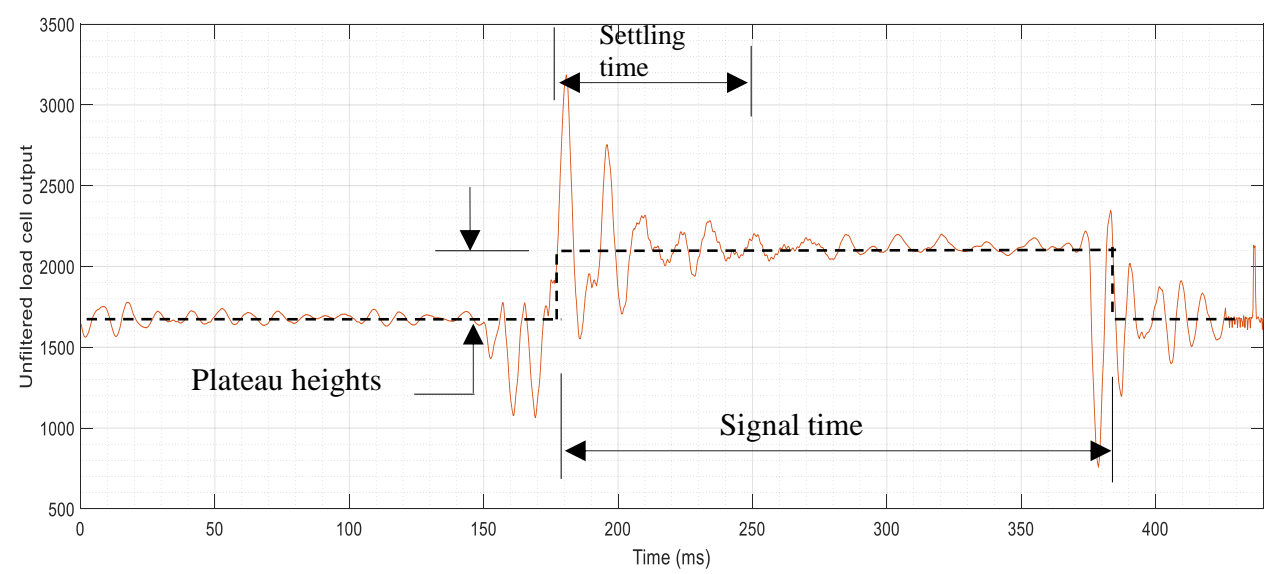

Figure (4.2): Unfiltered data as $200 \mathrm{~g}$ mass at $0.5 \mathrm{~m} / \mathrm{s}$ belt speed for one of the load cells.

The voltage signal is oscillatory before the fruit approaches the weigh table and as it passes over the weigh table. There is a clear increase in amplitude of the oscillations around 180 ms as the fruit slides on to the load cell.

When the fruit is moving at $0.5 \mathrm{~m} / \mathrm{s}$, the signal time is $200 \mathrm{~ms}$.

There is an increase in the average signal value creating a plateau around the weight to be estimated, as fruits slide on to the load cell and the signal drops as the fruit slides off the load cell. Weight of the fruit is responsible for the increase in the signal value between $200 \mathrm{~ms}$ and $380 \mathrm{~ms}$. The increase in the mean value will be referred to as the "plateau height' in this thesis and is expected to give the mass of the fruit through calibration. Some offsets are introduced to some data sets to clearly visualise the signals.

Similar graphs for the other fruits at different speed values were examined. Observations made from these graphs are summarised below. 


\begin{tabular}{|c|c|c|c|c|}
\hline Speed $(\mathrm{m} / \mathrm{s})$ & $\begin{array}{l}\text { Plateau } \\
\text { height }\end{array}$ & $\begin{array}{l}\text { Signal time on } \\
\text { LC }(\mathrm{ms})\end{array}$ & $\begin{array}{l}\text { Settling time } \\
(\mathrm{ms})\end{array}$ & $\begin{array}{l}\text { Weighing } \\
\text { time }\end{array}$ \\
\hline 0.5 & 400 & 200 & 70 & Up to $110 \mathrm{~ms}$ \\
\hline 1.0 & 450 & 100 & 50 & $40 \mathrm{~ms}$ \\
\hline 1.5 & 1100 & 60 & \multicolumn{2}{|c|}{ Does not reach a steady value. } \\
\hline
\end{tabular}

Table (4.1): Observation of signal values of $200 \mathrm{~g}$ at $0.5 \mathrm{~m} / \mathrm{s}, 1.0 \mathrm{~m} / \mathrm{s}$ and $1.5 \mathrm{~m} / \mathrm{s}$

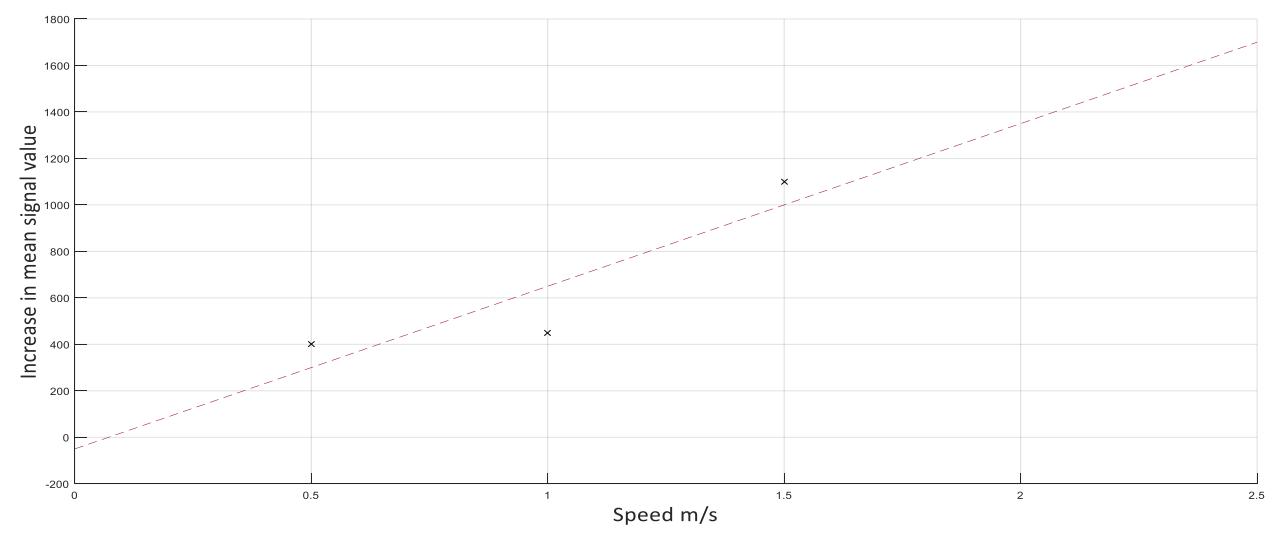

Figure (4.3) - Graph showing the variation of the plateau height of $200 \mathrm{~g}$ mass with the convevor helt sneed.

Visualised in the graph shown Figure (4.3) is an increasing trend between the 'plateau height' and the speed of the conveyor belt.

The increase of the conveyor belt speed results in shorter signal times and smaller transient response durations. At faster speeds, the averaging window used to estimate the weight of the fruit is in the former part of the transient response and the average signal values obtained will be influenced by the higher amplitudes of oscillations of the signal as the mass slides on to the weighing table.

\begin{tabular}{|l|c|c|l|l|}
\hline & $\begin{array}{l}\text { Plateau } \\
\text { height }\end{array}$ & $\begin{array}{l}\text { Signal time } \\
\text { on LC }(\mathbf{m s})\end{array}$ & $\begin{array}{l}\text { Settling time } \\
(\mathbf{m s})\end{array}$ & $\begin{array}{l}\text { Weighing time } \\
(\mathbf{m s})\end{array}$ \\
\hline $200 \mathrm{~g}$ at $0.5 \mathrm{~m} / \mathrm{s}$ & 400 & 180 & 70 & Up to $110 \mathrm{~ms}$ \\
\hline $\begin{array}{l}403.5 \mathrm{~g} \text { at } \\
1.0 \mathrm{~m} / \mathrm{s}\end{array}$ & 890 & 110 & $\begin{array}{l}\text { Raw signal is highly oscillatory right } \\
\text { through out. } \\
\text { High amplitudes reduces after 40, } 50 \\
\text { ms. }\end{array}$ \\
\hline $\begin{array}{l}573.1 \mathrm{~g} \text { at } \\
0.5 \mathrm{~m} / \mathrm{s}\end{array}$ & 1143 & 180 & $\begin{array}{l}\text { Raw signal is oscillatory right } \\
\text { through out but lower amplitudes } \\
\text { when compared to 403.5g at } 1 \mathrm{~m} / \mathrm{s} . \\
\text { High amplitudes reduces after 40 - } \\
50 \mathrm{~ms}\end{array}$ \\
\hline
\end{tabular}

Table (4.2): Observation of signal values of different masses 


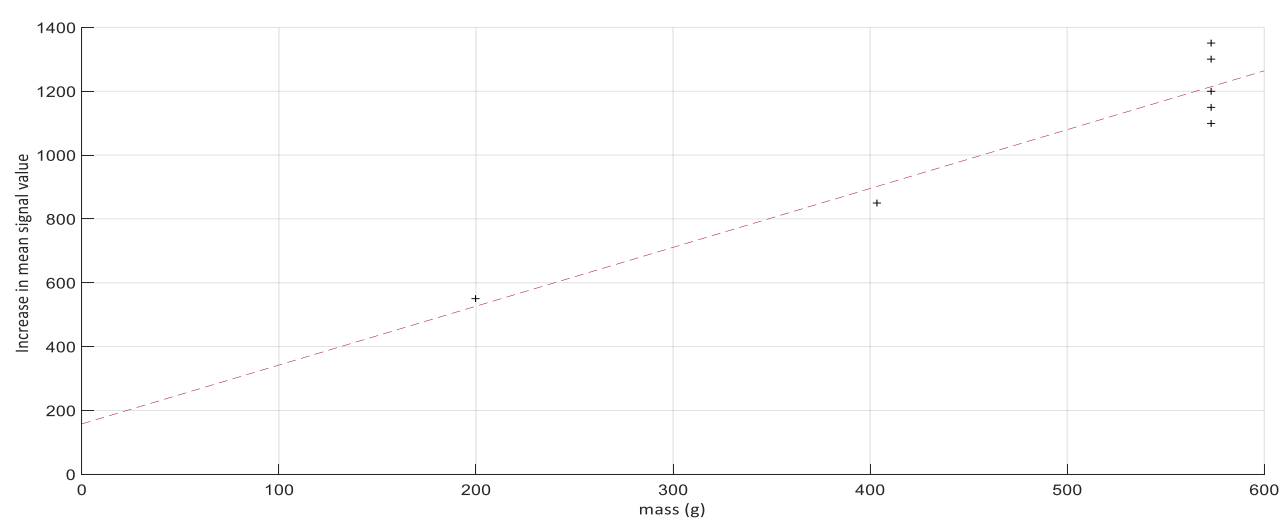

Figure (4.4): Graph showing the variation of the plateau height with the increase of mass of the fruit

The graph shown in Figure (4.4) shows an increasing trend between the average signal value and the mass of fruit.

This is an expected trend as the weight of the fruit is responsible for the plateau height.

\subsubsection{Observations of data as empty cups passing over the weighing table}

Data were available for empty cups at two belt speeds; $0.25 \mathrm{~m} / \mathrm{s}$ and $1 \mathrm{~m} / \mathrm{s}$. The graphs of signal versus the time were examined. Visualised in these graphs are;

- The difference between the mean values of the signal before it arrives at the weigh table and as it pass over the weigh table is negligible in both cases.

- Amplitudes of oscillations suddenly increase as the empty cups slide on to the weigh table. This indicates possible system vibrations as the fruit slides on to the weigh table. At the speed of $0.25 \mathrm{~m} / \mathrm{s}$, the amplitude vibrations increases to 2800 , 600 units above the mean value of 2200 units, and decays, approximately in 100 ms.

Presence of external vibrations that last for the $1^{\text {st }}$ part of the load cell output signal is evident in these graphs. The graph of the raw signal when the empty cups moving at 0.25 $\mathrm{m} / \mathrm{s}$ is show in figure (4.5). 


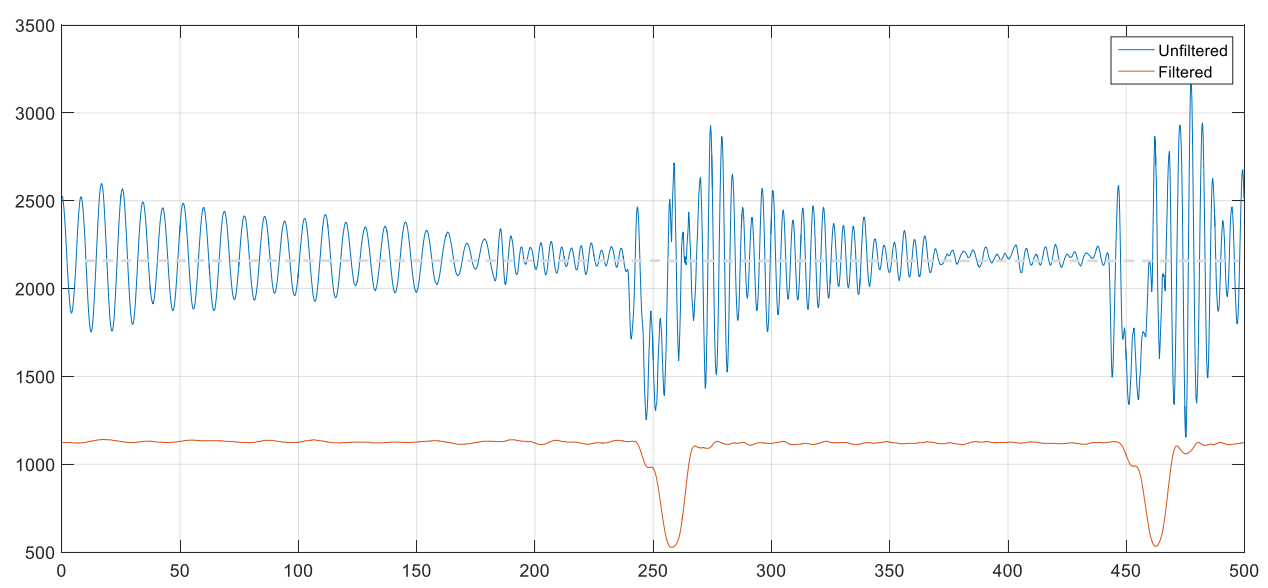

Figure (4.5): Filtered and unfiltered data as empty cups pass over the weigh table at $0.25 \mathrm{~m} / \mathrm{s}$ belt speed.

\subsection{Power spectrum analysis}

The power spectrums of the unfiltered signals were studied to understand the relationship between the system frequency for each fruit at a given speed and the mass of the fruit.

Power spectral density (PSD) function is a way of illustrating the variation of signal power with frequency (Wilson, 2016). The graph of 'power density' in the signal versus 'frequency' is a representation of the magnitude of various frequency components of a signal.

Power is proportional to the square of the signal amplitude (Wilson, 2016).

There are many methods for estimating power spectral density. Fast Fourier Transform (FFT) was the method employed in estimation of PSD (Wilson, 2016).

Similar to Fourier analysis which decomposes a function of known period into a sum of sine and cosine functions of different frequencies and amplitudes, Fourier transform is a mathematical method that decomposes a non-periodic signals and time series data of unknown period into a number of sinusoidal functions having different frequencies and amplitudes (Wilson, 2016). It measures the fluctuations in a signal or a data series by comparing it with its constituent sinusoidal functions. Fourier transform represent the frequency spectrum of the non-periodic signal. If a continuous signal is sampled, it becomes a set of discrete values and Discrete Fourier Transform (DFT) is applied to find its frequency spectrum. FFT is an algorithm that computes DFT of a discrete signal efficiently. FFT clearly identifies the dominant frequency that exists in the signal (Wilson, 2016). 
Frequency spectra of the data provided was examined to obtain dominant frequency in each spectrum. Observations made are listed below.

- $\quad$ FFT analysis reveals a peak around $110-120 \mathrm{~Hz}$ in most of the cases before the fruit moves on to the load cell.

- When the $200 \mathrm{~g}$ mass is on the load cell, there is a peak of around $50 \mathrm{~Hz}$ too but this peak is still dominated by other frequencies around $50 \mathrm{~Hz}$ too. This is consistent with the behaviour of $200 \mathrm{~g}$ mass at all the three belt speeds.

- When the $403.5 \mathrm{~g}$ is on the load cell, peaks are around $50 \mathrm{~Hz}$ and $150 \mathrm{~Hz}$.

- When the $573.1 \mathrm{~g}$ is on the load cell, the peaks are around $30 \mathrm{~Hz}$ and $125 \mathrm{~Hz}$.

- These frequency distributions are very similar for data on load cell 2 too.

Visualised in these graphs, the peak frequencies varies with the speed of the conveyor speed for the same mass. The peaks in the FFT plots are also dominated by other frequencies therefore using the natural frequency method for estimating the mass of the fruit was not practically achievable in this case. FFT plot of some data sets are shown in figure (4.6).

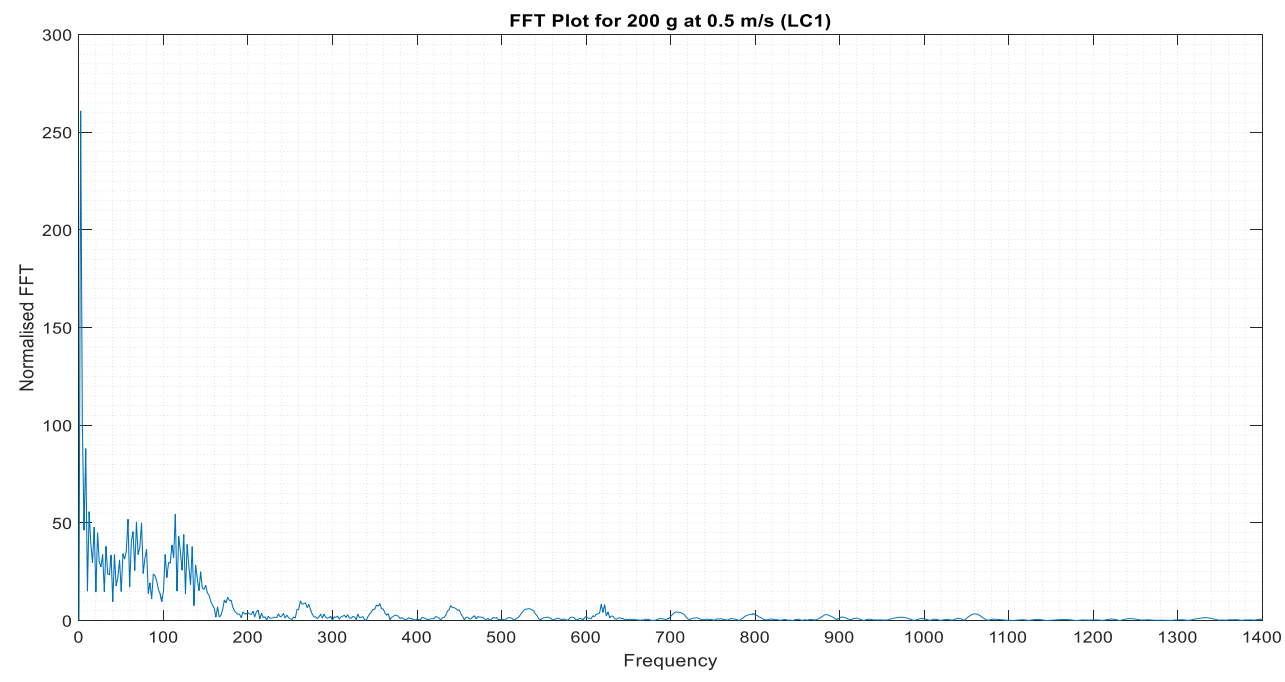




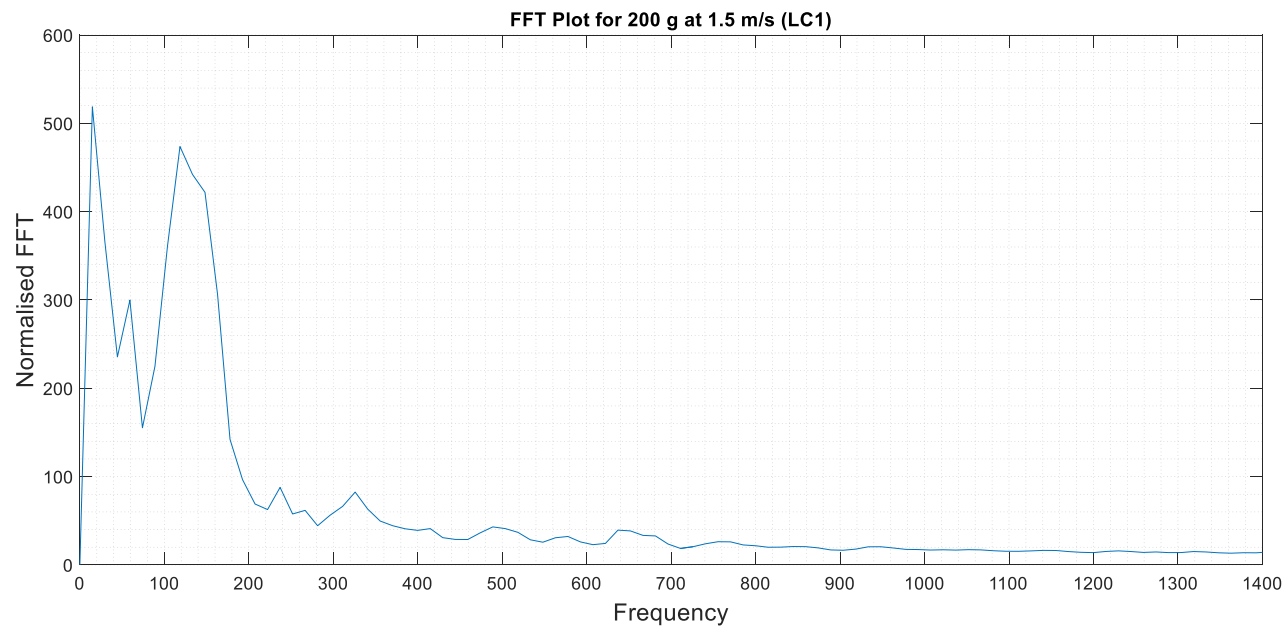

Figure (4.6): FFT plots for $200 \mathrm{~g}$ moving at $0.5 \mathrm{~m} / \mathrm{s}$ and $1.5 \mathrm{~m} / \mathrm{s}$

Frequency spectra attached in Appendix shows the FFT plots of data obtained from load cell 1 for following cases.

(i) Empty cups at $0.25 \mathrm{~m} / \mathrm{s}$ and $1.0 \mathrm{~m} / \mathrm{s}$.

(ii) $\quad 200 \mathrm{~g}$, calibrated mass at $0.5 \mathrm{~m} / \mathrm{s}, 1.0 \mathrm{~m} / \mathrm{s}$ and $1.5 \mathrm{~m} / \mathrm{s}$.

(iii) $\quad 403.5 \mathrm{~g}$ at $1.0 \mathrm{~m} / \mathrm{s}$.

(iv) $\quad 573.1 \mathrm{~g}$ at $0.5 \mathrm{~m} / \mathrm{s}$

\subsection{Data analysis using system identification method}

System identification is a methodology for building a mathematical model of dynamic systems with the use of output data as a response to a given input. The system is assumed to be a 'black box' and input and output data are used to estimate a mathematical expression that defines the system.

System identification tool (SID) in 'MATLAB' is a useful tool to study the data and predict the dynamics of the weighing mechanism. The system identification tool uses an 'Automatic non- linear line search algorithm' to estimate the transfer function of a given system. The process of identifying a data driven system function requires input and output data, a model structure.

A step function (representing the constant weight applied on the load cell) as the input and unfiltered output data of the load cell as the output of the system. The model structure used is the 'transfer function'. 
Results obtained from the simulations shows that the transfer function of the weighing mechanism varies with the mass as well as with the change of belt speed. These transfer functions obtained were higher order functions instead of the expected second order systems.

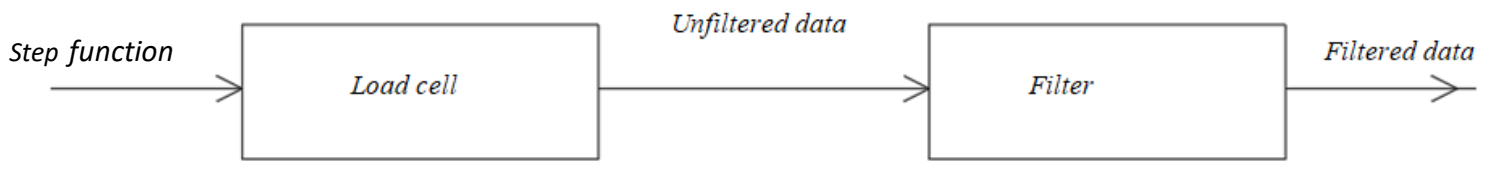

Figure (4.7): Data flow through the weighing system

\section{System identification analysis of the load cell}

A unit step function was used to represent the constant force applied on the load cell during the period that fruit is on the plate attached to the load cell. Output data was the unfiltered data provided by Compac.

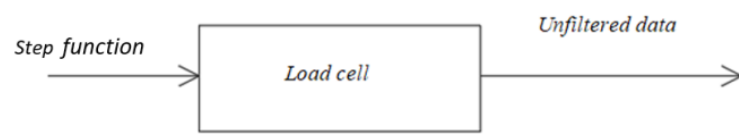

Figure (4.8): Data flow through the load cell

The tests were carried out using the three different set of output data, i.e. $200 \mathrm{~g}$ at $0.5 \mathrm{~m} / \mathrm{s}$, $200 \mathrm{~g}$ at $1.0 \mathrm{~m} / \mathrm{s}$ and $200 \mathrm{~g}$ at $1.5 \mathrm{~m} / \mathrm{s}$.

The time plots used for $200 \mathrm{~g}$ moving at $0.5 \mathrm{~m} / \mathrm{s}$ are shown in the figure (4.9).
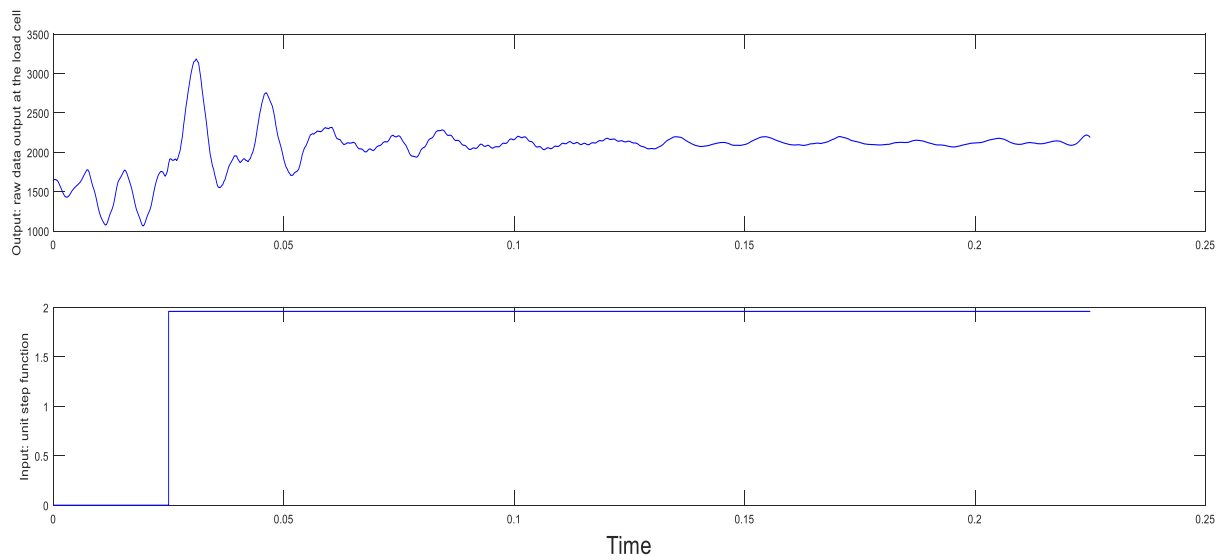

Figure (4.9): Time plot of $200 \mathrm{f}$ at $0.5 \mathrm{~m} / \mathrm{s}$ 
As shown in figure (4.10), when a second order transfer function was used as the model structure, best fit yielded was $6.04 \%$ (shown in blue). The highest percentage of best fit obtained was $59.23 \%$ (shown in red) and the transfer function has three zeros and five poles. Shown in black is the plot of unfiltered data at $200 \mathrm{~g}$ at $0.5 \mathrm{~m} / \mathrm{s}$.

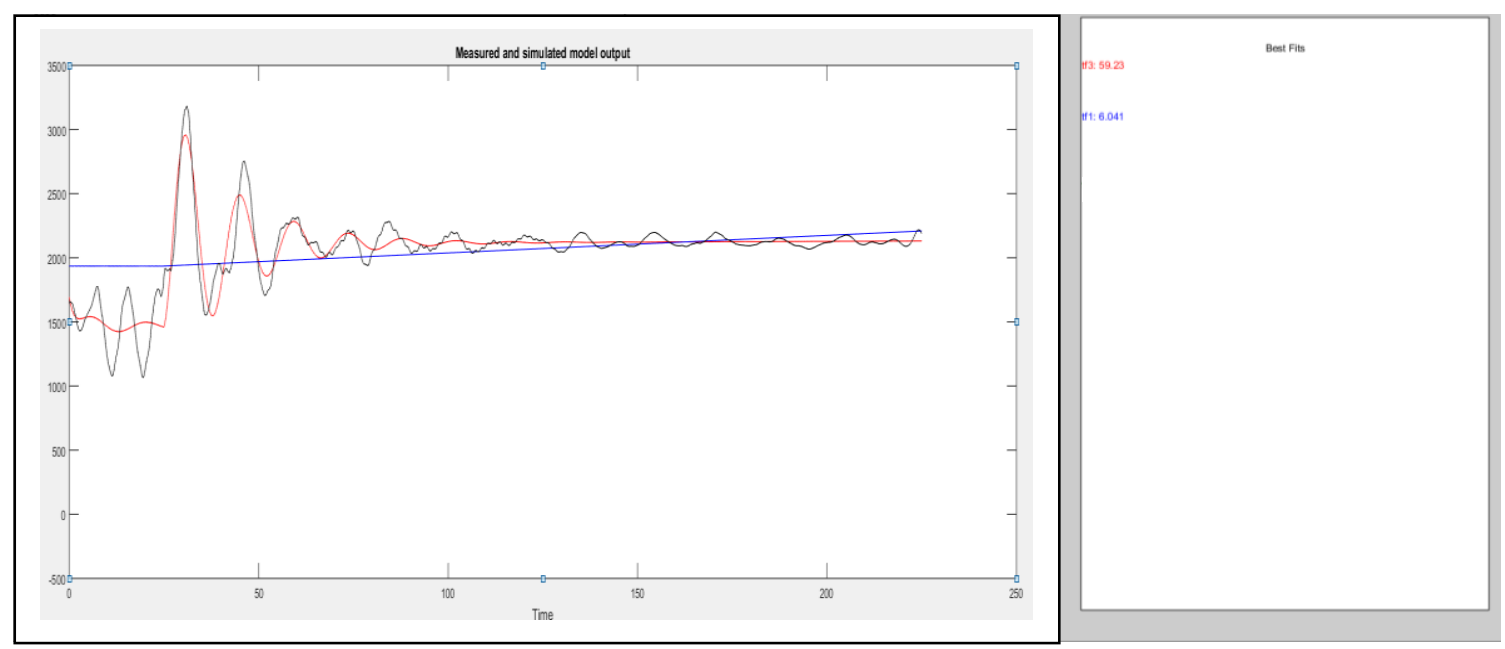

Figure (4.9): Model plots of the step response of second order transfer function (blue), $5^{\text {th }}$ order transfer function (red).

Tests carried out on other data sets showed similar results. The best fitting transfer functions obtained for data at higher speeds were of higher order. The higher order of the transfer function implies a higher order weighing systems.

The existing weighing system is a dual load cell system. The fruit is carried over the weighing systems in a fruit carrier which has 4 contact points, two on each side. The carrier moves along a steel plate which is mounted on load cells. One possible reason for a higher order transfer function is the coupled load cells that transmit oscillations and vibrations from one to the other. 


\section{Chapter 5.0}

\section{Development of the filter}

\subsection{Introduction to Kalman filter}

The Kalman filter is an optimal estimation algorithm named after the Rudolf E. Kalman who developed the algorithm (Kalman, 1960). The recursive estimation algorithm was published in 1960 and has been vital in implementation of navigation systems, radar systems, control systems, computer graphics and many more (Goldstein, 2004). The Kalman filter was originally developed for control engineering applications and was regarded as a control theory (Bernhard \& Deschamps, 2017). However, it is based on estimation theory; consequently also viewed as a signal processing theory.

The optimal state estimator algorithm essentially estimates the unmeasured states of linear dynamic systems or processes from noisy observations. Extensions of the algorithm were also developed for non-linear systems (Welch \& Bishop, 2006).

The states (parameters of the system of interest) of a system are often directly not measurable but are estimated on indirect measurements. The measurements, or a series of measurements in some cases, are then translated into a state using known relationship between the measurements and the state. The measurements taken using the sensors contain measurement noise and other uncertainties and it is a challenging task to estimate states precisely and accurately.

With use of the laws of physics, system dynamics may be included in the state estimation process. However, the system dynamics are not completely known and often associated with deviations and errors.

In the Kalman filter approach, partial uncertainty in both measurement and system dynamics are expressed in terms of probability. The information from both measurements and system dynamics are then assimilated in a statistical way to 'filter out' the noise and obtain the best estimate of the system state. It is an efficient recursive filter that minimises the mean square 
error between the estimated state and the expected state based on a state space model of the system.

The state space model consists of two equations; the state equation containing the information of system dynamics and the measurement equation providing the relationship of the observations to the state to be estimated (Doraiswami, 2014). Both contain uncertainties that have known probability distributions. The state equation projects the current state based on the physics of the system and measurement update adjusts the projected estimate by an actual, current measurement.

The state equation, the covariance of probability distributions of the process uncertainty, and the measurement uncertainty play a crucial role in the performance of the Kalman filter.

The linear dynamic systems can be modelled as continuous time systems by using differential equations or discrete time systems with the use of difference equations. The Kalman filter algorithm was originally developed for discrete time systems and later extended for continuous time systems. The Kalman filter algorithm developed for continuous time systems was presented as the 'Kalman - Bucy filter' and first published in 1962.

The Kalman filter algorithm can be used as a prediction algorithm, a filter or a 'smoother' depending on the method used for estimating the state from the measurements (The State Space Models, n.d).

It is a prediction algorithm if the current state, $x_{k}$ is estimated from previous measurements, $y_{k-1}, y_{k-2}$. It is a filter if the current state, $x_{k}$, estimated from current and previous measurements, $y_{k}, y_{k-1}$. It is a smoother if the current state, $x_{k}$, is estimated from $y_{n}$, where $\mathrm{n}>\mathrm{k}$.

There are two approaches to deriving the Kalman filter: Deterministic approach and probabilistic approach (Doraiswami, 2014). The derivation of the filter algorithm using the deterministic approach, is explained in detail in section 5.2.

\subsection{Kalman filter theory and algorithm}

The mathematical model of the system is expressed in state-space form that can express univariate as well as multivariate systems. The state space model can also handle time variant and time invariant systems. 


\subsubsection{Kalman filter algorithm for a single variable system}

The system dynamics of a dynamical system that evolves in time is represented in a state transition equation or the system equation (Welch \& Bishop, 2006):

$$
x_{t+1}=F_{t} x_{t}+B_{t} u_{t}+w_{t} ; w_{t} \sim N\left(0, \sigma_{w}^{2}\right)
$$

where $x_{t}$, a scalar, is the state or the system parameter to be estimated at time $t$.

$F_{t}$ is a state transition value that describes the effect of the current state $x_{t}$ on $x_{t+1}$, the updated state.

$u_{t}$ is a control input disturbance to the system, and $B_{t}$ is the effect of the control input disturbance, $u_{t}$ on the state $x_{t}$.

$w_{t}$ is the process noise term associated with $x_{t}$. The process noise is assumed to be Gaussian with zero mean and a known variance of $\sigma_{w}^{2}$.

The measurement equation is;

$$
y_{t}=H_{t} x_{t}+v_{t} ; v_{t} \sim N\left(0, \sigma_{v}^{2}\right)
$$

where $x_{k}$ is a current state, $H_{t}$ is a transformation coefficient that maps the system states into the measurement domain, $v_{t}$ is observation noise distributed according to Gaussian distribution with zero mean and a known variance of $\sigma_{v}^{2}$.

$v_{t}$ and $w_{t}$ are uncorrelated so that $E\left[v_{j}, w_{k}\right]=0$.

Both $v_{k}$ and $w_{k}$ are stochastic processes. Driven by these, $x_{t}$ also will have some randomness.

The Kalman filter algorithm improves the posterior estimate, recursively, by aiming to minimise the error between the new estimate and the predicted estimate.

There are several derivations of the filter available in the literature (Wells, 1996). Podesta (Podesta, 1994) shows the derivation of the Kalman filter estimation algorithm as outlined below. The system equation is simplified by setting $u_{t}$, the control input to zero which does not make significant changes to the derivation. 
Initial conditions of the state parameters $\left(x_{o}\right)$ is a random variable with a known probability function. This is assumed to have Gaussian distribution with mean of $\bar{x}_{o}$ and a known variance of $\sigma_{0}^{2}$.

$$
\begin{gathered}
x_{o} \sim N\left(\bar{x}_{o}, \sigma_{0}^{2}\right) \\
\text { where } \sigma_{0}^{2}=E\left[\left(x_{o}-\bar{x}_{o}\right)^{2}\right] .
\end{gathered}
$$

Equation (5.1) describes the state vector $x_{k}$ in terms of exact physical laws.

The unbiased estimate of the state is $\hat{x}_{t}$ at time $t$ is the priori estimate. An updated or improved estimate for the state vector $\left(\hat{x}_{t+1}\right)$ (the posterior estimate) can be obtained by combining the current estimate $\hat{x}_{t}$ and the current measurement of the state $y_{t}$ as follows.

The expected value is a measure of the long term average of random process (Thaeh, 2018). Hence $\hat{x}_{t}, \hat{x}_{t+1}$ and the measurement $y_{t}$ are assumed to have the same expected value of $\bar{x}$.

Based on the assumption that the predicted estimate has a linear relationship with its previous estimate and the previous measurement,

$$
\hat{x}_{t+1}=k_{t} y_{t}+k_{t}^{\prime} \hat{x}_{t}
$$

Then,

$$
\hat{x}_{t+1}-x_{t+1}=k_{t} y_{t}+k_{t}^{\prime} \hat{x}_{t}-F_{t} x_{t}-w_{t}
$$

Substituting for $y_{t}$

$$
\begin{gathered}
\hat{x}_{t+1}-x_{t+1}=k_{t}\left(H_{t} x_{t}+v_{t}\right)+k_{t}^{\prime} \hat{x}_{t}-F_{t} x_{t}-w_{t}, \\
=x_{t}\left(H_{t} k_{t}-F_{t}\right)+k_{t}^{\prime} \hat{x}_{t}+k_{t} v_{t}-w_{t} .
\end{gathered}
$$

Taking the expected value of both sides,

$$
\begin{gathered}
\mathrm{E}\left[\hat{x}_{t+1}-x_{t+1}\right]=E\left[x_{t}\left(H_{t} k_{t}-F_{t}\right)\right]+E\left[k_{t}^{\prime} \hat{x}_{t}\right]+E\left[k_{t} v_{t}\right]-E\left[w_{t}\right] \\
=\left(H_{t} k_{t}-F_{t}\right) E\left[x_{t}\right]+k_{t}^{\prime} E\left[\hat{x}_{t}\right]+k_{t} E\left[v_{t}\right]-E\left[w_{t}\right]
\end{gathered}
$$

$v_{t}$ and $w_{t}$ have Gaussian probability distributions with zero mean.

$$
\begin{aligned}
& E\left[v_{t}\right]=0, \\
& E\left[w_{t}\right]=0
\end{aligned}
$$

$\hat{x}_{t}$ is assumed to be the unbiased estimate of the priori state and therefore 


$$
E\left[\hat{x}_{t}\right]=E\left[x_{t}\right]
$$

In unbiased estimation of $\widehat{x}_{t+1}$,

$$
\mathrm{E}\left[\hat{x}_{t+1}-x_{t+1}\right]=0
$$

This substitution simplifies equation (5.4) to give an expression for $k_{t}^{\prime}$,

$$
k_{t}^{\prime}=F_{t}-H_{t} k_{t}
$$

Then,

$$
\hat{x}_{t+1}-x_{t+1}=\left(F_{t}-H_{t} k_{t}\right)\left(\hat{x}_{t}-x_{t}\right)+k_{t} v_{t}-w_{t}
$$

Squaring equation (5.5) and taking the expectation on both sides,

$$
E\left[\left(\hat{x}_{t+1}-x_{t+1}\right)^{2}\right]=\left(F_{t}-H_{t} k_{t}\right)^{2} E\left(\hat{x}_{t}-x_{t}\right)^{2}+k_{t}^{2} E\left[v_{t}^{2}\right]+E\left[w_{t}^{2}\right]
$$

If $P_{t}$ is the square error of the priori estimate, $\hat{x}_{t}$, then:

$$
P_{t}=E\left[\left(\hat{x}_{t}-x_{t}\right)^{2}\right]
$$

Similarly, the error variance of the posterior estimate, $P_{t+1}$ is given by:

$$
P_{t+1}=E\left[\left(\hat{x}_{t+1}-x_{t+1}\right)^{2}\right]
$$

Substituting $P_{t}$ and $P_{t+1}$ in equation (5.6),

$$
P_{t+1}=\left(F_{t}-H_{t} k_{t}\right)^{2} P_{t}+k_{t}^{2} E\left[v_{t}^{2}\right]+E\left[w_{t}^{2}\right]
$$

$E\left[v_{t}^{2}\right]$ can be written as $\mathrm{E}\left[\left(v_{t}-0\right)^{2}\right]$ which is the variance of observation noise, $\sigma_{v}^{2}$.

$$
\text { Similarly, } E\left[w_{t}^{2}\right]=\mathrm{E}\left[\left(w_{t}-0\right)^{2}\right]=\sigma_{w}^{2} \text {. }
$$

Substituting for $E\left[v_{t}^{2}\right]$ and $E\left[w_{t}^{2}\right]$ in equation (5.7)

$$
P_{t+1}=\left(F_{t}-H_{t} k_{t}\right)^{2} P_{t}+k_{t}^{2} \sigma_{v}^{2}+\sigma_{w}^{2}
$$

In order to obtain $k_{t}$ so that the posterior error variance is minimum,

$$
\begin{aligned}
\frac{d P_{t+1}}{d k_{t}} & =0, \\
& =-2\left(F_{t}-H_{t} k_{t}\right) P_{t} H_{t}+2 k_{t} \sigma_{v}^{2}=0 \\
-2\left(F_{t}-H_{t} k_{t}\right) P_{t} H_{t}+2 k_{t} \sigma_{v}^{2} & =0
\end{aligned}
$$

This yields the optimal value of $k_{t}$,

$$
K_{t}=\frac{F_{t} P_{t} H_{t}}{\left(P_{t} H_{t}^{2}+\sigma_{v}^{2}\right)}
$$

Taking the second derivative of $P_{t+1}$ to verify if this a minimum or a maximum, 


$$
\frac{d^{2} P_{t+1}}{d^{2} k_{t}}=2 P_{t} H_{t}^{2}+2 \sigma_{v}^{2}
$$

$H_{t}^{2}, \sigma_{v}^{2}$ and $P_{t}=E\left[\left(\hat{x}_{t}-x_{t}\right)^{2}\right]$ are all positive therefore,

$$
2 P_{t} H_{t}^{2}+2 \sigma_{v}^{2}>0
$$

This implies that $P_{t+1}$ (equation 5.8) is minimum when,

$$
K_{t}=\frac{F_{t} P_{t} H_{t}}{\left(P_{t} H_{t}^{2}+\sigma_{v}^{2}\right)}
$$

Substituting $K_{t}$ in equation (5.8),

$$
\begin{aligned}
P_{t+1} & =\left(F_{t}-H_{t} \frac{F_{t} P_{t} H_{t}}{\left(P_{t} H_{t}^{2}+\sigma_{v}^{2}\right)}\right)^{2} P_{t}+\left[\frac{F_{t} P_{t} H_{t}}{\left(P_{t} H_{t}^{2}+\sigma_{v}^{2}\right)}\right]^{2} \sigma_{v}^{2}+\sigma_{w}^{2} \\
P_{t+1} & =\left[\frac{\left(F_{t}\left(P_{t} H_{t}^{2}+\sigma_{v}^{2}\right)-F_{t} P_{t} H_{t}^{2}\right.}{\left(P_{t} H_{t}^{2}+\sigma_{v}^{2}\right)}\right]^{2} P_{t}+\left[\frac{F_{t} P_{t} H_{t}}{\left(P_{t} H_{t}^{2}+\sigma_{v}^{2}\right)}\right]^{2} \sigma_{v}^{2}+\sigma_{w}^{2} \\
P_{t+1} & =\left[\frac{F_{t} \sigma_{v}^{2}}{\left(P_{t} H_{t}^{2}+\sigma_{v}^{2}\right)}\right]^{2} P_{t}+\left[\frac{F_{t} P_{t} H_{t}}{\left(P_{t} H_{t}^{2}+\sigma_{v}^{2}\right)}\right]^{2} \sigma_{v}^{2}+\sigma_{w}^{2} \\
P_{t+1} & =\frac{F_{t}^{2} \sigma_{v}^{2} P_{t}\left(P_{t} H_{t}^{2}+\sigma_{v}^{2}\right)}{\left(P_{t} H_{t}^{2}+\sigma_{v}^{2}\right)^{2}}+\sigma_{w}^{2} \\
P_{t+1} & =\frac{F_{t}^{2} \sigma_{v}^{2} P_{t}}{\left(P_{t} H_{t}^{2}+\sigma_{v}^{2}\right)}+\sigma_{w}^{2}
\end{aligned}
$$

An equivalent form of equation (5.11) is,

$$
P_{t+1}=F_{t}\left(F_{t}-k_{t} H_{t}\right) P_{t}+\sigma_{w}^{2}
$$

$\boldsymbol{K}_{\boldsymbol{t}}$ is known as 'Kalman gain' at time t, that is determined by minimising the variance of the posterior state estimation error, the gain term of the filter that stabilises the filter.

When the measurement error variance, $\sigma_{v}^{2}$ approaches zero,

$$
\lim _{\sigma_{v}^{2} \rightarrow 0} K_{t}=\frac{F_{t}}{H_{t}}
$$

When the measurement error variance, $P_{t}$ approaches zero,

$$
\lim _{P_{k} \rightarrow 0} K_{t}=0
$$

Substituting for $K_{t}, y_{t}$ and $k_{t}^{\prime}$ in equation (5.3) yields an expression for the predicted value of state, $\hat{x}_{t+1}$,

$$
\hat{x}_{t+1}=F_{t} \hat{x}_{t}+K_{t}\left(y_{t}-H_{t} \hat{x}_{t}\right)
$$


The posterior estimate error variance $P_{t+1}$ reflects the variance of the state, i.e. the probability distribution of $\hat{x}_{t+1}$ is given by

$$
\hat{x}_{t+1} \sim N\left(\hat{x}_{t+1}, P_{t+1}\right)
$$

\subsubsection{Kalman filter algorithm for multivariate systems}

The Kalman filter is often used for multivariate systems, i.e. systems with multiple states to be estimated.

Similar to the 1 - dimensional case, the algorithm used in Kalman filter will be implemented recursively, (e.g. Kalman gain, the posterior states, and posterior error covariance will be updated) using vector calculus (Podesta, 1994).

For an $n$ - dimensional system (a system with $\mathrm{n}$ states), the Kalman filter is designed to operate on systems in linear state space form, i.e.

$$
\begin{aligned}
& x_{t+1}=F_{t} x_{t}+B_{t} u_{t}+w_{t} ; \text { where } w_{t} \sim N(0, Q) \\
& y_{t}=H_{t} x_{t}+v_{t} ; \text { where } w_{t} \sim N(0, R)
\end{aligned}
$$

where the variables are defined as below.

$x_{t}:$ State vector $(\mathrm{n} \times 1)$

$F_{t}:$ State transition matrix $(\mathrm{n} \times \mathrm{n})$

$B_{t}$ : Control input matrix ( $\left.\mathrm{n} \mathrm{x} \mathrm{u}\right)$

$u_{t}:$ Input disturbance vector $(\mathrm{n} \times 1)$

$w_{t}$ : A process noise vector $(\mathrm{n} \times 1)$

$y_{t}:$ Measurement vector $(\mathrm{m} \times 1)$

$H_{t}$ : Observation matrix $(\mathrm{m} \mathrm{x} \mathrm{n})$

$v_{t}$ : Measurement noise vector $(\mathrm{m} \times 1)$

The Kalman filter algorithm for a multivariate system is summarised below.

- Obtain initial conditions for state vector $\hat{x}_{t}$ and initial error covariance $P_{t}$ respectively given by $\hat{x}_{o}$ and $P_{o}$,

- The Kalman gain is calculated from equation $K_{t}=F_{t} P_{t} H_{t}^{T}\left(H_{t} P_{t} H_{t}^{T}+R_{t}\right)^{-1}$,

- Posterior state vector is calculated from, $\hat{x}_{t+1}=F_{t} \hat{x}_{t}+K_{t}\left(y_{t}-H_{t} \hat{x}_{t}\right)$,

- Posterior estimate error covariance is calculated from the equation, 


$$
P_{t+1}=\left(F_{t}-k_{t} H_{t}\right) P_{t} F_{t}^{T}+Q_{t},
$$

- Increment $t$ and repeat the process beginning with calculation of $K_{t}$ by substituting the updated error covariance $P_{t+1}$.

\subsection{Modelling the weighing system}

The process involved in the Kalman filter algorithm can also be understood as fitting the data observed (measurements) into a curve specified by the state transition model. The weighing mechanism is modelled as a second order differential equation in previous studies. An attempt was made to use the second order differential equation as the state space model in Kalman filter algorithm as shown below.

\subsubsection{Kalman filter (Using the Second order differential equation as the system model)}

The second order differential equation has been used to represent the system dynamics. The state equation was derived from the second order differential equation.

$$
(M+m) \ddot{x}+c \dot{x}+k x=g m(u(t))
$$

An equivalent discrete time equation was developed so that the model can be used with the time series data available.

Writing this as a system of fist order difference equation using the substitution,

$$
\begin{aligned}
& x_{1}=x \\
& x_{2}=\dot{x}
\end{aligned}
$$

Then,

$$
\begin{gathered}
\dot{x_{1}}=\dot{x} \\
\dot{x_{2}}=\ddot{x} \\
\dot{x_{1}}=x_{2} \\
(M+m) \dot{x_{2}}+c x_{2}+k x_{1}=g m(u(t)) \\
\dot{x_{2}}+\frac{c}{(M+m)} x_{2}+\frac{k}{(M+m)} x_{1}=\frac{g m(u(t))}{(M+m)}
\end{gathered}
$$$$
\left[\begin{array}{l}
\dot{x_{1}} \\
\dot{x_{2}}
\end{array}\right]=\left[\begin{array}{cc}
0 & 1 \\
k /(M+m) & c /(M+m)
\end{array}\right]\left[\begin{array}{l}
x_{1} \\
x_{2}
\end{array}\right]+[g(u(t)) /(M+m)] m
$$ 
Using, $\dot{x}=\frac{\Delta x}{\Delta t}=\frac{x(t+1)-x(t)}{\Delta t}$ the equation 5.16 is re-written as,

$$
\begin{aligned}
& {\left[\begin{array}{l}
x_{1} \\
x_{2}
\end{array}\right]_{t+1}-\left[\begin{array}{l}
x_{1} \\
x_{2}
\end{array}\right]_{t}=\left[\begin{array}{cc}
0 & 1 \\
k /(M+m) & c /(M+m)
\end{array}\right]\left[\begin{array}{l}
x_{1} \\
x_{2}
\end{array}\right](\Delta t)+[g(u(t)) /(M+m)] m(\Delta t)} \\
& {\left[\begin{array}{l}
x_{1} \\
x_{2}
\end{array}\right]_{t+1}=\left[\begin{array}{l}
x_{1} \\
x_{2}
\end{array}\right]_{t}+\left[(\Delta t) k /_{(M+m)}^{0}(\Delta t) c /_{(M+m)}^{\Delta t}\right]\left[\begin{array}{l}
x_{1} \\
x_{2}
\end{array}\right]_{t}+\left[\begin{array}{c}
0 \\
m g(u(t)) /(M+m)
\end{array}\right]}
\end{aligned}
$$

The multivariate state transition equation can be written in the form of,

$$
\left[\begin{array}{l}
x_{1} \\
x_{2}
\end{array}\right]_{t+1}=\left[\begin{array}{cc}
1 & \Delta t \\
(\Delta t) k /(M+m) & (1+(\Delta t) c /(M+m)
\end{array}\right]\left[\begin{array}{l}
x_{1} \\
x_{2}
\end{array}\right]_{t}+\left[\begin{array}{c}
0 \\
m g(u(t)) /(M+m)
\end{array}\right]+\left[\begin{array}{l}
w_{1} \\
w_{2}
\end{array}\right]
$$

where $w_{1}$ and $w_{2}$ are noise associated with $x_{1}$ and $x_{2}$ respectively.

The only measurement observed is the output voltage of the loadcell which is proportional to the displacement of the loadcell $\left(x_{1}\right)$. Therefore the measurement equation will be in the form of,

$$
\left[\begin{array}{l}
y_{1} \\
y_{2}
\end{array}\right]=\left[\begin{array}{ll}
1 & 0 \\
0 & 1
\end{array}\right]\left[\begin{array}{l}
x_{1} \\
x_{2}
\end{array}\right]+\left[\begin{array}{ll}
1 & 0 \\
0 & 1
\end{array}\right]\left[\begin{array}{l}
v_{1} \\
v_{2}
\end{array}\right]
$$

where $v_{1}$ and $v_{2}$ are noise associated with $y_{1}$ and $y_{2}$ respectively.

In summary, the state space model that represents the system dynamics will be as follows.

$$
\begin{aligned}
& {\left[\begin{array}{l}
x_{1} \\
x_{2}
\end{array}\right]_{t+1}=\left[\begin{array}{cc}
1 & \Delta t \\
(\Delta t) k /(M+m) & (1+(\Delta t) c /(M+m)
\end{array}\right]\left[\begin{array}{l}
x_{1} \\
x_{2}
\end{array}\right]_{t}+\left[\begin{array}{c}
0 \\
(u(t)) /(M+m)
\end{array}\right] m(\Delta t)+\left[\begin{array}{l}
w_{1} \\
w_{2}
\end{array}\right]} \\
& {\left[\begin{array}{l}
y_{1} \\
y_{2}
\end{array}\right]=\left[\begin{array}{ll}
1 & 0 \\
0 & 1
\end{array}\right]\left[\begin{array}{l}
x_{1} \\
x_{2}
\end{array}\right]+\left[\begin{array}{ll}
1 & 0 \\
0 & 1
\end{array}\right]\left[\begin{array}{l}
v_{1} \\
v_{2}
\end{array}\right]}
\end{aligned}
$$

By comparing the derived state transition equation with equation (5.14), the state transition matrix,

$$
F_{t}=\left[\begin{array}{cc}
1 & \Delta t \\
(\Delta t) k /(M+m) & (1+(\Delta t) c /(M+m)
\end{array}\right]
$$


As explained in section 5.2.2, the Kalman filter algorithm begins with calculating the Kalman gain $k_{t}$ which requires the knowledge of the system such as initial conditions, state transition matrix $F_{t}$ and observation matrix $H_{t}$.

The state transition equation contains the description of nominal system dynamics including $k$ and $c$ which can be determined empirically. The difficulty in this approach, however, is having the unknown variable ' $\mathrm{m}$ ' (the mass to be determined) in the state transition equation. The Kalman filter algorithm cannot be implemented to estimate the state $x_{1}$ with known parameters in the state transition equation.

\subsubsection{Kalman filter ( 1 - D, Discrete-time model $)$}

In a different approach, a data driven, time series model was developed and 1-dimensional Kalman filter algorithm was used to estimate the optimum steady state of the time series data.

The process followed is explained in this section.

Data provided by Compac are voltage output of the load-cells sampled at regular intervals of $0.25 \mathrm{~ms}$. A plot of voltage data versus time shows the voltage output of an unloaded loadcell followed by a plateau around the actual weight.

The proposed method uses a selected set of data; data showing a response to the weight of the fruit.

Two optical position sensors are to be used to start and end weight sampling. When the first sensor is blocked by the cup, the signal for collection of weight data is triggered and when the second sensor is blocked, the weight sampling is to be ended. The data sampled between the sensor signals is used in weight estimation.

A Discrete time state space model was employed for time series data sampled at regular intervals, and the Kalman algorithm was used as a predictor.

\subsubsection{Developing a state- space model for selected data.}

The general linear Gaussian state space model can be written in a variety of ways (Durbin $\&$ Koopman, 2012). The form of the model used in developing the state space model in this application is presented in equation (5.1) and (5.2).

Observed in the graphs shown in figures 5.1 and 5.2, the individual plot displays some oscillatory pattern corrupted with noise. However, the oscillations do show common 
properties (frequency, periodic time) for all the plots. Noisy, random oscillations regress towards a steady state.

The distribution could be described as a combination of deterministic and stochastic processes. However, the process is considered as stochastic in order to develop a common state space model for all the data sets provided, i.e. voltage data of different fruits moving at various speeds.

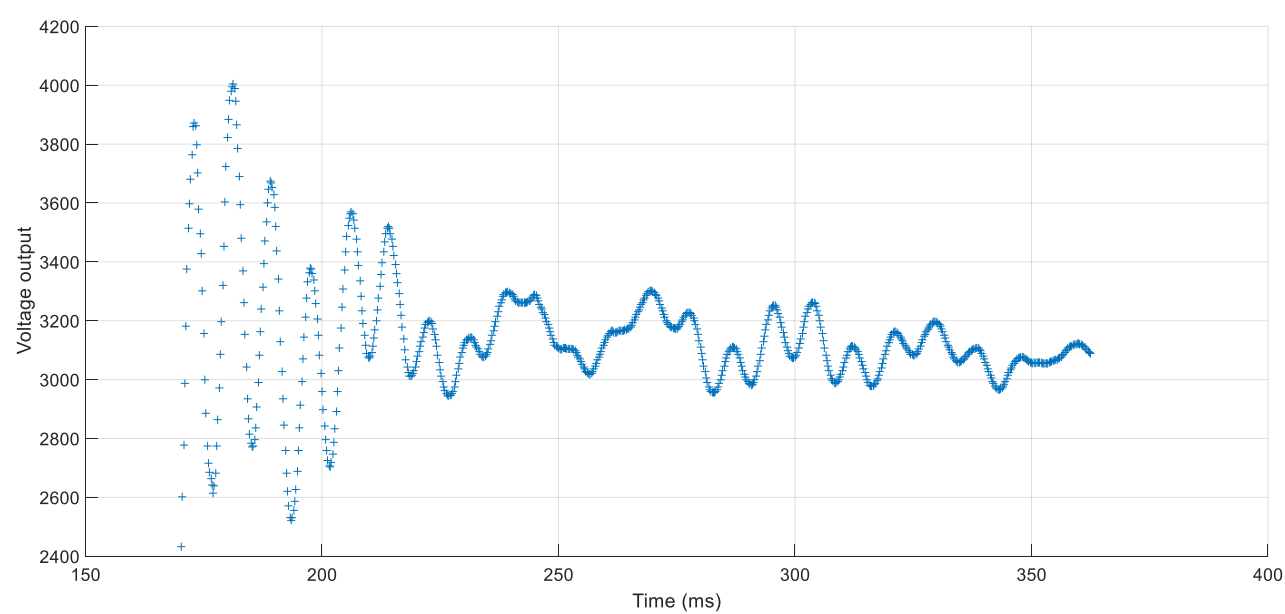

Figure 5.1: Scatter plot showing selected data of $573.1 \mathrm{~g}$ weight moving at $0.5 \mathrm{~m} / \mathrm{s}$

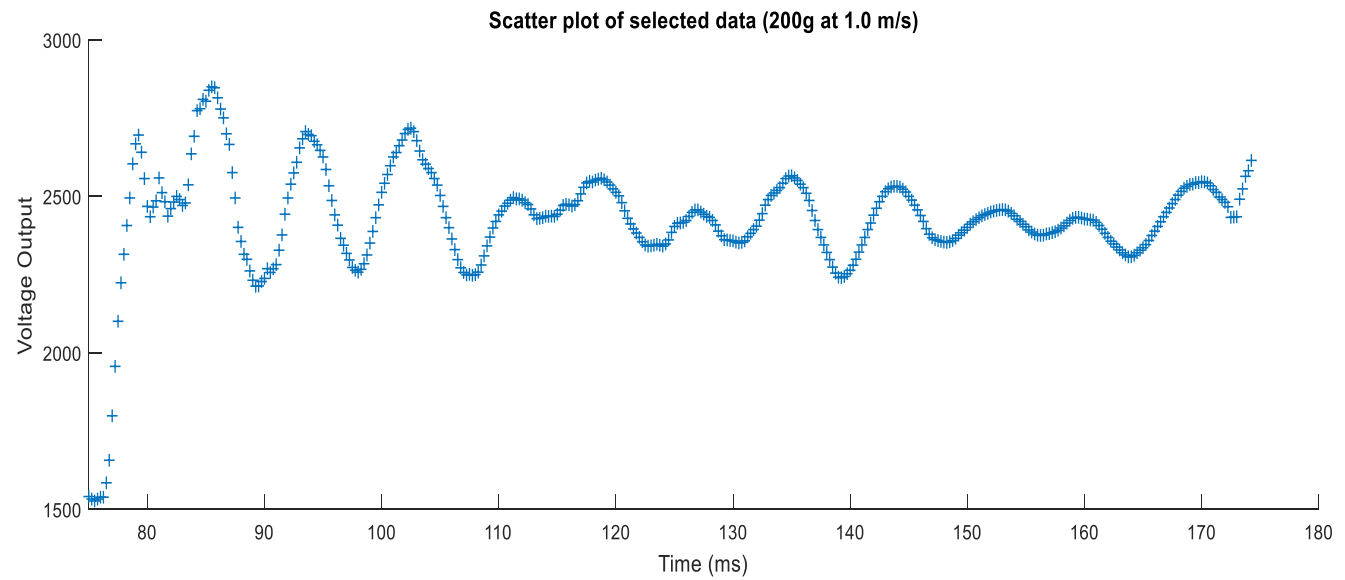

Figure 5.2: Scatter plot showing selected data of $573.1 \mathrm{~g}$ weight moving at $0.5 \mathrm{~m} / \mathrm{s}$ $\mathrm{Eq}$

Figure (5.2) shows the graph of unfiltered data for $200 \mathrm{~g}$ moving at $0.5 \mathrm{~m} / \mathrm{s}$. Visualised in this graph, the oscillatory data regresses towards constant state corrupted with some oscillations. Explained in section 3.2.1, the signal reaches a constant state of $\mathrm{mg} / \mathrm{k}$ as the time progresses towards the end of the signal time. 
Marked with black, dashed line is a hand-drawn approximation to illustrate the underlying constant state. It is also the simplified response that shows the change in voltage when the load cell is loaded with a fruit (mass).

The data was separated into two sets:

Between $\mathrm{A}$ and $\mathrm{B}$, unloaded response

Between C and D, loaded response (constant state of the step response).

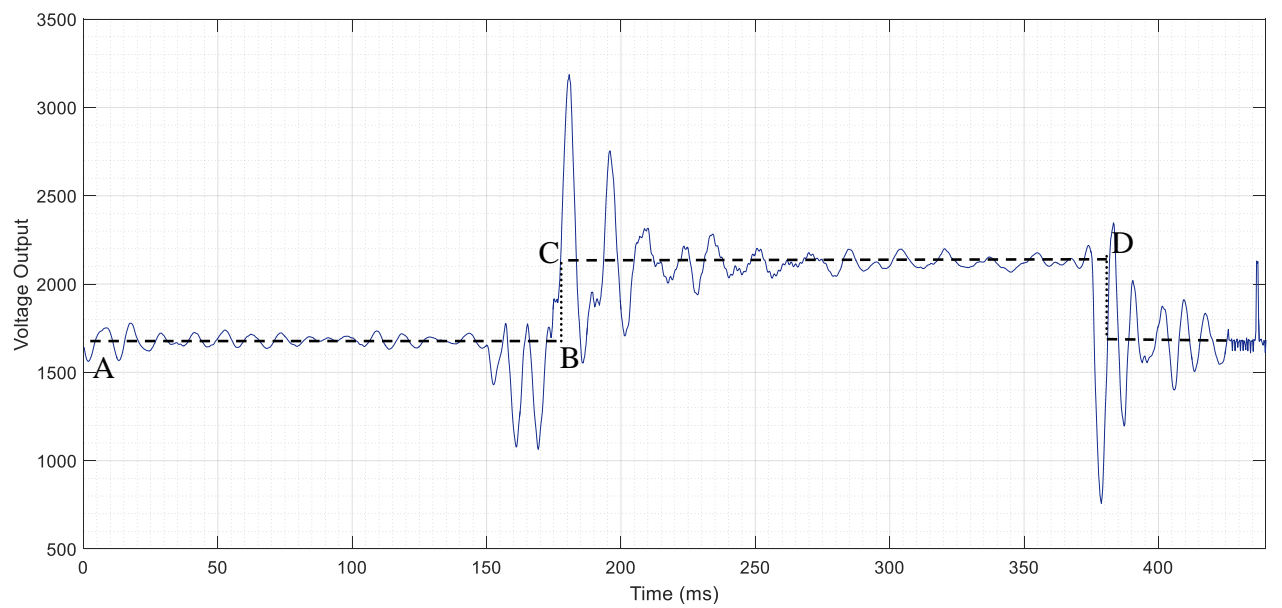

Figure 5.2: Graph of unfiltered data for $200 \mathrm{~g}$ moving at $0.5 \mathrm{~m} / \mathrm{s}$

The loaded and unloaded responses were treated as two sets of time series data. Steady state value of each was estimated separately, and the difference between the two steady state values can be used to estimate the mass of the fruit.

The model proposed does not capture the system dynamics at the level of the second order differential equation (2.1). The attempt is to estimate the underlying constant states in each case of 'unloaded response' and 'loaded response'.

In 'loaded' conditions, the underlying constant state is proportional to the mass to be estimated (equation (3.3)).

The state is assumed to be constant in the absence of any external inputs.

Explained below is the development of state space model that can be used with the 1-dimensional Kalman filter algorithm.

It is also assumed that there are no effects of external disturbances while the fruit is on the weighing table, therefore,

$$
u_{t}=0
$$


The model represent the constant state where the time update is $x_{t+1}=x_{t} \quad\left(\right.$ i. e. $\left.F_{t}=1\right)$ and the process noise variance $\sigma_{w}$ is set to zero.

Hence the state transition equation is a constant dynamic model,

$$
x_{t+1}=x_{t}+w_{t} ; w_{t}\left(0, \sigma_{w}\right), \text { where } \sigma_{w}=0,
$$

By setting the process noise variance to zero, perfect constant dynamics is assumed, i.e. It is assumed that there are no mismatches between the model and the expected state.

The state transition equation simplifies to a constant dynamic model of,

$$
x_{t+1}=x_{t}
$$

The system model represents a linear ${ }^{6}$, time invariant ${ }^{7}$ system.

\subsubsection{Measurement equation}

The measurement equation of the 1- dimensional state space model was given by equation (5.2) in section 5.2.

$y_{t}$ is the scaler output, the measured output voltage at the loadcell and $x_{t}$ is the voltage as the state to be estimated. It is assumed that $F_{t}=1$. The equation (5.2) simplifies to;

$$
y_{t}=x_{t}+v_{t}: v_{t} \sim N\left(0, \sigma_{v}\right)
$$

The difference between $y_{t}$ and $x_{t}$ is the noise associated with the measurements, i.e. any uncertainties of the measurement. The measurement uncertainty defines an interval around the measured value and the true value of the measurand lies within the interval with some probability. (Leito, n.d.)

In summary, the state space model proposed is,

$$
\begin{aligned}
& x_{t+1}=x_{t} \\
& y_{t}=x_{t}+v_{t}: v_{t} \sim N\left(0, \sigma_{v}\right)
\end{aligned}
$$

The systems with zero process noise are also known as 'output error' models.

\footnotetext{
${ }^{6}$ The output is has a linear relationship to the input.

${ }^{7}$ The output for particular input does not depend on the time of application of the input.
} 


\section{Kalman filter convergence}

Observability measures the ability of the measurements (sensor configurations) to provide all the information necessary to estimate all the states of the system and implies that the error covariance remains bounded.

Controllability of the system model measures if the state variable of the state space model can be controlled from the input to achieve the desired output. The two conditions can be tested using two tests offered in control theory. (Heidelberg, 2011. pp 275 - 297).

If the state space model of an $n$-dimensional system is given by,

$$
\begin{gathered}
x(t+1)=F * x(t)+B * u(t) \\
y(t)=C * x(t)+v(t)
\end{gathered}
$$

The $n \times n$ matrix $\left(B, F B, F^{2} B, \ldots . F^{n} B\right)$ is called the controllability matrix. The condition for controllability is that the system is completely controllable if and only if that rank of the controllability matrix is $n$.

In a similar manner, the system is observable if and only if the rank of the observability matrix,

(C, $C F, C F^{2}, \ldots . C F^{n-1}$ ) is equal to $n$ (Heidelberg, 2011. pp $275-297$ ).

The proposed model presented by equations (5.15) and (5.16) is an output error model where the process noise is zero. Zhang (2017) discusses the convergence properties of output error models and states that the optimal Kalman filter for 'output error' systems is stable (converge) if the complete observability conditions are met. This is in addition to the requirement that all the computed variables are bounded for any recursive algorithm running in real time.

For the state space model proposed in equation (5.15) and (5.16),

The number of states to be estimated $=1$

The rank of the observability matrix $(C)=1$.

The model satisfies the observability conditions.

As shown below, this is also computed in MATLAB which returns the same results. 


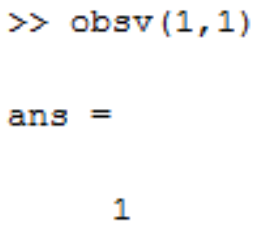

The boundedness of the computed variables, i.e. error variance, Kalman gain will be tested for each case for optimality of the Kalman filter output.

\subsubsection{Measurement noise modelling}

The measurement noise model represents the probability distribution of the measurement uncertainty which depends on the sensor. Therefore a stationary measurement noise model with constant variance is assumed. It is determined by repeating a measurement and computing the mean and standard deviation of the results ("What is the uncertainty of my sensor" n.d.).

The load cells provide accurate readings with an estimated percentage error in the range of $\pm 0.15 \%$. However, there are some factors affecting the accuracy of load cell readings. Nonlinearity of the calibration curves, hysteresis, environmental forces (vibrations) and sensitivity to temperature changes are some common reasons for measurement uncertainty (“Five factors that can affect your weighing system's accuracy", n.d.).

Exact specifications of the load cells used in the system are unavailable. The measurement noise model is developed as explained below.

Data obtained for $200 \mathrm{~g}$ moving at $0.5 \mathrm{~m} / \mathrm{s}$, which yields satisfactory weight estimates, was used as the base for estimating the uncertainty in measurements.

\section{For loaded region (between C and D in Figure (5.1)),}

Instrumentation error of the load cell is taken to be $\pm 0.2 \%$

For $200 \mathrm{~g}$ at $0.5 \mathrm{~m} / \mathrm{s}$,

At loadcell1, voltage output when loaded $\quad=2150$

$\pm 0.2 \%$ of $2150 \quad= \pm 4.3$

Standard deviation of the measurement distribution $=4.3$

Variance of the measurement distribution $\quad=18.5$ 
The measurement noise is assumed to have a Gaussian distribution. The mean value of the error distribution is zero with a variance of 18.5 .

\subsection{Implementation of the Kalman filter algorithm}

Implementation of the Kalman filter algorithm to obtain estimation for the steady state is explained below using time series data for $200 \mathrm{~g}$ at $0.5 \mathrm{~m} / \mathrm{s}$ for both loadcells.

The flowchart in figure (5.8) shows the implementation of the Kalman algorithm on the state space model suggested (Equation (5.15) and (5.16) in section 5.3.3.

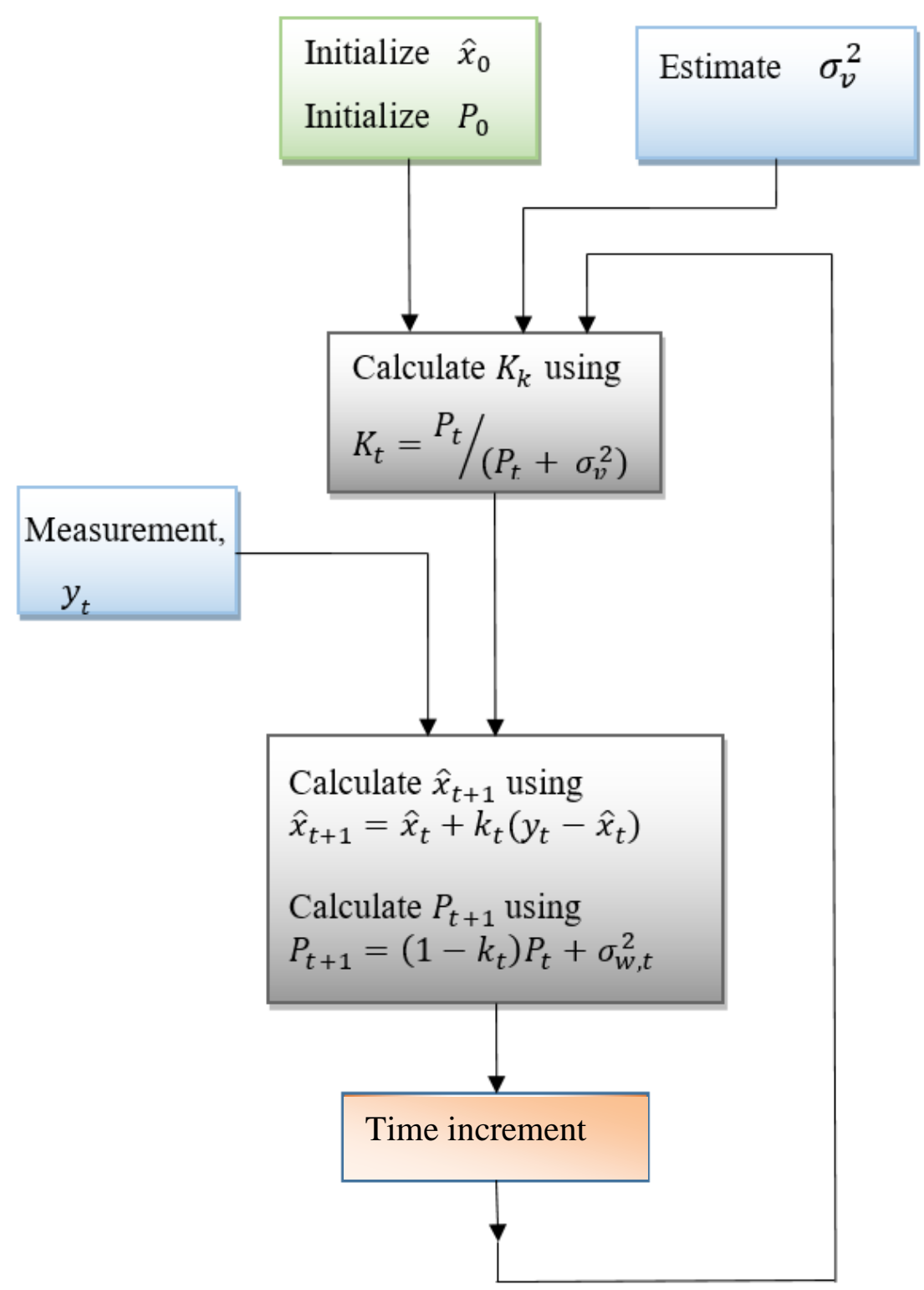

Figure 5.2: Implementation of Kalman algorithm 


\section{Initial estimations}

Figure 5.3 shows the plot of the selected data of $200 \mathrm{~g}$ at $0.5 \mathrm{~m} / \mathrm{s}$ for loadcell1 between $175 \mathrm{~ms}-375 \mathrm{~ms}$.

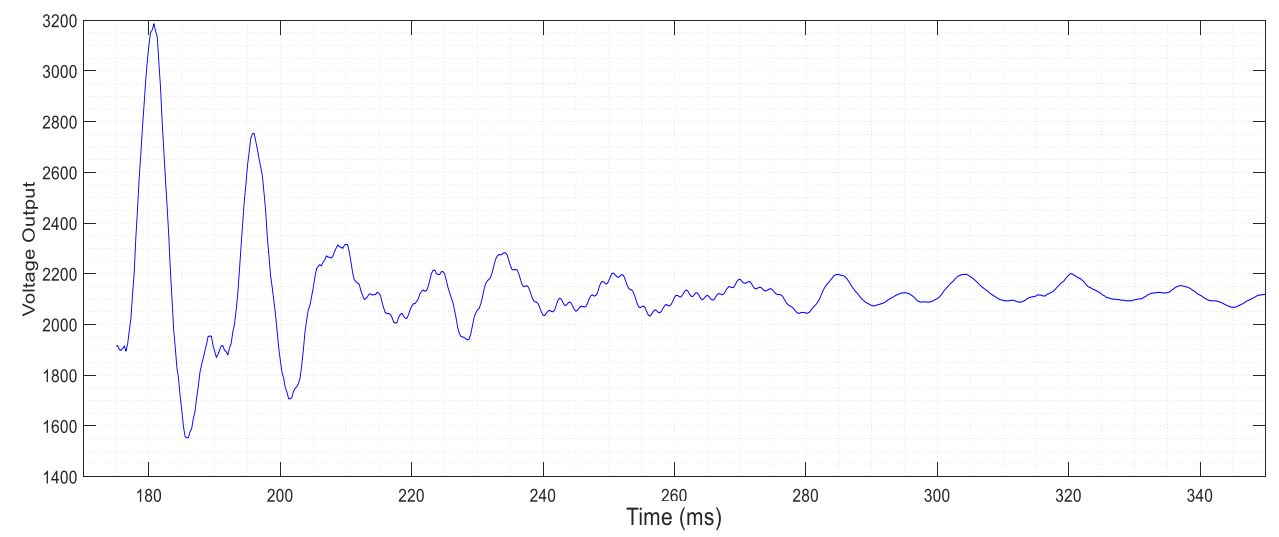

Figure (5.3): Data sampled between the laser signals for $200 \mathrm{~g}$ moving at $0.5 \mathrm{~m} / \mathrm{s}$

The sample calculations are explained below.

The steady state value has a Gaussian distribution and the first estimate or the initial value of the estimate is assumed to be the voltage datum at $174.75 \mathrm{~ms}$, i.e. $x_{o}=1916$.

Variance of the process noise model of the selected set of data was used as the initial variance of the process noise, $P_{0}=$ uncertainty of unfiltered data

The value of the standard deviation of the unfiltered data set.

For the selected set of data, the standard deviation is calculated to be Uncertainty of the measurement noise distribution, $\sigma_{v}^{2}=18.5$ (as determined in section 5.3.3)

$$
\begin{gathered}
\text { Calculate } K_{1}=\frac{P_{0}}{P_{0}+\sigma_{w}^{2}}=\frac{33.9}{33.9+18.5} \\
=0.64 \\
\hat{x}_{t+1}=\hat{x}_{t}+K_{t}\left(y_{t}-\hat{x}_{t}\right) \\
\hat{x}_{t+1}=1916+0.64(1915.11-1916) \\
\hat{x}_{1}=1915.4 \\
P_{1}=\left(1-K_{1}\right) P_{0}+\sigma_{w}^{2} \\
P_{1}=(1-0.64) 33.9 \\
=12.2
\end{gathered}
$$


$P_{1}$ is then used in calculate the next update for the Kalman gain, $K_{2}$ and the calculations repeats for all the measurements.

The same calculation process was repeated for data of $200 \mathrm{~g}$ at $0.5 \mathrm{~m} / \mathrm{s}$ in unloaded conditions.

The state estimate improves through these iterations. The average of the last $100 \mathrm{~ms}$ was used as the steady state.

Compac also uses an averaging window of $100 \mathrm{~ms}$ in estimating the weight of the fruit in the current system. The same averaging window $(100 \mathrm{~ms})$ was used in the initial comparison between the outputs of the Kalman filter with that of the existing Butterworth low pass filter. The results obtained are listed in the table below and compared with the outputs of the Butterworth filter.

Halimic \& Balachandran (1995), uses a quality factor measure the performance of the filter as below.

$$
\frac{1}{Q}=\frac{\text { standard deviation of the filtered signal }}{\text { Mean value of the filtered signal }}
$$

The performance of proposed Kalman filter was compared to that of the $5^{\text {th }}$ order Butterworth filter using the same quality factor.

A lower standard deviation for a given mean value will result in higher values for $Q$ which indicates that the variables are closer to the mean or the expected value. Therefore, the higher $Q$ indicates better filter performance.

\begin{tabular}{|l|c|c|c|c|}
\hline \multirow{2}{*}{} & \multicolumn{2}{|l|}{$5^{\text {th }}$ Order Butterworth } & Kalman filter \\
\cline { 2 - 5 } & No-load & Loaded & No -load & Loaded \\
\hline Mean & 1129.4 & 1562.75 & 1953.3 & 2353.9 \\
\hline Variance & 17.5 & 96.12 & 0.8 & 2.99 \\
\hline $\begin{array}{l}\text { \% Variation about } \\
\text { the mean }\end{array}$ & $1.4 \%$ & $2.3 \%$ & $0.16 \%$ & $0.33 \%$ \\
\hline Plateau height & - & 432 & - & 400.6 \\
\hline Q & 270.34 & 159.39 & 2186 & 1231.5 \\
\hline Settling time & - & $105 \mathrm{~ms}$ & - & $26 \mathrm{~ms}$ \\
\hline
\end{tabular}

Table 5.1: Results comparison for $200 \mathrm{~g}$ at $0.5 \mathrm{~m} / \mathrm{s}$ during $100 \mathrm{~ms}$ period at the end of the signal time for load cell 1 . 


\begin{tabular}{|l|c|c|c|c|}
\hline \multirow{2}{*}{} & \multicolumn{2}{|l|}{$5^{\text {th }}$ Order Butterworth } & \multicolumn{2}{l|}{ Kalman filter } \\
\cline { 2 - 5 } & No-load & Loaded & No -load & Loaded \\
\hline Mean & 1033.9 & 1419.7 & 1676.9 & 2130.2 \\
\hline Variance & 8.1 & 34.2 & 5.4 & 8.81 \\
\hline $\begin{array}{l}\text { \% Variation about } \\
\text { the mean }\end{array}$ & $1.6 \%$ & $1.8 \%$ & $0.55 \%$ & $0.33 \%$ \\
\hline Plateau height & - & 385 & - & 453.3 \\
\hline Q & 363.4 & 242.7 & 720.7 & 793.3 \\
\hline Settling time & - & $100 \mathrm{~ms}$ & - & $50 \mathrm{~ms}$ \\
\hline
\end{tabular}

Table 5.2: Results comparison for $200 \mathrm{~g}$ at $0.5 \mathrm{~m} / \mathrm{s}$ during $100 \mathrm{~ms}$ period at the end the signal time for load cell 2.

The comparison showed in the table 5.1 and 5.2 shows that the Kalman filter outperforms the Butterworth low pass filter in many aspects.

- It reduces the settling time from $105 \mathrm{~ms}$ to $26 \mathrm{~ms}$ in loadcell 1 and $100 \mathrm{~ms}$ to $50 \mathrm{~ms}$ in load cell 2 . This enable the transient response to reach the steady state faster resulting in larger 'averaging window'.

- The variance, and therefore the standard deviation, has a lower value when compared with that of the Butterworth filter. This reduces the $95 \%$ confidence interval reducing the margin of error of the filtered signal.

- The quality factor which measures the filter performance has increased implying better filter performance in this application.

However, the following differences between the two filters were noted.

- Butterworth low pass filter attenuates the high frequency components of the signal throughout the signal time.

- In the Kalman filter, the state converges towards the steady state towards the end of the signal time. It also provides attenuation of the high frequency components of the signal as the signal converges towards the steady state. A suitable averaging window of $50 \%$ of the signal time was selected after examining all the results. 
The outputs of Kalman filter algorithm is then used to develop a calibration method to estimate the weight of the fruits. The results and simulations are listed and explained in chapter 6.0 . 


\section{Chapter 6.0 Results and Simulations}

\subsection{Introduction}

The Kalman filter algorithm was tested on numbers of data sets provided by Compac. The results and simulations are listed and discussed in this chapter. The results obtained from the Kalman filter were used to propose a calibration method. However, the calibration method needs to be verified using more data samples.

A set of data ('loaded' data) was sampled between the laser signals when the cup containing the fruit was on the weighing table, and was used to estimate the average value of the filtered data around the plateau. Data was collected before the cup arrived at the load cell referred to as unloaded data, was analysed separately. The difference between the averages in 'loaded data' and 'unloaded data' was the increase of the mean value of the signal (plateau height) that is used to develop a calibration method.

Results of number of tests were presented in two sections;

- Constant mass at varying speeds.

- Varying masses at constant speed.

\subsubsection{Constant mass at varying speeds}

Among the data provided by Compac, four data sets were available for $200 \mathrm{~g}$ mass at three different speeds.

$$
\begin{aligned}
& \text { - } \quad 200 \mathrm{~g} \text { at } 0.5 \mathrm{~m} / \mathrm{s} \\
& \text { - } \quad 200 \mathrm{~g} \text { at } 1.0 \mathrm{~m} / \mathrm{s} \\
& \text { - } \quad 200 \mathrm{~g} \text { at } 1.5 \mathrm{~m} / \mathrm{s}
\end{aligned}
$$




\section{Filtered signal around the plateau (Loaded data)}

The figure 6.1 shows the 'loaded data' for $200 \mathrm{~g}$ fruit moving at $0.5 \mathrm{~m} / \mathrm{s}, 1.0 \mathrm{~m} / \mathrm{s}$ and $1.5 \mathrm{~m} / \mathrm{s}$.
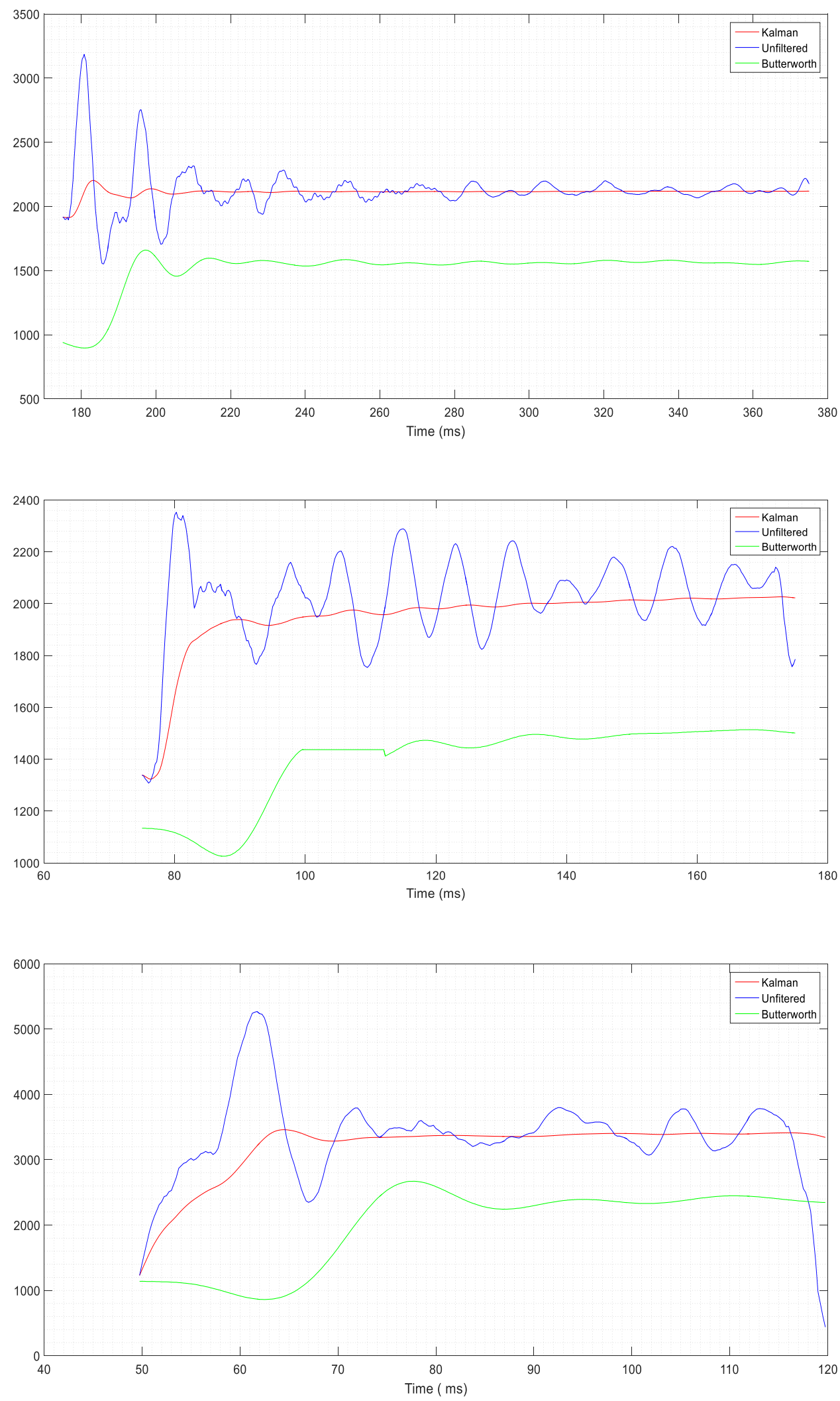

Figure (6.1): Graphs of unfiltered and filtered data of $200 \mathrm{~g}$ moving at (a) $0.5 \mathrm{~m} / \mathrm{s}$ (b) $1.0 \mathrm{~m} / \mathrm{s}$ (c) $1.5 \mathrm{~m} / \mathrm{s}$ 
The results obtained from the filtered signal using the Kalman filter algorithm is compared with that of the existing low pass filter output and the summary of the results is listed in Table 6.1 and 6.2 .

Compac uses a weighing window that is a given percentage of the full cycle in the latter part of the signal as shown in Figure (6.3). Similar weighing window of $50 \%$ of the signal time was used in obtaining the results shown in Table (6.1) and Table (6.2).

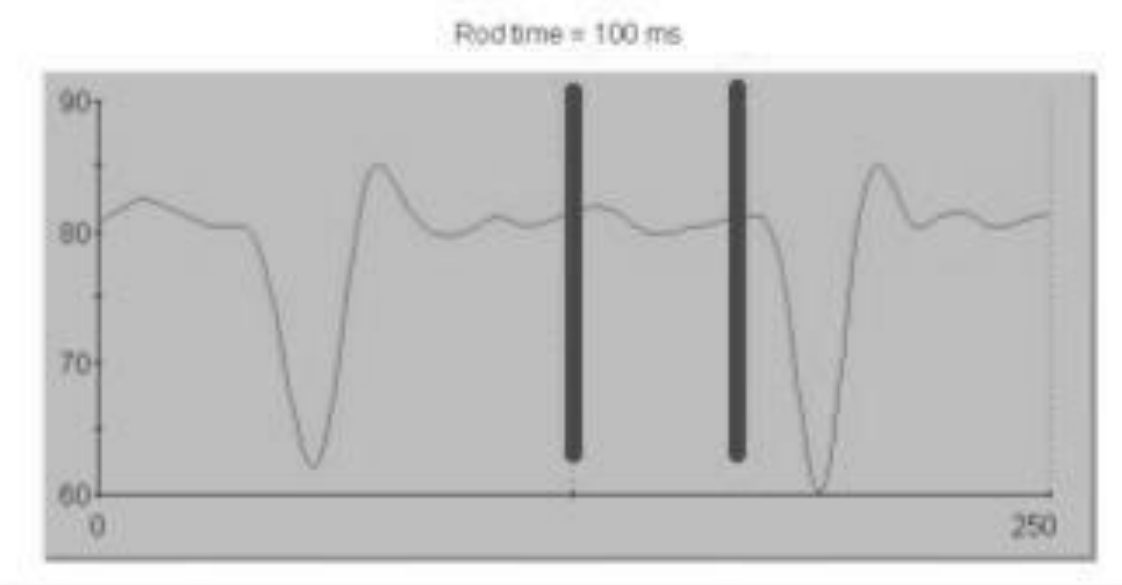

Figure (6.2): The weighing window used in the current system ('Compac Sorting Equipment', personal communication, June 2017).

\begin{tabular}{|c|c|c|c|c|c|}
\hline \multirow{2}{*}{$\begin{array}{l}\text { Conveyor } \\
\text { speed } \\
(\mathrm{m} / \mathrm{s})\end{array}$} & \multicolumn{2}{|c|}{ Butterworth } & \multicolumn{2}{|c|}{ Kalman } & \multirow{2}{*}{$\begin{array}{c}\text { \% reduction } \\
\text { (improvement) } \\
\text { in Std }\end{array}$} \\
\hline & Mean & $\operatorname{Std}^{8}$ & Mean & Std & \\
\hline 0.5 & 1419.7 & 34.1 & 2363.0 & 7.7 & 77.4 \\
\hline 1.0 & 1487.1 & & 2458.0 & 9.0 & \\
\hline 1.5 & 1703.3 & 48.5 & 2822.5 & 15.8 & 74.4 \\
\hline
\end{tabular}

Table (6.3): Comparison of 'loaded data' for $200 \mathrm{~g}$ moving at $0.5 \mathrm{~m} / \mathrm{s}, 1.0 \mathrm{~m} / \mathrm{s}$ and $1.5 \mathrm{~m} / \mathrm{s}$.

The standard deviation is a measure of variation of the set of data in the filtered signal. The reduction in the standard deviation implies lower variation of data thus increases reliability of the estimate of the mean.

\footnotetext{
${ }^{8}$ Std- Standard deviation.
} 
The improvements in the standard deviation made by the Kalman filter is above $74 \%$ for $200 \mathrm{~g}$ mass moving at all three speeds. This yields a more reliable mean value for the filtered signal in the weighing window.

As presented in Table 6.2, the mean values increase as the speed increase in both cases. The results obtained from both filters exhibit an increasing trend and the trend is not linear in both cases. The graph uses only 4 sets of data; thus the results need to be verified by more tests.

\section{Settling time}

Settling time tabulated in Table (6.2), is the time required for the system response curve to reach and stay within $2 \%$ about the mean value. The data was examined using spread sheets in MS Excel and a trial and error method was used to estimate the settling time in each case. When the system parameters are known, the settling time can be calculated using the damping ratio and the natural frequency of the system (equation (3.)).

\begin{tabular}{|c|c|c|c|c|}
\hline \multirow{2}{*}{$\begin{array}{c}\text { Conveyor } \\
\text { speed } \\
(\mathrm{m} / \mathrm{s})\end{array}$} & \multicolumn{2}{|c|}{ Settling time (LC1) } & \multicolumn{2}{c|}{ Settling time (LC2) } \\
\cline { 2 - 5 } & Butterworth & Kalman & Butterworth & Kalman \\
\hline 0.5 & 112 & 28 & 100 & 31 \\
\hline 1.0 & 67 & 47 & 63 & 17 \\
\hline 1.5 & - & 27 & - & 32 \\
\hline
\end{tabular}

Table (6.2): Comparison of settling times for $200 \mathrm{~g}$ moving at $0.5 \mathrm{~m} / \mathrm{s}, 1.0 \mathrm{~m} / \mathrm{s}$ and $1.5 \mathrm{~m} / \mathrm{s}$.

In summary, the Kalman filter shows significant improvement in steady state signal and the settling time for lower speeds, i.e. $0.5 \mathrm{~m} / \mathrm{s}$ and $1.0 \mathrm{~m} / \mathrm{s}$ for $200 \mathrm{~g}$ mass.

At $1.5 \mathrm{~m} / \mathrm{s}$, the existing filtered signal does not reach a state where the error margin is $2 \%$ or lower. With the use of the Kalman filter, the system response reaches and stays within $2 \%$ of the mean value in $32 \mathrm{~ms}$ which is approximately $50 \%$ of the signal time. This leaves a weighing window of $28 \mathrm{~ms}$.

The signal filtered with the Butterworth filter only reaches $9.6 \%$ error margin in $32 \mathrm{~ms}$. The percentage error decreases as the time progresses during the signal time but does not reach $3 \%$ error margin. 


\section{Filtered signals before arrival at the load cell (Unloaded data)}

The test results for unloaded data for $200 \mathrm{~g}$ moving $0.5 \mathrm{~m} / \mathrm{s}, 1.0 \mathrm{~m} / \mathrm{s}$ and $1.5 \mathrm{~m} / \mathrm{s}$ are presented in this section. The figure 6.4 shows the graphs of filtered and unfiltered data in 'unloaded' conditions.

\begin{tabular}{|c|c|c|c|c|c|}
\hline \multirow{2}{*}{$\begin{array}{c}\text { Conveyor } \\
\text { speed } \\
(\mathrm{m} / \mathrm{s})\end{array}$} & \multicolumn{2}{|c|}{ Butterworth } & \multicolumn{2}{|c|}{ Kalman } & \multirow{2}{*}{$\begin{array}{c}\% \text { reduction } \\
\text { (improvement) } \\
\text { in } \mathrm{Std}\end{array}$} \\
\hline & Mean & Std & Mean & Std & \\
\hline 0.5 & 1033.8 & 8.1 & 1953.3 & 0.8 & 90.1 \\
\hline 1.0 & 1031.8 & 44.7 & 1959.5 & & \\
\hline 1.5 & 1031.8 & 24.6 & 1952.5 & 5.7 & 76.8 \\
\hline
\end{tabular}

Table (6.3): Comparison of unloaded data for $200 \mathrm{~g}$ moving at $0.5 \mathrm{~m} / \mathrm{s}, 1.0 \mathrm{~m} / \mathrm{s}$ and $1.5 \mathrm{~m} / \mathrm{s}$.

The summary of results presented in Table (6.3) for 'unloaded data' shows that the overall mean values do not increase with the increase of speed for unloaded data in both cases. The mean values obtained from the Butterworth filter and Kalman filter are approximately 1033 and around 1953, respectively.

Similar to the performance in 'loaded data', the Kalman filter shows significant improvements in standard deviation in filtering 'unloaded data'.

\subsubsection{Varying masses at a constant speed.}

The data for two masses moving at the same speed have been used to study the characteristics of variation of mass at a given conveyor speed. In this analysis, six sets of data have been used as below.

- $\quad 200 \mathrm{~g}$ at $0.5 \mathrm{~m} / \mathrm{s}$ ( one set of data)

- $\quad 573.1 \mathrm{~g}$ at $0.5 \mathrm{~m} / \mathrm{s}$ ( five sets of data)

The mean value of the signal has increased as the mass increases from $200 \mathrm{~g}$ to $573.1 \mathrm{~g}$.

The increasing trend of the mean value was an expected result that validates the expected behaviour of the load cell under the application of load.

The signal time is $185 \mathrm{~ms}$ at the conveyor speed of $0.5 \mathrm{~m} / \mathrm{s}$ for the selected data set. The settling time (time to reach $2 \%$ error margin) is in the range of $170 \mathrm{~ms}$ for heavier fruits. The 
results tabulated in the Table (6.4) show a weighing window less than $15 \mathrm{~ms}$ for most of the data sets.

The Kalman filter improves the settling time by reaching the steady state approximately in $40 \mathrm{~ms}$, leaving a weighing window of about $145 \mathrm{~ms}$. This is approximately $75 \%$ of the signal time.

Similar to the results in the two previous comparisons, significant improvement in the standard deviation is observed when using the Kalman filter.

\begin{tabular}{|c|c|c|c|c|c|c|}
\hline \multirow{2}{*}{ Mass (g) } & \multicolumn{3}{|c|}{ Butterworth } & \multicolumn{3}{c|}{ Kalman } \\
\cline { 2 - 7 } & Mean & Std & $\begin{array}{c}\text { Settling } \\
\text { time (ms) }\end{array}$ & Mean & Std & $\begin{array}{c}\text { Settling } \\
\text { time (ms) }\end{array}$ \\
\hline 200.0 & 1562.8 & 9.8 & 112 & 2130.2 & 1.7 & 36 \\
\hline 573.1 & 2323.1 & 43.9 & 172 & 2874.1 & 9.0 & 45 \\
\hline 573.1 & 2363.0 & 36.7 & 158 & 2924.9 & 5.0 & 30 \\
\hline 573.1 & 2343.9 & 68.9 & - & 2891.9 & 11.1 & 40 \\
\hline 573.1 & 2286.5 & 35.9 & 174 & 2846.4 & 4.0 & 35 \\
\hline 573.1 & 2315.1 & 52.8 & 174 & 2884.8 & 6.3 & 35 \\
\hline
\end{tabular}

Table (6.4): Comparison of 'loaded data' for $200 \mathrm{~g}$ and $573.1 \mathrm{~g}$ moving at $0.5 \mathrm{~m} / \mathrm{s}$

\section{Single carrier}

Data provided by Compac shows the weigh graph of a single cup moving over the weighing table. There are two clear regions where 'loaded' and 'unloaded' data can be sampled for analysis.

The sudden change in signal value, (that was referred to as the 'plateau height' in this thesis), was estimated by calculating the difference between the average values of 'loaded data' and 'unloaded data'. 

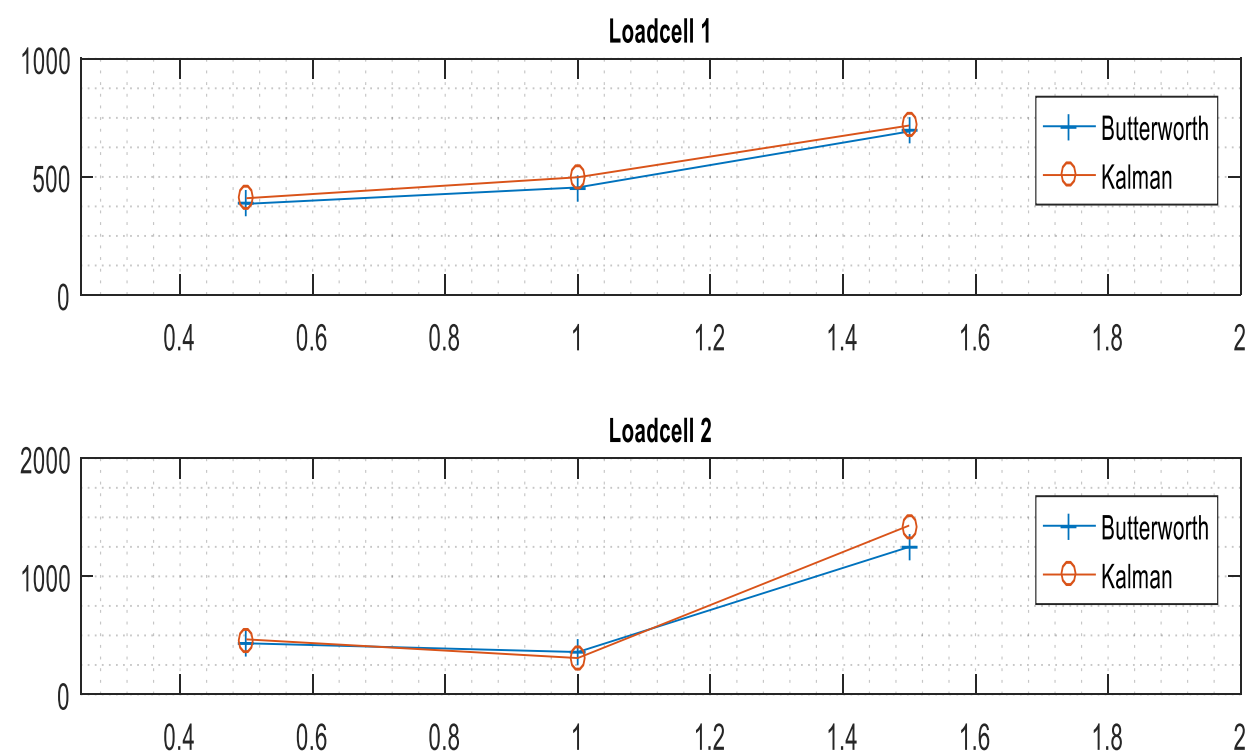

Figure (6.5): variation of the plateau height with the increase of conveyor speed
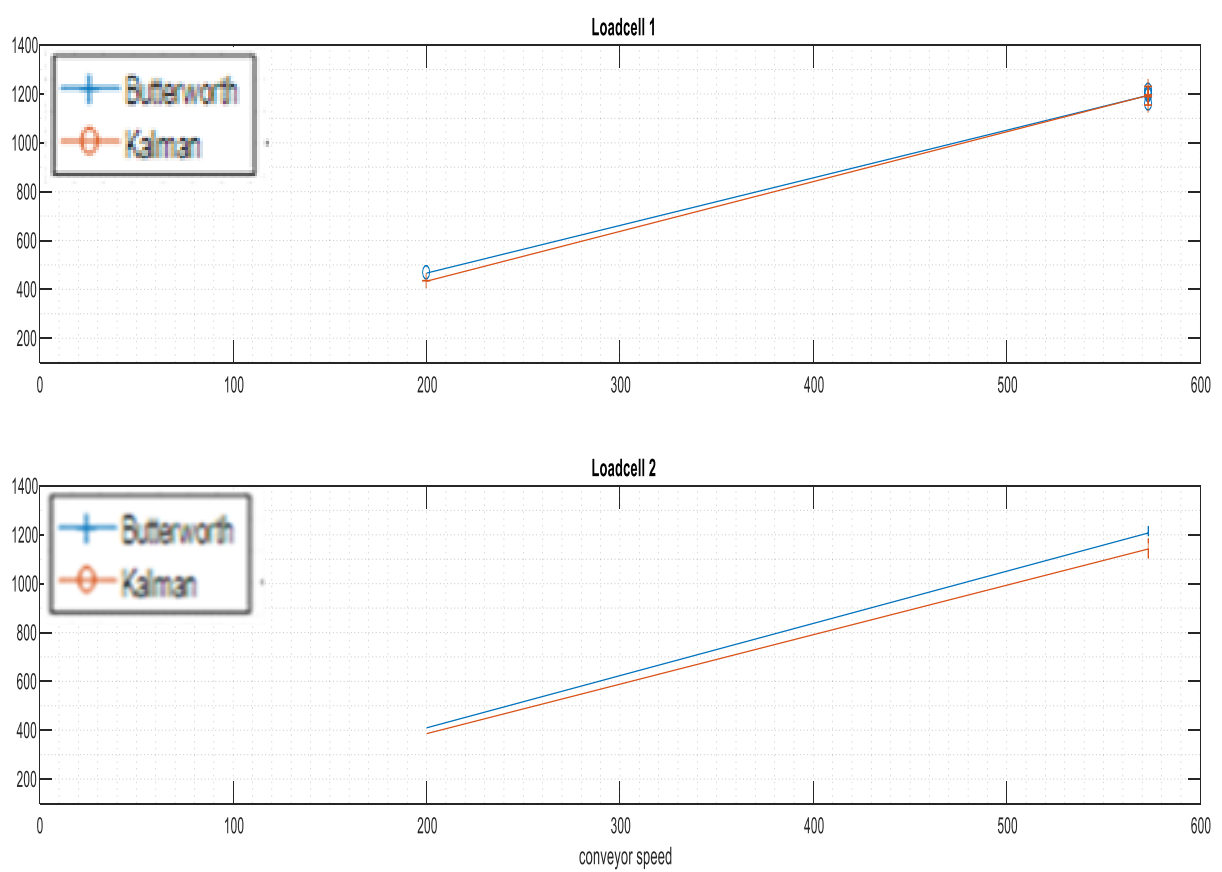

Figure (6.6): variation of the plateau height with the increase of mass

Observed inthese graphs are:

- Both Butterworth filter and the Kalman filter response yield similar variation as the speed increases. The gap between the average plateau heights has increased at the highest speed available, i.e. $1.5 \mathrm{~m} / \mathrm{s}$. 
- The variation of the plateau height with the increase of mass shows similar trends. However, the graph uses the information of two masses, i.e. $200 \mathrm{~g}$ and $573.1 \mathrm{~g}$.

\section{Multiple carriers}

Figure (6.2) (provided in the initial presentation made by Compac) shows a typical weigh graph of multiple carriers.

Visualised in the graph, sampling of 'loaded data' is possible, however, 'unloaded' region is not available for data sampling in real time.

The sudden increase of signal value can be calculated by obtaining the difference between the average signal value around the plateau and a pre-calculated reference value.

Therefore, both plateau height and the averages of weighing windows values are calculated and plotted for comparison.
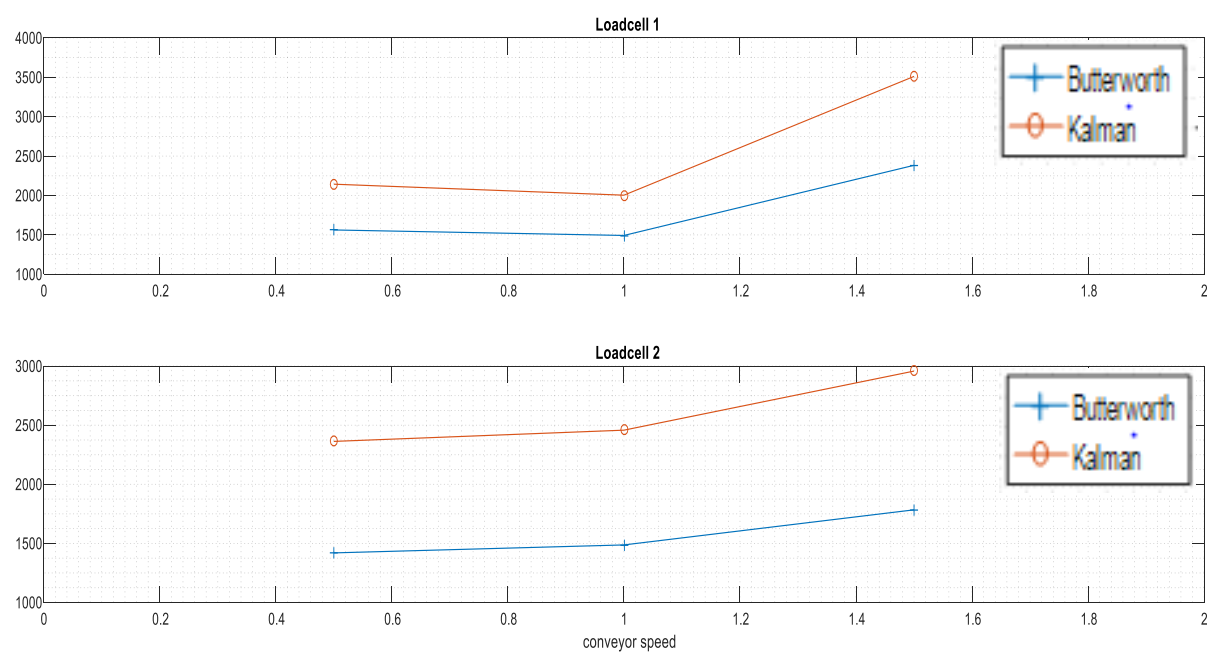

Figure (6.7) Variation of the Average signal value (loaded) with the increase of the conveyor speed. 

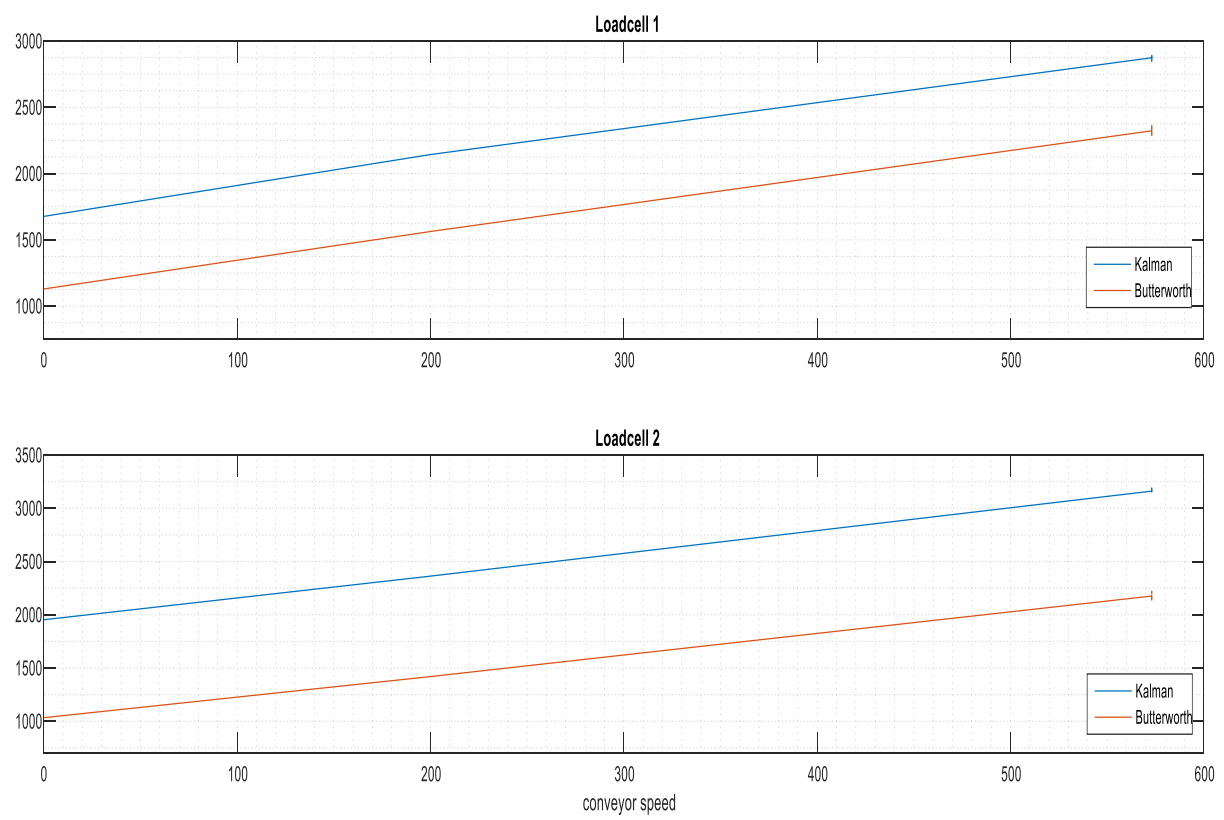

Figure (6.8) Variation of the Average signal value (loaded) with the increase of mass.

Observed in these graphs are:

- The variation of plateau heights calculated using the Kalman filter shows some deviation from the output of the Butterworth filter at higher speeds.

- A very similar increasing trend is observed in the 'Average signal value' vs 'mass' which was drawn using data for two masses.

The results will be discussed in Chapter 7.0.

\subsubsection{Proposed calibration method}

The observations explained in section 6.2.1 and 6.2.2 shows that the mean value of the filtered signal increases with the increase of the mass as well as the increase of speed.

Therefore, the estimation of is of the form shown in equation (6.1).

Mass estimation, $m(e s t)=\alpha(\Delta v)+\beta(\Delta v)$;

where,

$\Delta v=$ the mean voltage output at a given time - reference voltage

$\alpha=$ the coefficient based on the change of speed from a reference value. (e.g. the gradient of the graph of increase of plateau height vs conveyor speed, assuming that the graph is linear.)

$\beta=$ the coefficient based on the change of the mass. 
However, the variation stated above may be not be linear.

The proposed calibration method is to use a look up table (LUT).

A lookup table is an array that contains multidimensional data. The numerical data stored in the array are either pre-calculated or experimental data. It provides means mapping inputs to outputs using an array indexing method.

It allows a digital processor to calculate a value of a function without performing complex, advanced mathematical operations. 


\section{Chapter 7.0 \\ Conclusion}

\subsection{Introduction}

A one-dimensional Kalman filtering technique has been explored as a possible solution that will enable improved accuracy of dynamic weighing.

The dynamic behaviour of the weighing mechanism was studied and analysed using a mathematical model: a second order differential equation.

The step response of the second order differential equation is given by the equation (3.5),

$$
\begin{aligned}
& x(t)=\frac{m g}{k}-\frac{m g}{k} \frac{\left(2 \xi \omega_{n}+1\right)}{\omega \sqrt{1-\xi^{2}}} e^{-\xi \omega t \sqrt{1-\xi^{2}}} \\
& x(t)=\text { steady state response }+ \text { transient response }
\end{aligned}
$$

It consists of a constant state (or a steady state) of magnitude $\frac{m g}{k}$ and a decaying oscillatory component. The constant state, or the steady state, that is responsible for the weight of the fruit was estimated using the 1-dimensional Kalman filter algorithm. A simplified step response of the system was considered as two sets of random data. The difference between the steady state of each set of data was used to estimate the mass of the fruit being moved over the weighing table.

A calibration method was the proposed to estimate the mass. The results obtained are discussed in section 7.2.

\subsection{Results}

It is given that the Butterworth filter yields satisfactory results at speeds lower than 1.0 $\mathrm{m} / \mathrm{s}$ and masses less than $250 \mathrm{~g}$. The results obtained from the Kalman filter show similar behaviour to that of Butterworth filter at lower speeds (Figure (6.7)). Some variations 
are observed at the higher speeds available, i.e. $1.5 \mathrm{~m} / \mathrm{s}$. These results need to be verified by more data samples.

\section{Standard deviation}

The standard deviation of the filtered data which quantifies the variation or the dispersion of data about its mean value was one of the tools used to compare the outcome of the Butterworth filter and the Kalman filter. The constancy of the mean values also taken into account when comparing the two filtered signals using the standerd deviation. A significant reduction in the standard deviation was observed at all the speeds and the masses when using the kalman filter.

\section{Settling time}

Reducing the settilng time of the filtered signal was one of the key requirements of this project. The settling times were estimated monitoring the percentage error and the constancy of the mean value of the filtered voltage signal.

The settiling times of the filtered signal were also compared to study the improvements made be the Kalman filter.

Significant improvements were observed in setting times when the Kalman filter was used, e.g. the signal did not reach the steady state ( $2 \%$ percentage error margin) at 1.5 $\mathrm{m} / \mathrm{s}$ but reaches the steady state in $27 \mathrm{~ms}$ when the kalman filter was used.

These improvements observed in standard deviation and the settling time have made some changes to the plateau heights when compared with that of the existing values. The percentage changes are shown in table 7.1 and 7.2 below.

Observed in Table 7.1 and Table 7.2, the changes in the plateau heights are within $10 \%$ for lower speeds, i.e. $200 \mathrm{~g}$ moving at $0.5 \mathrm{~m} / \mathrm{s}$ and $573.1 \mathrm{~g}$ moving at $0.5 \mathrm{~m} / \mathrm{s}$ for both load cells. The plateau heights obtained using the Kalman filter at $1.5 \mathrm{~m} / \mathrm{s}$ deviates from that of the Butterworth conditined filter by $37.1 \%$ for load cell 1 and $23.0 \%$ for load cell 2. The increase in percentage deviation needs to be verified by more data samples and 
should be investigated futher to see if the change causes any improvements in the weight estimation at higher speeds.

\begin{tabular}{|c|l|l|c|l|l|c|}
\hline \multirow{2}{*}{$\begin{array}{c}\text { Speed } \\
(\mathrm{m} / \mathrm{s})\end{array}$} & \multicolumn{3}{|c|}{ Load cell 1 } & \multicolumn{3}{c|}{ Load cell 2 } \\
\cline { 2 - 7 } & Butterworth & Kalman & \% Change & Butterworth & Kalman & \% Change \\
\hline $0.5 \mathrm{~m} / \mathrm{s}$ & 433.35 & 466.6 & $7.7 \%$ & 385.9 & 409.7 & $6.1 \%$ \\
\hline $1.0 \mathrm{~m} / \mathrm{s}$ & 359.3 & 308.4 & $14.1 \%$ & 498.3 & 498.5 & $9.4 \%$ \\
\hline $1.5 \mathrm{~m} / \mathrm{s}$ & 1347.6 & 1714.8 & $37.2 \%$ & 692.4 & 925.5 & $23.0 \%$ \\
\hline
\end{tabular}

Table (7.1): Comparison of plateau heights for $200 \mathrm{~g}$ moving at various speeds.

\begin{tabular}{|c|l|l|l|l|l|l|}
\hline \multirow{2}{*}{$\begin{array}{c}\text { Mass } \\
(\mathrm{g})\end{array}$} & \multicolumn{3}{|c|}{ Load cell 1 } & \multicolumn{3}{c|}{ Load cell 2 } \\
\cline { 2 - 7 } & Butterworth & Kalman & \% Change & Butterworth & Kalman & \% Change \\
\hline 573.1 & 1215.6 & 1244.9 & $2.4 \%$ & 1138.8 & 1143.8 & $0.45 \%$ \\
\hline 573.1 & 1232.6 & 1214.03 & $1.5 \%$ & 1104.2 & 1195.3 & $8.2 \%$ \\
\hline 573.1 & 1153.3 & 1161.44 & $0.7 \%$ & 1184.5 & 1234.5 & $4.2 \%$ \\
\hline 573.1 & 1186.3 & 1205.5 & $1.5 \%$ & 1165.2 & 1201.8 & $3.1 \%$ \\
\hline
\end{tabular}

Table (7.2): Comparison of plateau heights for $573.1 \mathrm{~g}$ moving at $0.5 \mathrm{~m} / \mathrm{s}$

In the existing Butterworth system, it is possible the signal exceeds the $2 \%$ percentage error margin due to the presence of oscilltions about a constant mean value, in some cases. These oscillations, however, get cancelled when calculating the average of the signal.

Calculating the average provides an effect of two cascading filters thus a smoother output signal results.

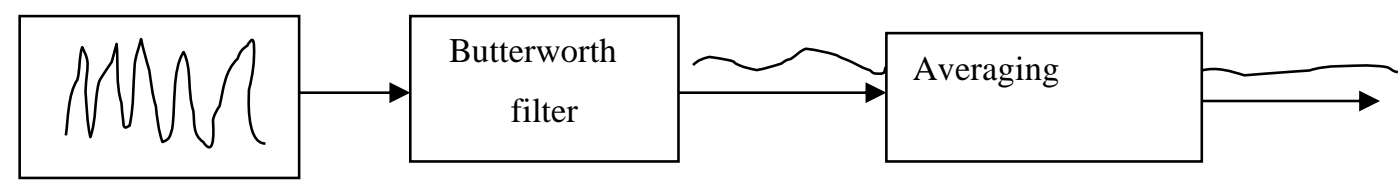

Figure (7.1): Existing Butterworth filter output. 
Averaging the filtered signal has a little benefit on the Kalman filter output that already has a stronger mean value with a lower standard deviation.

\section{Calibration method}

Information about the current calibration method is not available for comparison. However, the plateau heights vary with the changes of the masses of the fruit as well as the change of speed. A look-up-table that takes both of these variations is recommended as a calibtation technique.

\subsection{Kalman filter}

The Kalman filter was regarded as the optimal solution to data tracking and prediction problems. The filter was constructed as a mean error minimiser in the deterministic derivation. The Kalman filter has many advantages in state estimation in continuously changing systems. The system equation is in the form of a first order difference equation that uses only the information of the previous state. Hence the computation of the states is faster. The filter gain is updated every iteration of its calculations, minimising the residual with an updated gain factor in every step.

The filter is also adaptive to the data being used. All these features results in a fast response enabling the system reaching the steady state fast. The Kalman filter was explored as a possible solution for its favourable features.

In my recent research, it is found that the continuous time Kalman filter has previously been used in weight estimation in high speed dynamic weighing system using the second order differential equation as the system model. In a different approach used in this thesis, the loadcell output signal is separated into two sets of time series data. The $1-$ dimensional Kalman filter was used to estimate the underlying steady state of each data set and the difference between the loaded and unloaded data was used in weight estimation. The method is somewhat similar to the existing method where the Butterworth filtering technique where the change in average signals value of the filtered signal is used in weight estimation. Advantages of the application of Kalman filter in two stages are;

- Inherent property of the Kalman filter algorithm of fast response. 
- Use of the Kalman filter in two stages eliminates delay introduced by the sudden change of the signal value.

The new method yields positive results in reducing the settling time of the system response. However, its effect on estimation of mass required testing with more experimental data.

\subsubsection{Stability of the Kalman filter}

The stability of the Kalman filter was briefly discussed in Chapter 5.0.

The state space model used was tested for 'observability' to ensure the convergence of the filter output for the model. The convergence of the computed variables is also a requirement to establish that the Kalman filter converge for the model used.

The convergence of the 'priori error variance' with time and the variation of the Kalman gain with time for $200 \mathrm{~g}$ at $0.5 \mathrm{~m} / \mathrm{s}$ and $1.5 \mathrm{~m} / \mathrm{s}$ are shown in the Figure (7.3).
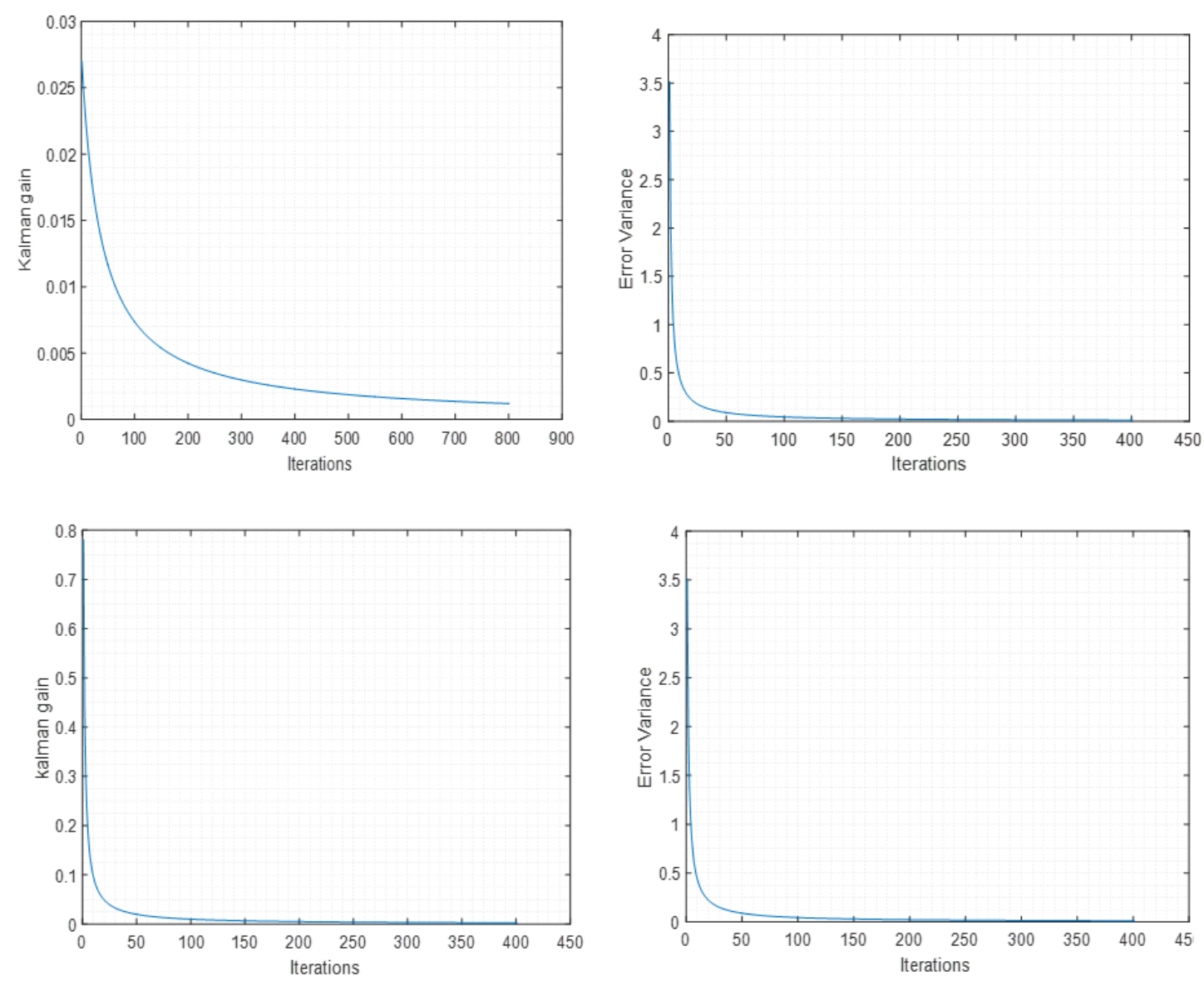

Figure (7.3): Variation of the Kalman gain with time and Error variance with time (a) Kalman gain for $200 \mathrm{~g}$ at $0.5 \mathrm{~m} / \mathrm{s}$ (b) Kalman gain for $200 \mathrm{~g}$ at $1.0 \mathrm{~m} / \mathrm{s}$ (c) Error variance for $200 \mathrm{~g}$ at $0.5 \mathrm{~m} / \mathrm{s}$ (d) Error variance for $200 \mathrm{~g}$ at $1.0 \mathrm{~m} / \mathrm{s}$ 


\subsubsection{Tuning filter parameters}

The Kalman filter, if properly tuned, gives better results than a filter that uses only model or measurement information. Proper selection of the system model and selecting error covariance $\sigma_{w}^{2}$ and $\sigma_{v}^{2}$ to exactly match the actual noise conditions will minimise the error between the prediction and the actual states (Wilson, 2016).

The determination of process noise is generally more difficult. It is used to account the mismatches between the assumed model values and true values. With the constant dynamic model used in this thesis, a perfect straight line is expected by setting the process noise variance to zero.

Determining the measurement error variance $\left(\sigma_{v}^{2}\right)$ can be done prior to the implementation of the filter by collecting samples of measurement and calculating the variance of the samples. Uncertainty of the measurements due to the intrinsic noise of the sensor is used as the measurement error variance.

The measurement uncertainty of the load cell used in the weighing system was estimated from the information obtained from load cell user guides. With zero process noise, the steady state value varies with the measurement uncertainty, $\sigma_{v}^{2}$. When the measurement uncertainty is higher, filter was slower to 'believe' the measurements.

Figure (7.2) shows the steady state values obtained when $\sigma_{v}^{2}=4.3$ and $\sigma_{v}^{2}=453$.

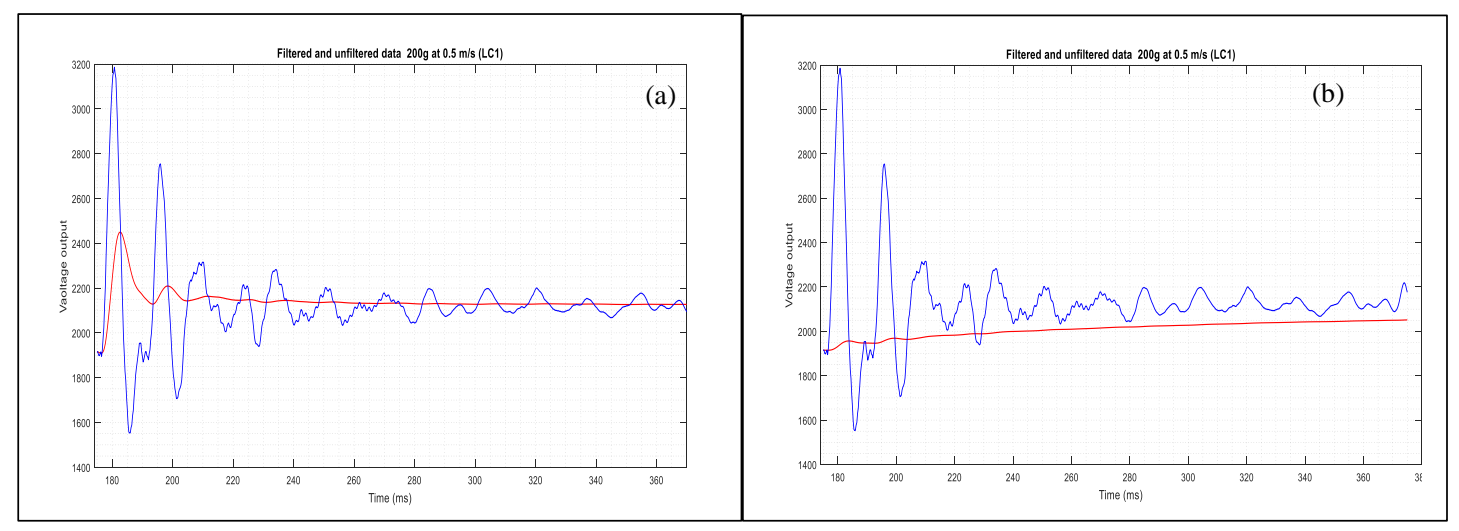

Figure (7.2): The filter response for $200 \mathrm{~g}$ at $0.5 \mathrm{~m} / \mathrm{s}$ (a) when $\sigma_{v}^{2}=4.3$ (b) $\sigma_{v}^{2}=453$

The measurement noise variance of 18.5 was used in the graphs presented in this thesis. The selected value was determined based on the information obtained from load cell user guides. 


\section{Initial estimate and initial error variance}

The initial estimate used for the simulations is the first reading in each data set in the simulations shown in thesis. The recursive algorithm begins its computations with the second reading in each data set. The alternative initial estimate tested was the average value of the sampled data set.

The choice of initial estimate did not affect the steady state value (figure (7.3)). The first reading of each data set was selected as it reduces computations as well as enables real time implementation of the algorithm.

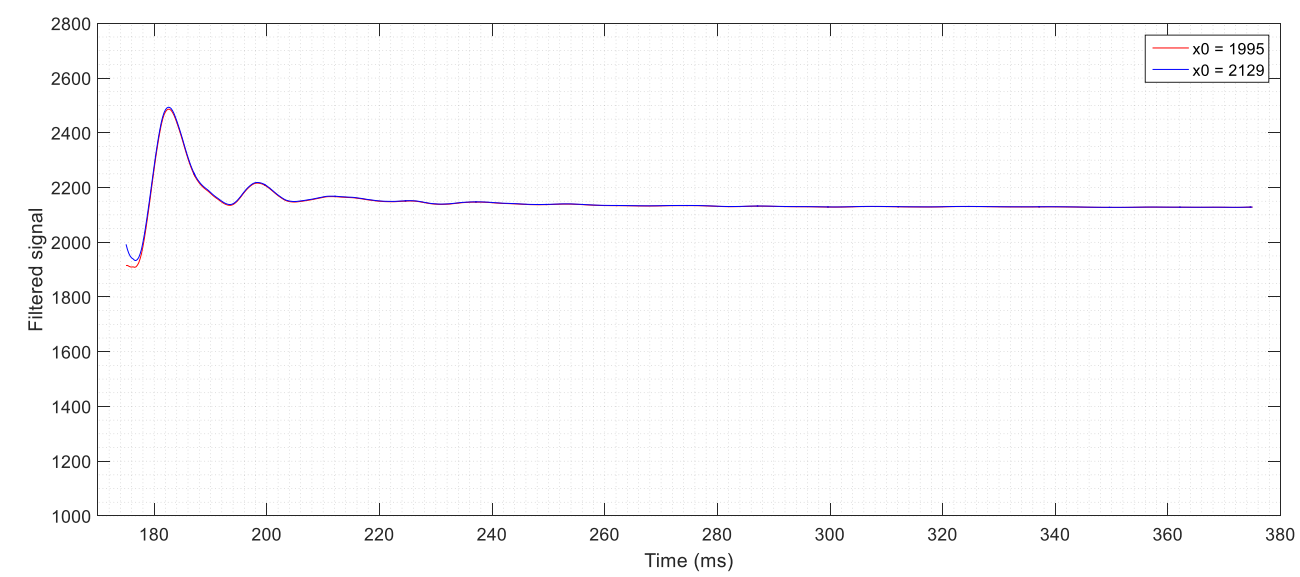

Figure (7. 3): The Kalman filter output for $200 \mathrm{~g}$ at $0.5 \mathrm{~m} / \mathrm{s}$ for two different initial estimates; $x_{0}=1995$ (the initial reading) and $x_{0}=2129$ (The average value of the sampled data)

\section{Initial error variance}

The initial error variance was the square of the difference between the two first readings in each data set. The two other error variances tested were;

- the variance of the first two readings

- the square of the difference between the first reading and the average value of the sampled data set.

All the three initial variances yield the same steady state values. There was also no effect on the settling time.

The square of the difference between the first reading and the second reading was selected as it enables real time implementation of the algorithm. It was also the most simplistic in calculations. 


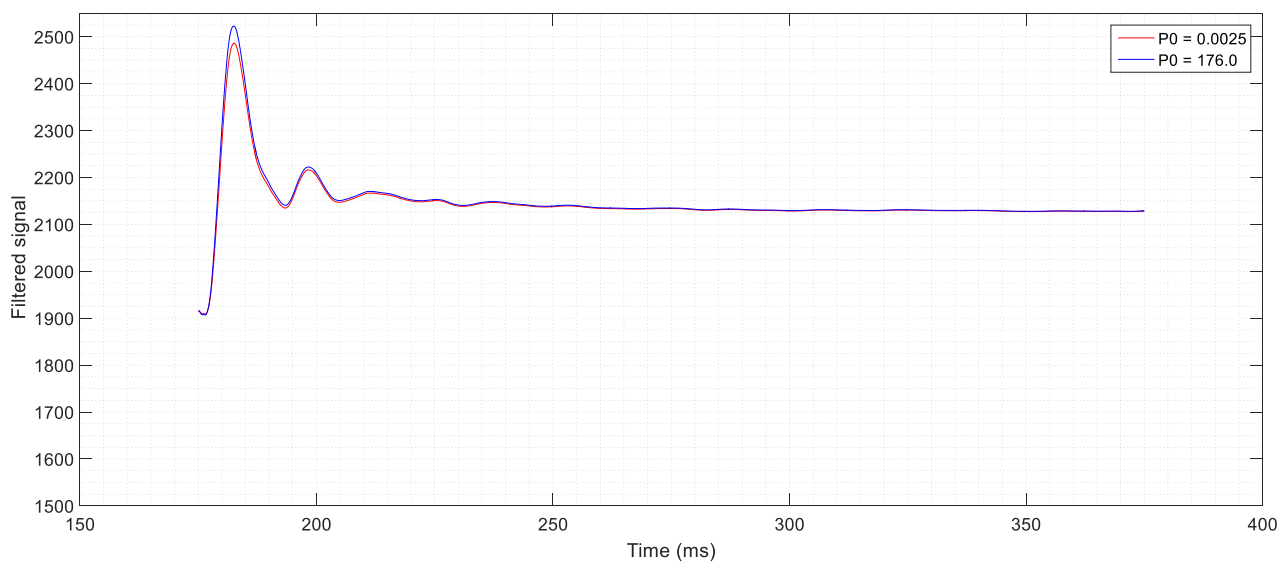

Figure (7.4): The Kalman filter output for two different initial error variance

\section{Initial measurement}

Each data set was sampled by activation of laser signals. In the former part of the plateau the signal was highly oscillatory resulting in considerable changes in the signal value within few milli-seconds. The steady state value was not affected by the stating position however the settling time could be increased depending on the position of the laser switch.

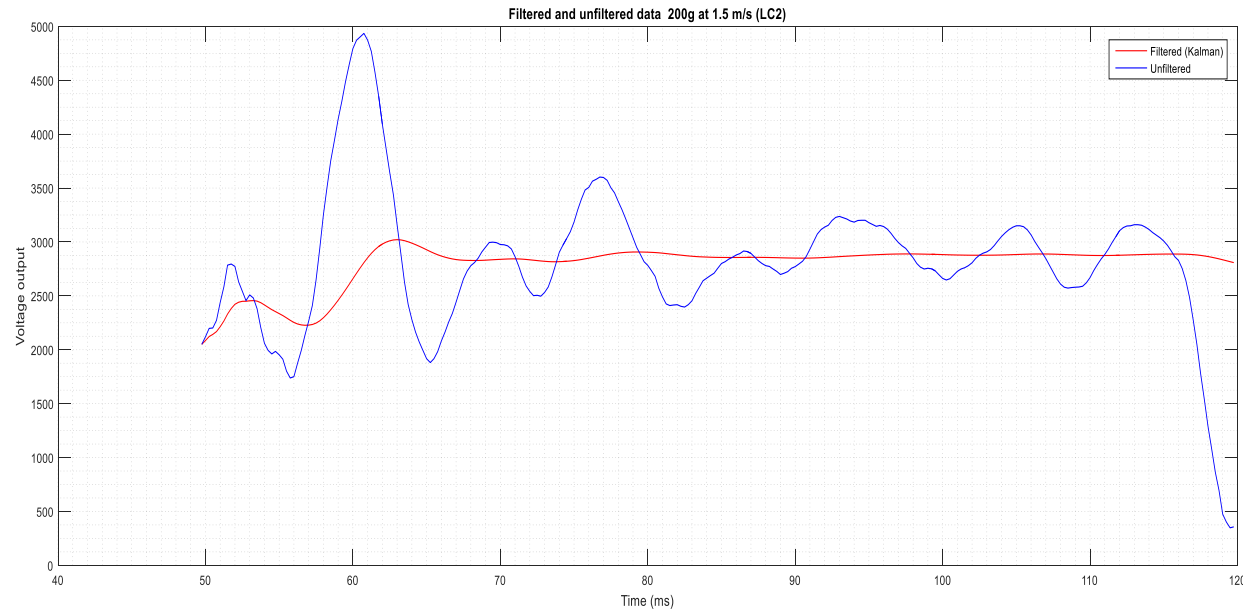

Figure (7.6): Filtered signal of $200 \mathrm{~g}$ at $1.5 \mathrm{~m} / \mathrm{s}$ for starting at $40 \mathrm{~ms}$. 


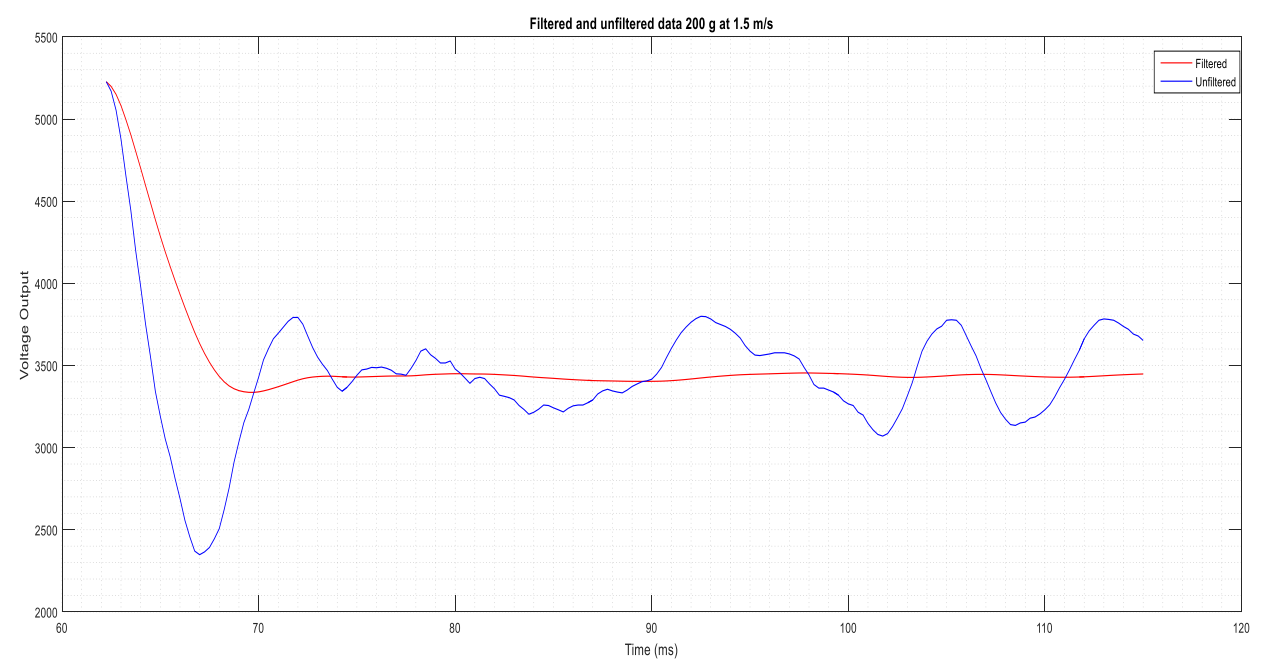

Figure (7.5): Filtered signal of $200 \mathrm{~g}$ at $1.5 \mathrm{~m} / \mathrm{s}$ starting at $62 \mathrm{~ms}$

\subsection{Fitting Vs filtering}

The existing 5torder Butterworth filter is a low pass filter that filters out the higher frequencies. The proposed Kalman filter algorithm can be considered as fitting the data into a curve specified by the state equation.

Common filtering objective of convolution based filters is to remove the signals of unwanted frequencies, (e.g. low pass filters) i.e. in low pass filtering a number of frequency components are retained in the output signal relatively unchanged.

Curve fitting (polynomial fitting) does suppress the abrupt changes and smoothed out the signal. Curve fitting alters both high frequency components as well as the low frequency of components of a signal resulting in an output function of multiple frequencies.

\subsection{Future recommendation}

In the proposed method, the weight estimation is done in three steps; estimating the 'noload steady state' estimating the 'loaded steady state' and calculating the difference to estimate the weight through calibration. A constant state model was used in two stages to obtain the steady states. Research can be carried out to study the possibilities of using a piece-wise function as the system model.

Further studies can also be carried out in using the steady state Kalman filter which is implemented for time invariant systems when the noise variances are also time invariant. 
A new system model with different noise models can be considered when using the steady state Kalman filter.

The value of the Kalman gain is updated through the recursive implementation of the filter algorithm. Therefore the Kalman gain is also time-varying. As observed in figure (7.3), the Kalman gain converges to a constant value as the system reaches the steady state. It is also seen that the error covariance converges towards a constant value as the signal reaches the steady state. The constant value of the Kalman gain is called the steady state Kalman gain (Wilson, 2016).

The steady state error covariance is determined by solving for $P_{k}$ in the Riccati equation (Wilson, 2016) and then using this constant error variance to calculate the constant Kalman gain.

$$
P_{k}=\sigma_{w}^{2}+A P_{k}-A P_{k}\left(\sigma_{w}^{2}+C P_{k}\right)^{-1} C P_{k} A
$$

The equation can be solved using the MATLAB function dare.

This is a computationally efficient procedure for calculating the steady state Kalman gain.

\subsection{Summary}

It was given that the existing Butterworth filter yields satisfactory results at lower speeds. The Kalman filter improves the filter performance in reducing the settling time of the filtered signal. This increases the stable region of the weighing window, especially at the higher speed discussed in this thesis, $1.5 \mathrm{~m} / \mathrm{s}$. Most of the data tested in this thesis are less than $1.0 \mathrm{~m} / \mathrm{s}$. There was only one set of data available at speeds higher than $1.0 \mathrm{~m} / \mathrm{s}$. However, further tests need to be carried to investigate the impact of the speed on estimation of mass.

As detailed in section 7.3, the settling time is affected by the initial reading. Some experiments would be required to position the laser switches in order to capture the best set of data for optimum results. 
Appendix A

\section{Kalman filter MATLAB code}

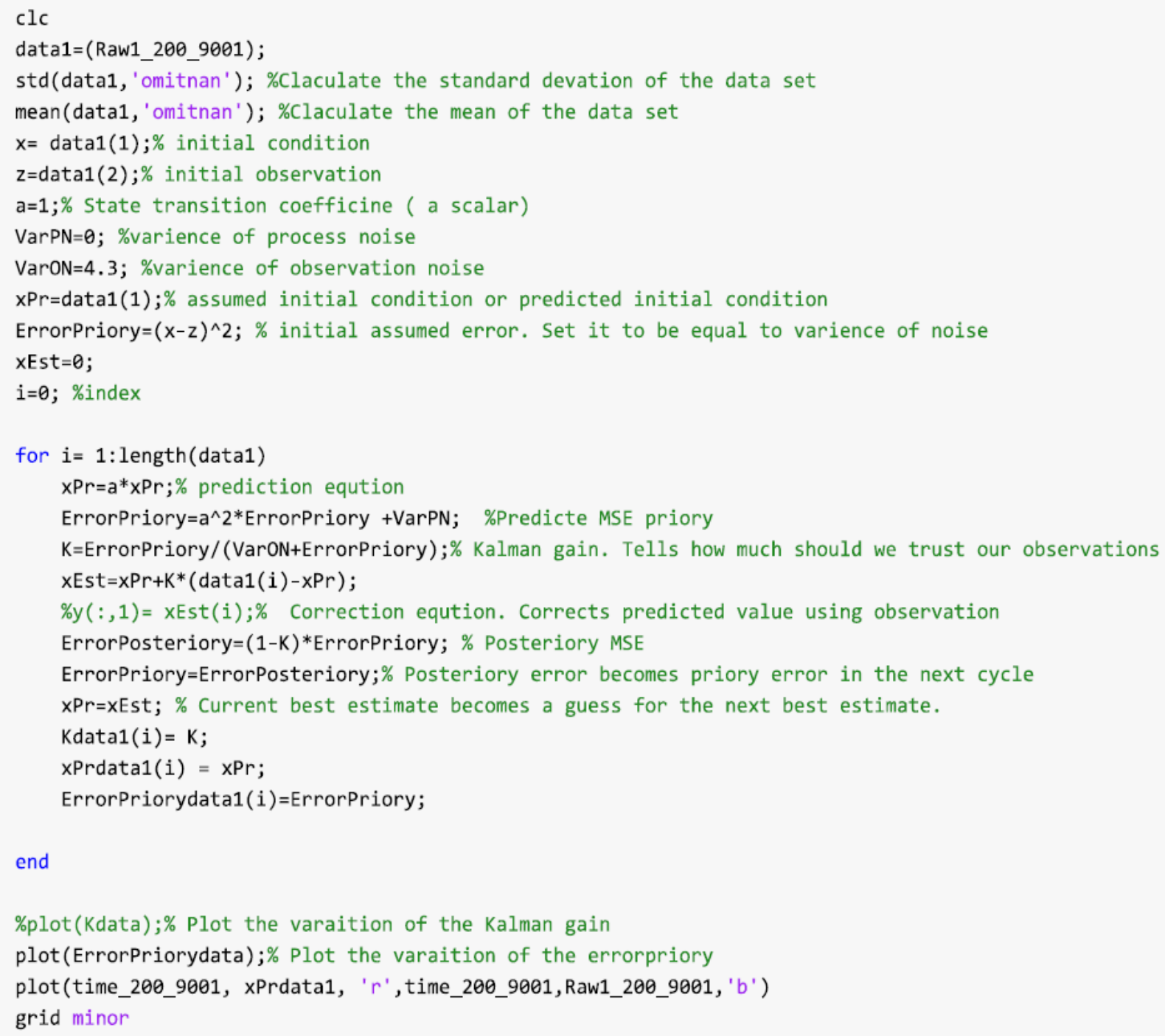


Appendix B

Graphs of Kalman filter output and stability
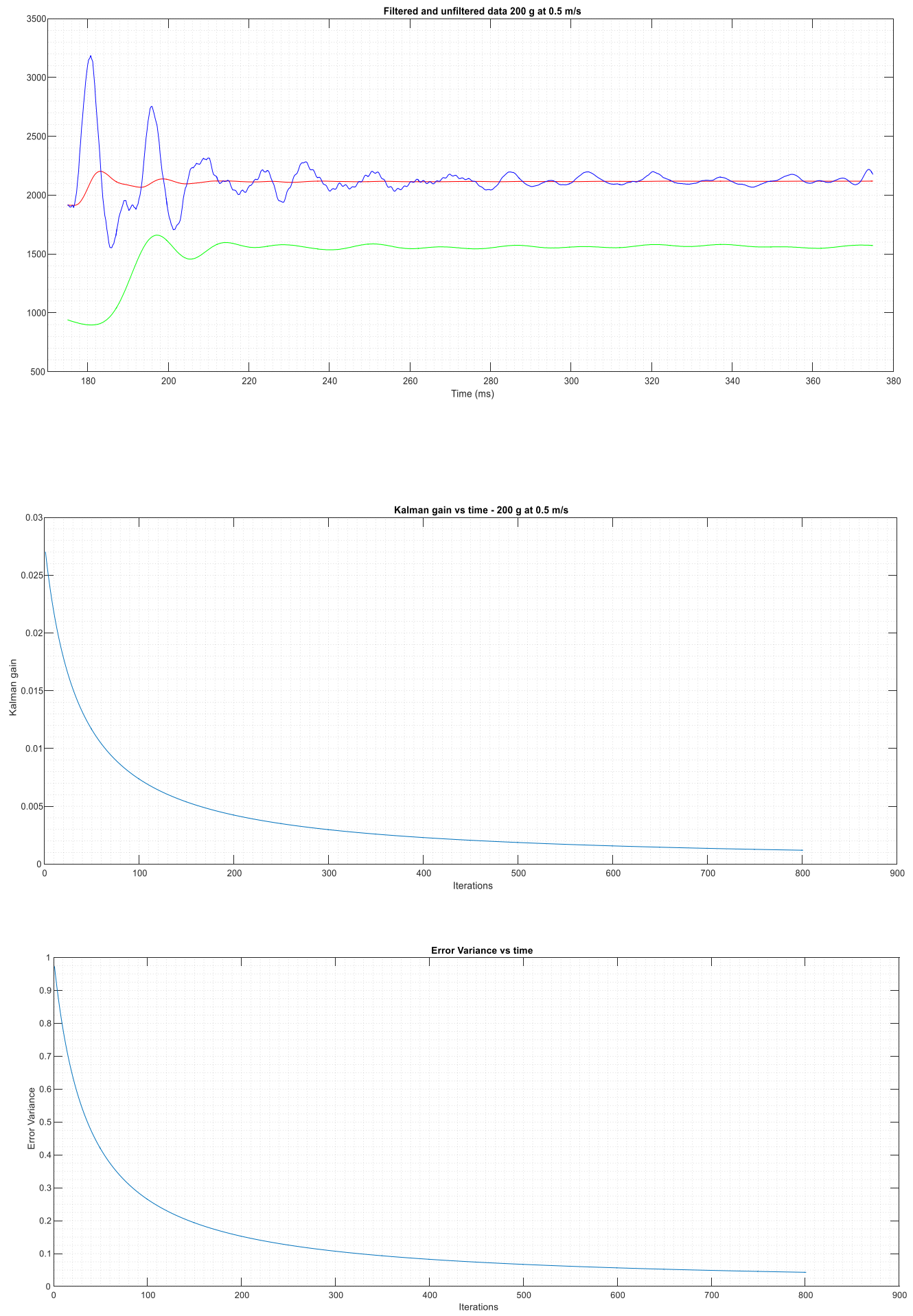

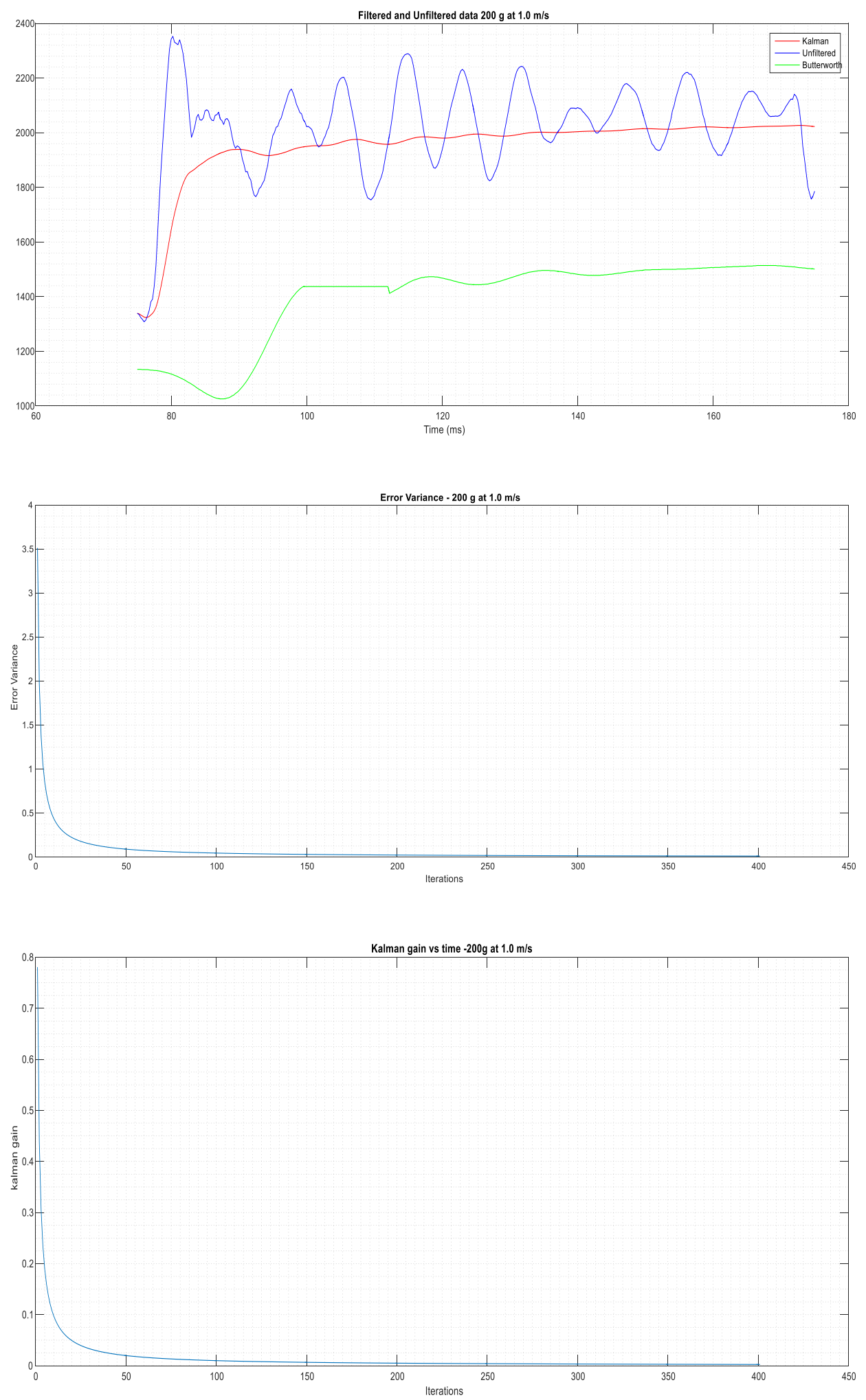

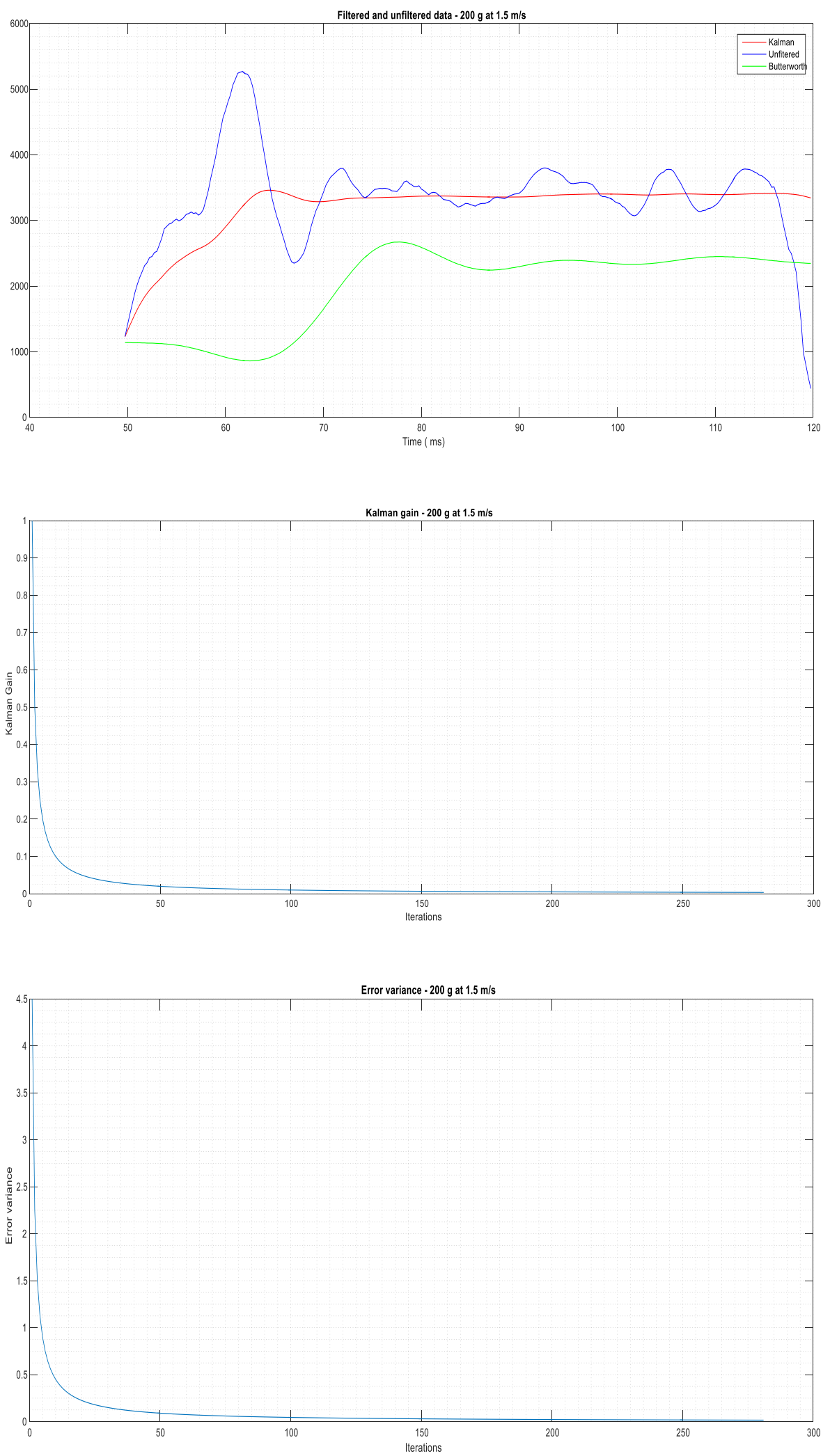

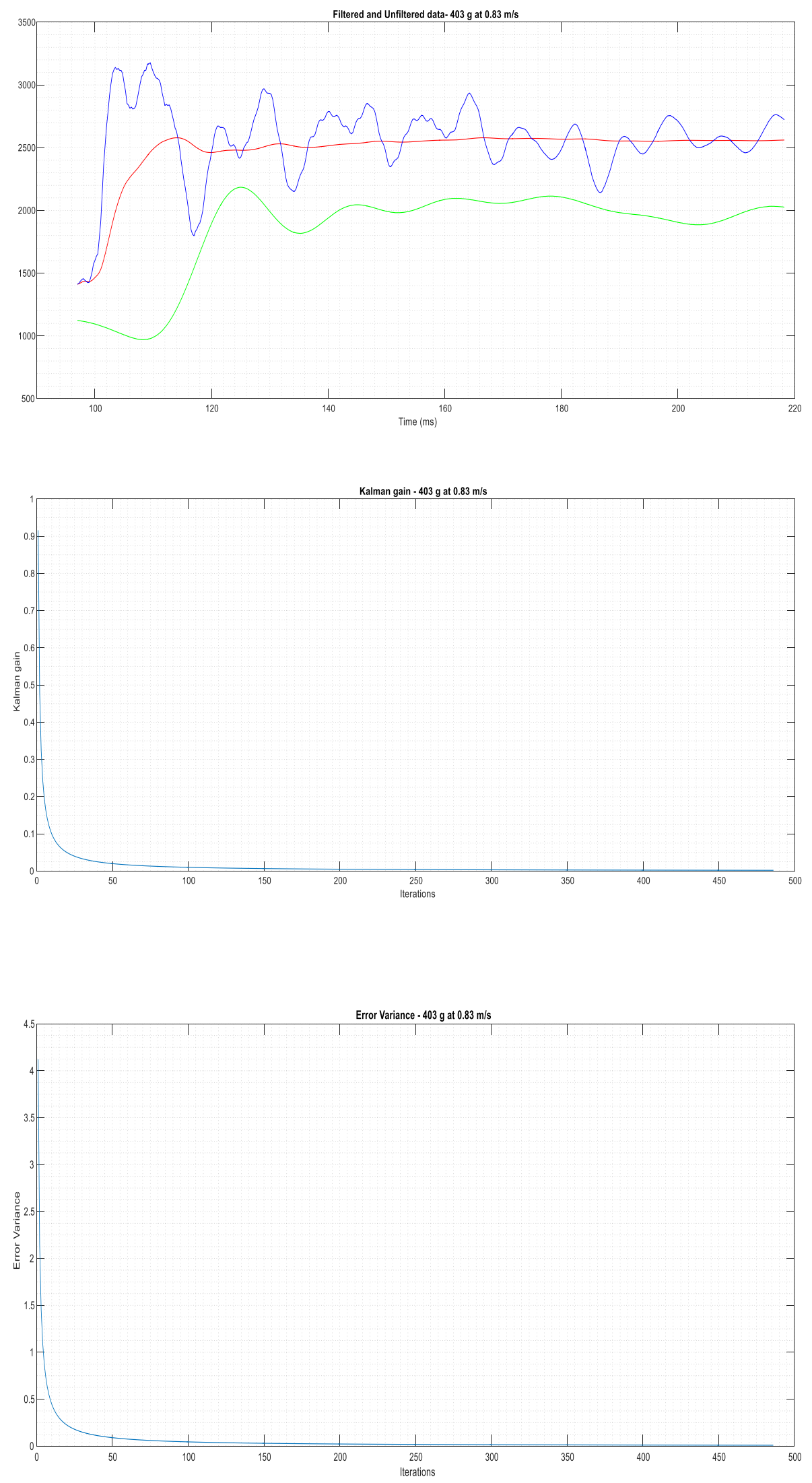

79 

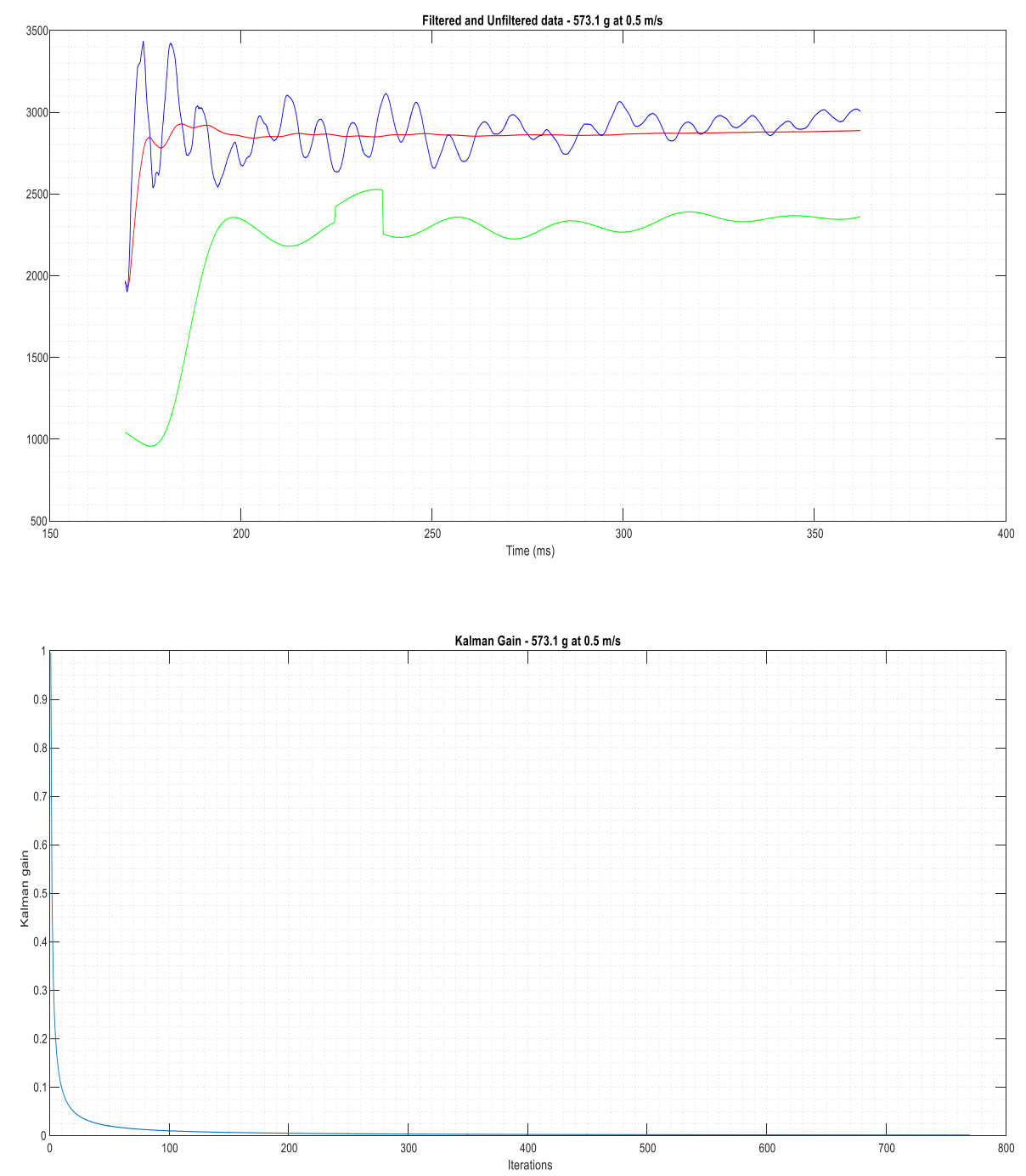
Appendix C

Power spectra analysis
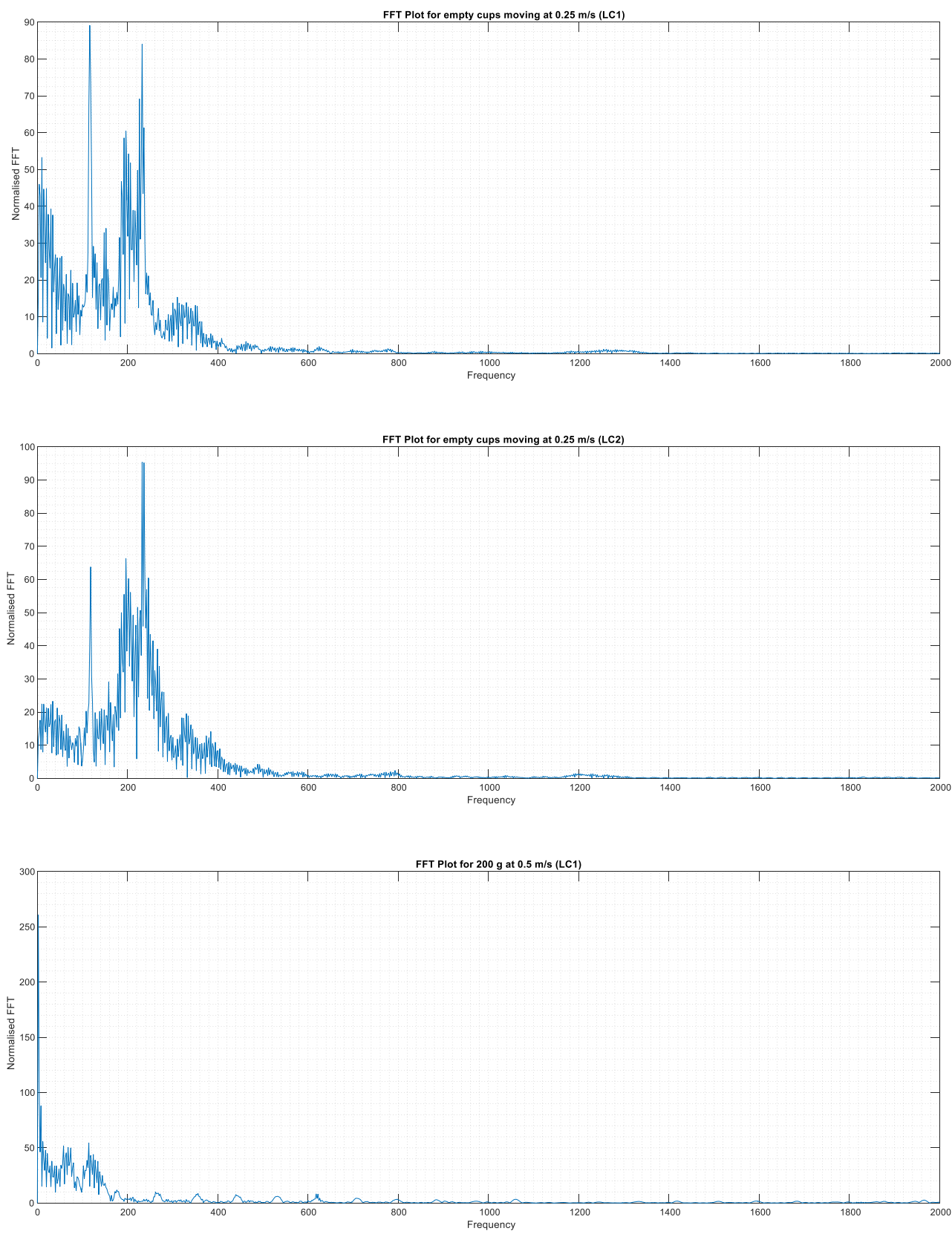

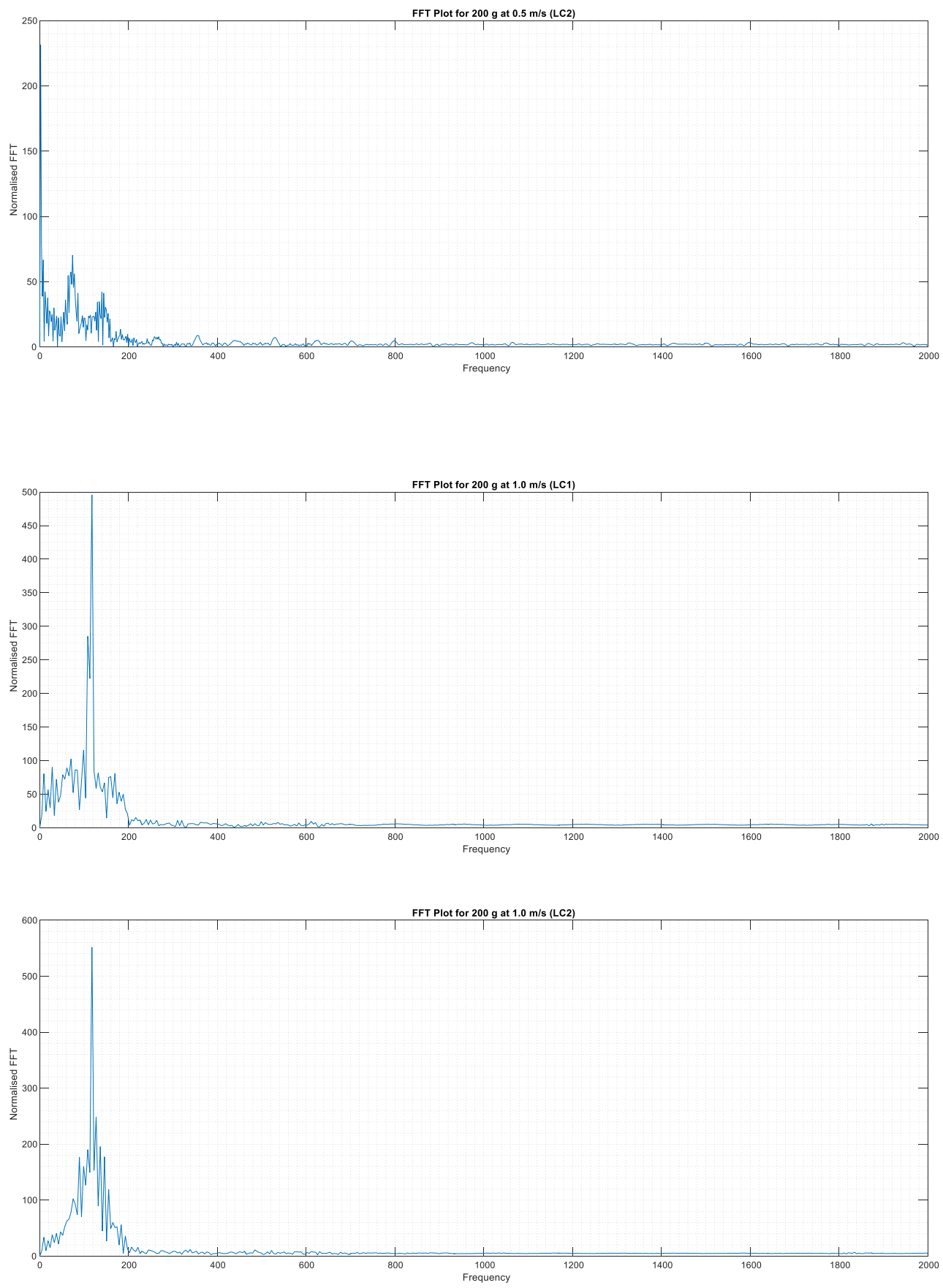

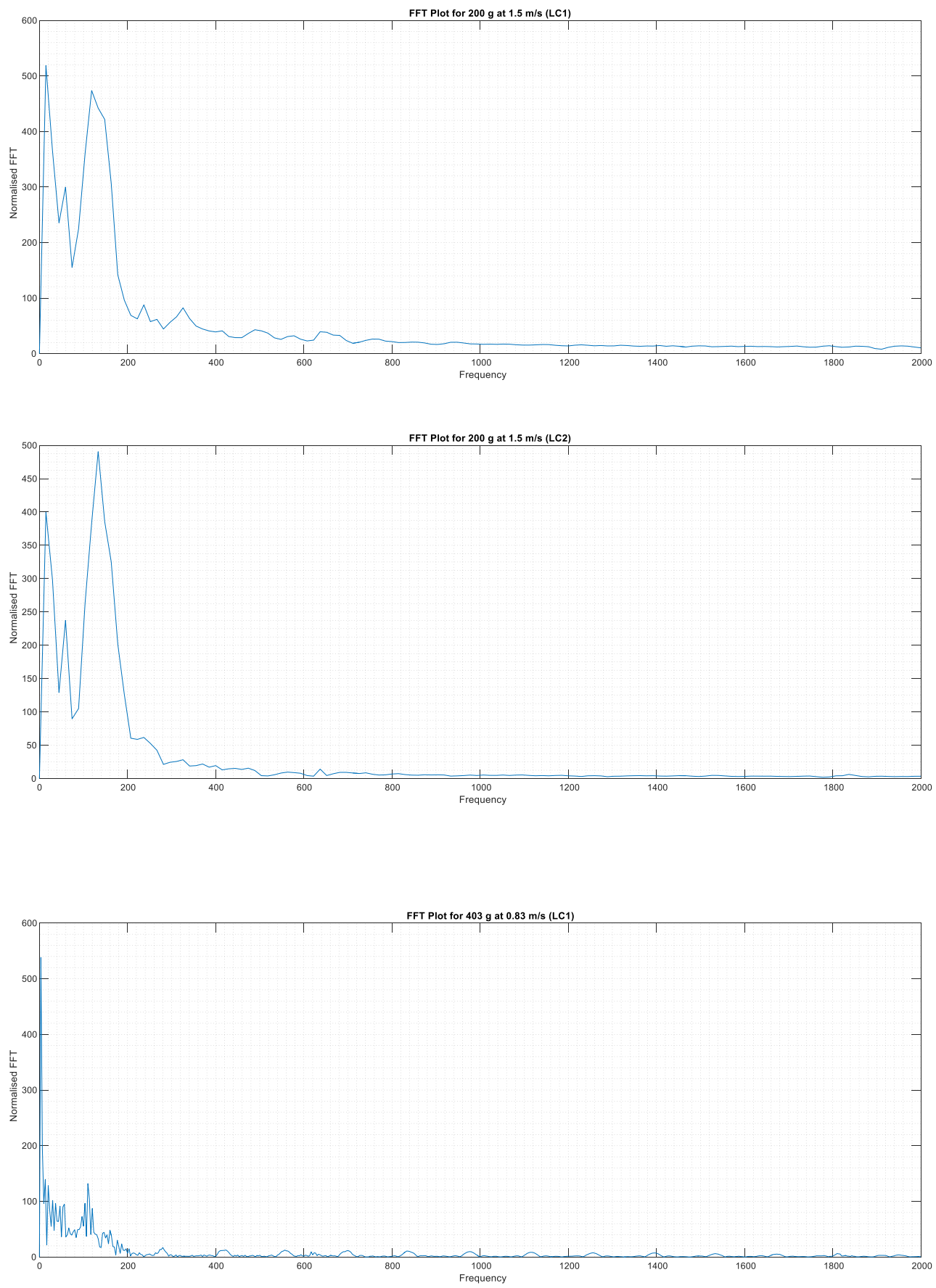

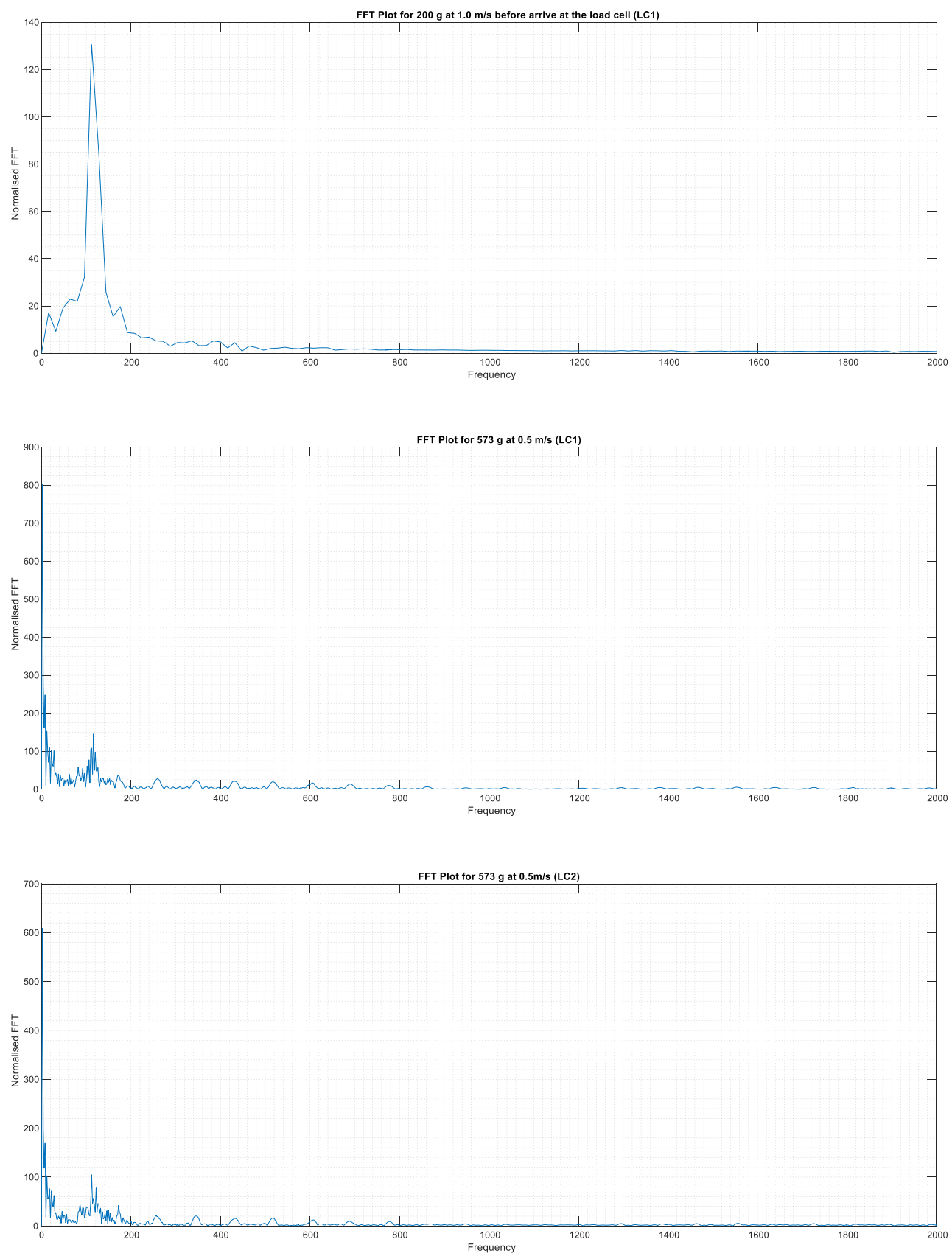

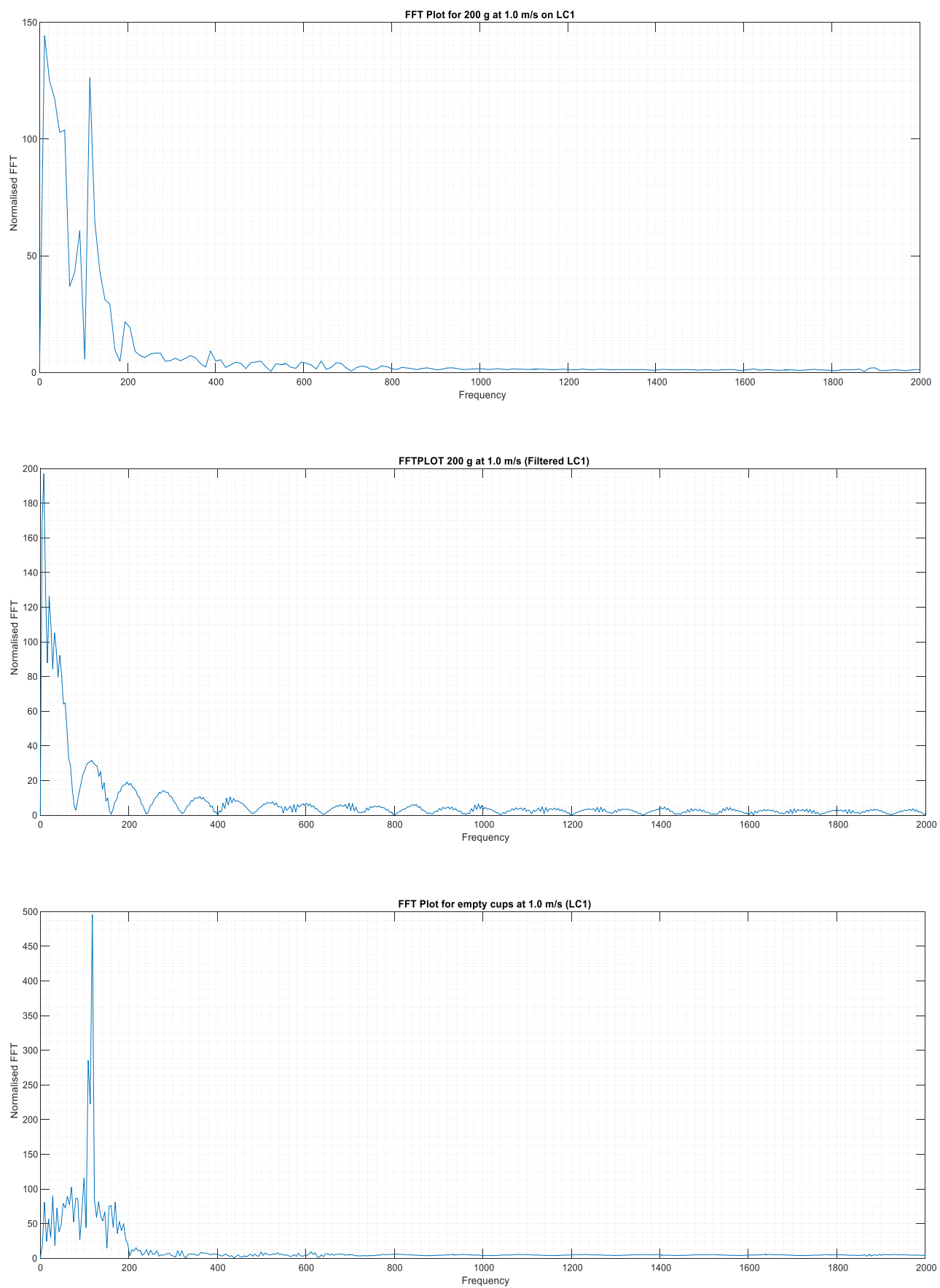


\section{References}

Bateson, R (2002). Introduction to control systems technology, Prentice Hall, New Jersey, United States.

Becker, A. (n.d.). About Kalman filter. Retrieved from kalmanfilter.net.

Bernhard, P \& Deschamps, M (2017). Kalman on dynamics and control Linear System Theory, Optimal Control, and Filter, Working Papers 2017-10, CRESE.

Brignell. J (1991). Software techniques for sensor compensation. Sensors and Actuators A, 25-27 (1991) 29-35.

Calpe, J, Soria, E, Martinez, M, Francis J, Rosado, A \& Vila, J (2002). High speed weighing system based on DSP, IEEE 0-7803-7474-6-02.

Chander, B. (2015, November 3). Does the process noise (Q) and measurement noise (R) keep updating in every iteration while running Extended Kalman Filter at every time step? Message posted to http:// www.reaserchgate.net

Cheever, E. (n,d) Kalman filter to estimate a constant retrieved from http://www.swarthmore.edu/NatSci/echeeve1/Ref/Kalman/Ex1ScalarKalman. html

Doraiswami,R, Diduch,C, Stevenson, M. Identification of Physical systems (John Wiley and sons Ltd, PP 5 -10.

Goldenstein, S (2004). A gentle introduction to predictive filters, RITA Volume 11, https://doi.org/10.22456/2175-2745.5962

Halimic, M \& Balachandran, W. Kalman filter for dynamic weighing system, IEEE Catalogue Number: $95 T H 8081$.

Hardy process solutions. (n.d). Five factors that can affect your weighing system's accuracy, retrieved from https://www.hardysolutions.com/tenants/hardy/documents/5FactorsA.pdf

Harkat, Samra \& Boukharrouba, Malika \& Abdelkader, Douaoui. (2014). Multi-site modeling and prediction of annual and monthly precipitation in the watershed of Cheliff (Algeria). Desalination and water treatment. 12. On line.10.1080/19443994.2014.956798.

Heiberger, M \& and Holland, B. (2004). Statistical analysis and data display. United States of America: Springer science and business media Inc. 
https://crese.univcomte.fr/uploads/documents/f773e79d0a66b93013bdd340f9 83f3aa.pdf

Ifeachor, E \& Jervis, B (1995). Digital signal processing: A practical approach. Addison-Wesley Publishers Ltd. United States.

Jafaripanah, M, Al-Hashimi, B \& White, N (2003). Load cell response correction using analogue adaptive techniques. IEEE proceedings 0-7803-7762-1/03.

Jafaripanah, M, Al-Hashimi, $B$ \& White, N (2005). Application of Analog Adaptive Filters for Dynamic Sensor Compensation. IEEE Transactions on Instrumentation and measurement VOL.54 No.1

Kalman, R (1960). A new approach to linear filtering and prediction problems. Retrieved from

Massachusetts Institute of Technology. (n.d.). Tutorial: The Kalman filter, Lacey, T. retrieved from http://web.mit.edu/kirtley/kirtley/binlustuff/literature/control/Kalman\%20filte r.pdf

McGuinness, M, Jenkins, D \& Senaratne, G, (2005). Modelling the physics of High speed weighing.

Meller, M, Niedzwiecki, M, Pietrzak, P (2014). Adaptive filtering approach to dynamic weighing: a checkweigher case study. IFAC Proceedings Volume 47, Issue 3,2014; PP 5927-5932, http://doi.org/10.3182/20140824-6-ZA1003.00395 .

Mulquiney, J E, J P Norton, A J Jakeman, and J A Taylor. "Random Walks in the Kalman Filter: Implications for Greenhouse Gas Flux Deductions." Environmetrics 6.5 (1995): 473-78. Web.

Niedzwiecki, M, Pietrzak, P. (2017). High-Precision FIR-Model-Based Dynamic Weighing system, .IEEE Transactions on Instrumentation and measurement.

Podesta, J. (1994). Brief tutorial on the Kalman filter. Retrieved from https://apps.dtic.mil/dtic/tr/fulltext/u2/a286571.pdf

Shl, W, White, N \& Brignell, E (1993). Adaptive filters in load cell response correction. Sensors and Actuators A, 37-38 (1993) 280-285.

Shu, W (1993), Dynamic Weighing under Nonzero Initial Conditions, IEEE Transactions on Instrumentation and measurement, Vol. 42, NO. 4. 
Sircar, P. (2016). Paradigm of Signal Processing. In Mathematical Aspects of Signal Processing (pp.1-21). Cambridge: Cambridge University Press. doi:10.1017/9781316796832.003

Thaeh, C. (2018). An Intuitive explanation of expected value. Retrieved from https://www.probabilisticworld.com/intuitive-explanation-expected-value Transactions of the ASME-Journal of Basic Engineering, 82 (Series D): 3545 , retrieved from https://www.cs.unc.edu/ welch/kalman/media/pdf/Kalman1960.pdf

University of Tartu. (n.d.). The concept of measurement, Leito, I. Retrieved from https://sisu.ut.ee/measurement/introduction-concept-measurementuncertainty)

Vernier software and technology (n.d.). What is the uncertainty of my sensor, Retrieved from https://www.vernier.com/til/2294/

Wells, C. (1996). The Kalman filter, Hughes, A (Eds.) The Kalman filter in finance (pp. 75-86). Netherlands: Kluwer academic publishers.

Wilson, D (2016). Advance control using MATLAB, Auckland University of Technology, New Zealand. 